University of Louisville

ThinkIR: The University of Louisville's Institutional Repository

$5-2021$

\title{
The importance of noncapped viral genomic RNA to alphaviral infection and pathogenesis.
}

\author{
Autumn LaPointe \\ University of Louisville
}

Follow this and additional works at: https://ir.library.louisville.edu/etd

Part of the Medical Immunology Commons, and the Medical Microbiology Commons

\section{Recommended Citation}

LaPointe, Autumn, "The importance of noncapped viral genomic RNA to alphaviral infection and pathogenesis." (2021). Electronic Theses and Dissertations. Paper 3594.

https://doi.org/10.18297/etd/3594

This Doctoral Dissertation is brought to you for free and open access by ThinkIR: The University of Louisville's Institutional Repository. It has been accepted for inclusion in Electronic Theses and Dissertations by an authorized administrator of ThinkIR: The University of Louisville's Institutional Repository. This title appears here courtesy of the author, who has retained all other copyrights. For more information, please contact thinkir@louisville.edu. 


\title{
THE IMPORTANCE OF NONCAPPED VIRAL GENOMIC RNA TO ALPHAVIRAL INFECTION AND PATHOGENESIS
}

\author{
By \\ Autumn LaPointe \\ B.S. University of North Carolina Asheville, 2016 \\ M.S. University of Louisville, 2018
}

\begin{abstract}
A Dissertation
Submitted to the Faculty of the

School of Medicine of the University of Louisville

in Partial Fulfillment of the Requirements

for the Degree of
\end{abstract}

Doctor of Philosophy in Microbiology and Immunology

Department of Microbiology and Immunology

University of Louisville

Louisville, Kentucky

May 2021 

THE IMPORTANCE OF NONCAPPED VIRAL GENOMIC RNA TO ALPHAVIRAL INFECTION AND PATHOGENESIS

\section{By}

Autumn LaPointe

B.S. University of North Carolina Asheville, 2016

M.S. University of Louisville, 2018

A Dissertation Approved on

March 25 $5^{\text {th }}, 2021$

by the following Dissertation Committee:

\begin{tabular}{c}
\hline Dr. Kevin Sokoloski \\
\hline Dr. Donghoon Chung \\
\hline Dr. Carolyn Casella \\
\hline Dr. Barbara Clark \\
\hline Dr. Igor Lukashevich
\end{tabular}




\section{DEDICATION}

This dissertation is dedicated to my husband Jordan, who has encouraged me every step of the way, and to my parents who have always supported me in all of my endeavors. 


\section{ACKNOWLEDGEMENTS}

I would first like to thank Dr. Kevin Sokoloski who decided to take a chance on me as his first graduate student. It has been a learning experience for both of us and one for which I am very grateful. Thank you for encouraging me and giving me the confidence and training to stand on my own as a scientist. I consider myself incredibly blessed to have had you as my mentor.

I would like to thank my committee members: Drs. Chung, Lukashevich, Casella, and Clark for their insight and guidance.

I would like to thank my lab mates Claire and Trey, who have made the whole Ph.D journey an enjoyable one. Our lab shenanigans always brightened my day and I could not have asked for better friends. Thank you both for always being willing to listen and/or distract when needed.

I would like to thank Will, Sarah, and Drew for keeping me sane during this endeavor and always being up for a well needed laugh. I would also like to thank my church family, who have been there for me during the good and the bad.

I would like to thank my husband Jordan, who has been my biggest source of encouragement. Thank you for constantly supporting me and reminding me of what I am capable of. I could not have done it without you and am so very thankful that you came on this adventure with me.

Finally, I would like to thank my family, who have always been supportive of me and my love of science, which is what inspired me to obtain my Ph.D. to begin with. Thank you for believing in me and teaching me that I could accomplish whatever I put my mind to. 


\title{
ABSTRACT \\ THE IMPORTANCE OF NONCAPPED VIRAL GENOMIC RNA TO ALPHAVIRAL INFECTION AND PATHOGENESIS
}

Autumn LaPointe

March 25, 2021

\begin{abstract}
Alphaviruses are mosquito-borne arboviruses that have a positive sense, single-stranded RNA genome, which are capable of causing severe disease and thus pose a significant burden to public health. During alphaviral replication, significant quantities of viral genomic RNAs that lack the canonical alphaviral 5' cap structure are produced and packaged into viral particles, despite the fact that these noncapped genomes cannot be translated and are essentially noninfectious. The production and packaging of the noncapped genomes has been found to be true for infections across multiple vertebrate and invertebrate cell lines and alphavirus species, although the proportion of ncgRNA produced differs. Despite knowing this, the importance of the noncapped genomic vRNA (ncgRNA) to alphaviral infection has yet to be fully characterized. In this dissertation, the importance of the ncgRNAs to alphaviral infection was assessed by using mutations of the nsP1 protein of Sindbis virus (SINV), which altered the synthesis of the ncgRNAs during infection by modulating the protein's capping efficiency. It was found that decreasing ncgRNA production lead to decreased
\end{abstract}


viral growth kinetics via decreased particle production; whereas increasing ncgRNA production led to wild type growth kinetics and particle production.

In addition to being necessary for efficient viral replication in vitro, we also found that the noncapped genomic RNA was critical for alphaviral pathogenesis. Mice infected with the increased ncgRNA mutant exhibited wild type rates weight loss, and neurological symptoms, but exhibited a slight decrease in mortality. Conversely, the mice infected with the decreased ncgRNA mutant showed significantly reduced mortality and morbidity compared to mice infected with wild type virus. Interestingly, both mutants had modestly reduced viral titer in the brain compared to wild type virus. Importantly, examination of the brain tissue revealed that mice infected with the decreased ncgRNA mutant had significantly reduced cell death, immune cell infiltration, and expression of proinflammatory cytokines compared to the increased ncgRNA mutant and wild type virus.

Collectively, these data indicate that the ncgRNA play an important role during alphaviral replication and pathogenesis by increasing the efficiency of particle production and modulating the host immune response. 


\section{TABLE OF CONTENTS}

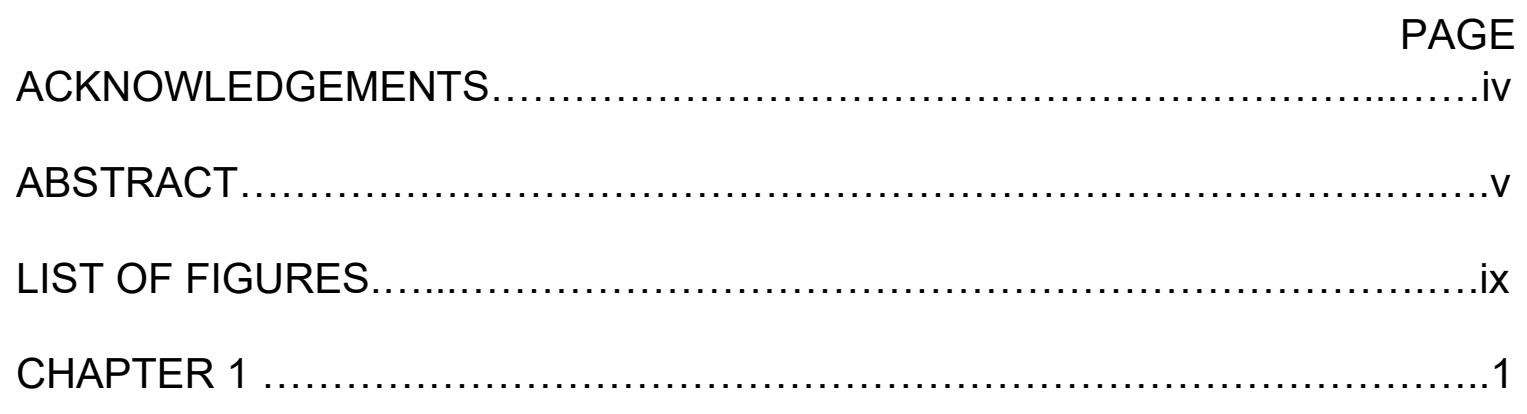

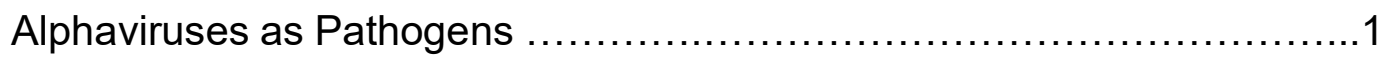

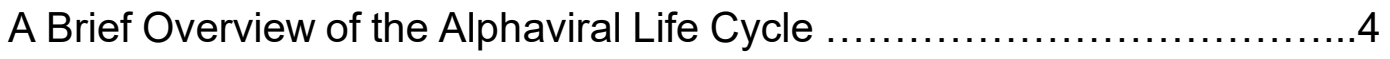

Alphavirus Genetic Organization . ................................. 5

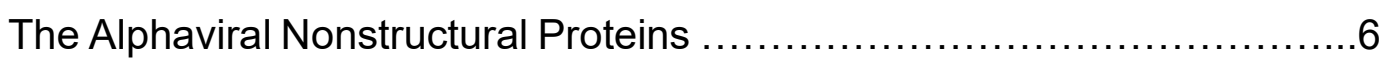

The Alphaviral Structural Proteins ..................................12

Alphaviral Arthritis in Mouse Models of Infection .......................15

Alphaviral Encephalitis in Mouse Models of SINV Infection ...............17

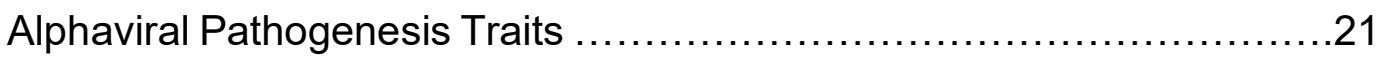

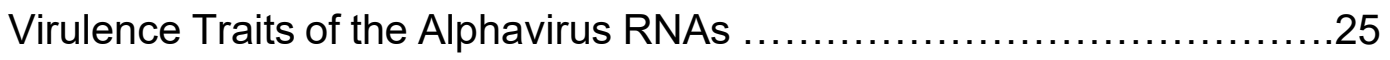

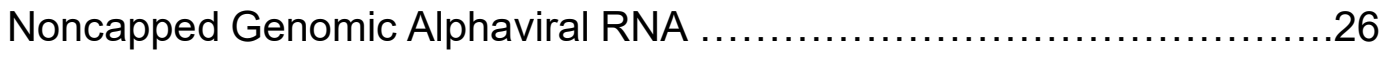

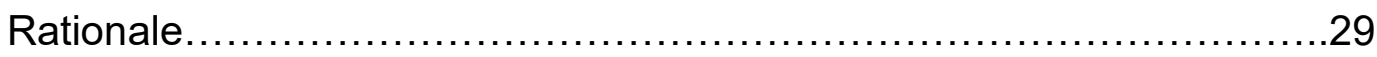

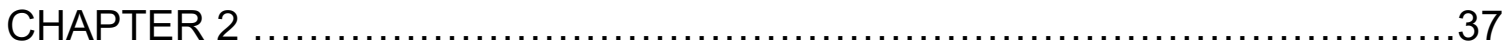

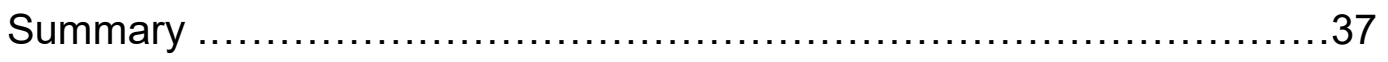

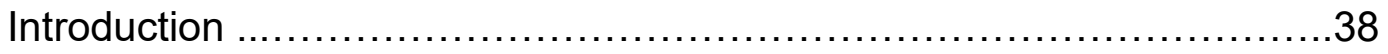

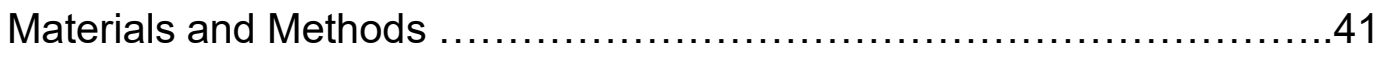


Results

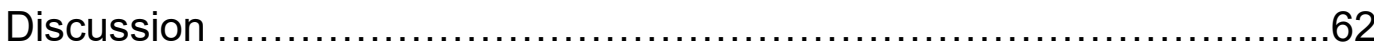

Figures \& Figure Legends..............................................

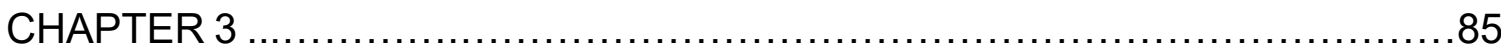

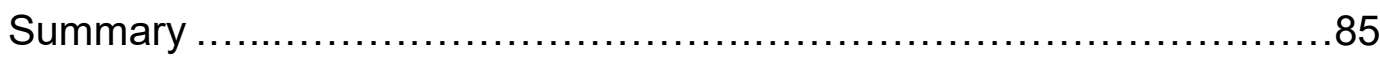

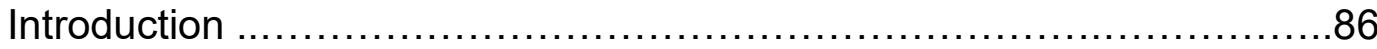

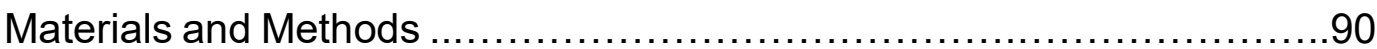

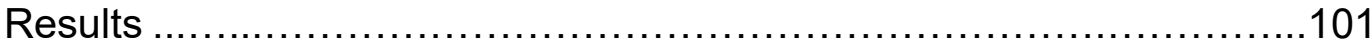

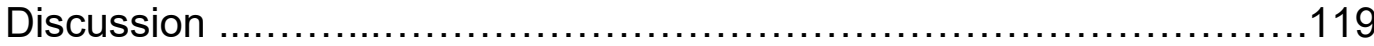

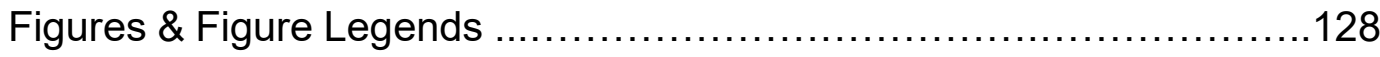

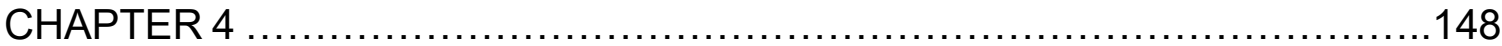

Research Summary .................................................. 148

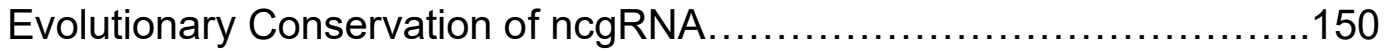

Role of the ncgRNA in Particle Production................................153

Role of ncgRNA in Pathogenesis......................................156

ncgRNA as a Novel Antiviral Target....................................157

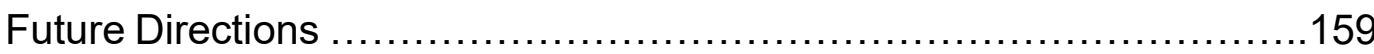

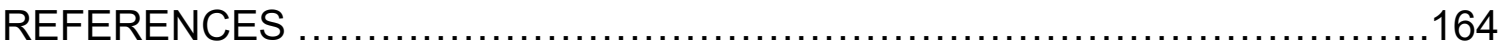

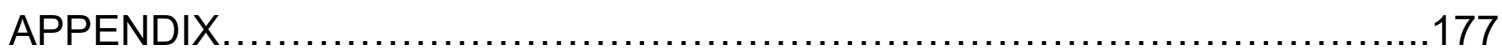

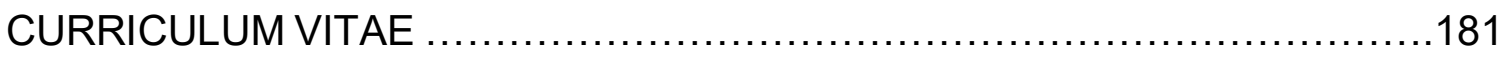




\section{LIST OF FIGURES}

\section{PAGE}

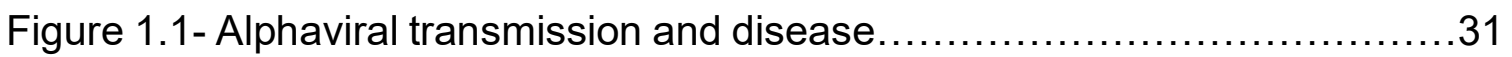

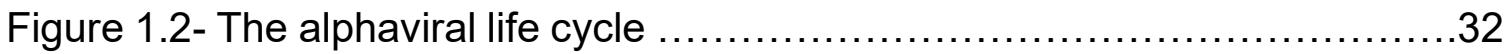

Figure 1.3- Alphaviral RNA replication and genetic organization ................33

Figure 1.4- Capped and noncapped genomic RNA are produced during alphaviral

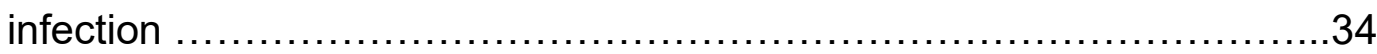

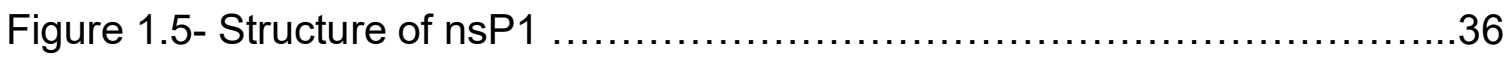

Figure 2.1- Point mutations in the nsP1 protein of SINV alter 5' vRNA capping efficiency. .71

Figure 2.2- Altering viral capping efficiency negatively impacts viral infection....73

Figure 2.3- Translation of the genomic vRNA correlates with viral capping

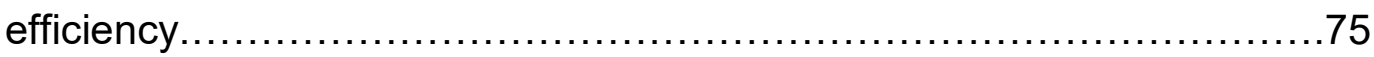

Figure 2.4- nsP2 protein levels are impacted by mutation of the nsP1 protein...77

Figure 2.5- Altering vRNA capping efficiency impacts early RNA synthesis.....80

Figure 2.6- Subgenomic gene expression is unaffected by altering SINV vRNA

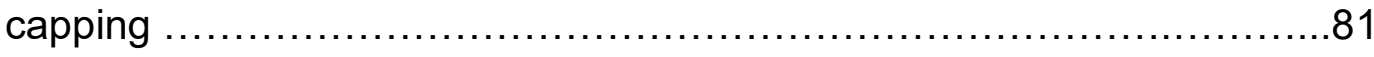

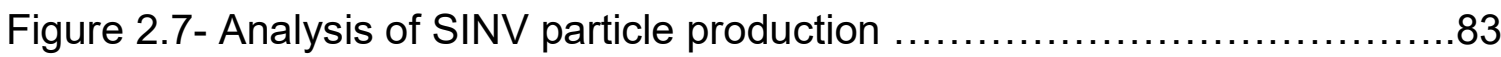

Figure 2.8- Proposed model of how increasing genomic vRNA capping negatively

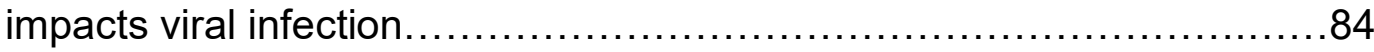

Figure 3.1- Point mutations in nsP1 of AR86 SINV result in changes in capping efficiency and negatively impacts infection in mammalian cells 128 
Figure 3.2- Increasing capping efficiency increases translation of SINV AR86 nonstructural polyprotein.

Figure 3.3- Altering capping efficiency does not impact AR86 SINV vRNA synthesis

Figure 3.4- Production of type-I interferon and ISGs in response to SINV nsP1 capping mutants

Figure 3.5- Analysis of SINV sensitivity to type-I interferon. 135

Figure 3.6- Increased vRNA capping efficiency reduces SINV AR86 mortality and pathogenesis. 136

Figure 3.7- Increased capping efficiency leads to decreased pathology in the brain. 138

Figure 3.8- Viral replication is largely unaffected by altered capping in vivo ....139

Figure 3.9- Neuron viability increased with decreased capping efficiency......140

Figure 3.10- Increased viral capping efficiency results in reduced expression of pro-inflammatory genes in the brain

Figure 3.11. Proposed model of how increasing genomic vRNA capping negatively impacts viral pathogenesis 145

Supplemental Figure 3.1- Quantitative analysis of IFN $\beta$ transcript levels in SINV AR86 infected ankle tissue. 146

Figure 4.1- Potential roles for ncgRNA in alphaviral particle production. 162 


\section{CHAPTER 1}

\section{INTRODUCTION}

\section{Alphaviruses as Pathogens}

Alphaviruses are mosquito-borne, single-stranded RNA viruses that have been the cause of both localized outbreaks and large epidemics of severe disease. Transmission of these viruses largely occurs between a mosquito vector, primarily Aedes, Anopheles, and Culex genera, and a vertebrate host, typically rodents and birds, in an enzootic cycle (Fig. 1.1) [1]. However, epizootic spillover events do occur that lead to infection of humans and horses. Although these tend to be dead-end hosts, recent alphavirus epidemics have shown the ability of these viruses to adapt for more efficient interhuman transmission [2]. Due to the expansion of the geographic area in which the mosquito vectors can be found, alphaviruses have an increased capacity to emerge into naïve populations, making them a significant threat to public health.

Alphaviruses are largely classified as either arthritogenic or encephalitic based on the symptoms of infection. Arthritogenic alphaviral infection causes disease that can vary from mild to severe and can persist for several months to years past the acute phase of infection [3]. This includes Chikungunya (CHIKV) and Ross River Virus (RRV) which are capable of causing debilitating polyarthritis as well as the model alphavirus Sindbis virus (SINV), which is the 
causative agent of rash-arthritic diseases like Pogosta disease, Ockelbo disease, and Karelian fever [4-8]. While not associated with high rates of mortality, the high morbidity of arthritogenic alphaviral disease results in a high economic burden that is particularly damaging in regions where labor-intensive work is prevalent $[9,10]$. The encephalitic alphaviruses include Venezuelan, Eastern, and Western Equine Encephalitis viruses (VEEV, EEEV, and WEEV) and are capable of causing severe meningitis and encephalitis, as well as long lasting sequelae such as seizures, paralysis, and cognitive deficits in survivors [11-14]. These viruses, while comparatively rare in regards to their incidence, typically have high mortality rates, especially in comparison to the arthritogenic alphaviruses, with viruses like EEEV having mortality rates as high as $70 \%$ in symptomatic individuals [15].

As part of their transmission cycles, alphaviruses infect both mosquitos as well as vertebrates. Infection of the mosquito vector begins with the ingestion of a bloodmeal from an infected vertebrate host. Viral infection is initiated in the midgut, where the virus infects and replicates within epithelial cells lining the gut $[16,17]$. Once the virus has escaped from the midgut, it then spreads to the hemocoel, an open body cavity where hemolymph is circulated. Once in the hemolymph, the virus is spread to secondary tissues, including the salivary glands, which is the main site of viral transmission [16]. Once the salivary glands have been infected, the virus can be released into the salivary ducts and transmitted orally to a vertebrate host during the mosquito's next blood meal. The extrinsic incubation period, which is the time from ingestion of the infected 
bloodmeal to when the mosquito is able to transmit the virus, is $\sim 2-7$ days for alphaviruses [1]. While the mosquito immune system is able to limit viral replication, it is unable to completely clear the virus, thus allowing the virus to be continuously transmitted to new vertebrate hosts for the life of the mosquito [18]. After being transmitted to a vertebrate host, the virus replicates locally at the site of inoculation in the skin, specifically dermal fibroblasts, enterocytes, and keratinocytes [19]. Infection of Langerhan's cells and dendritic cells in the skin leads to infection of the lymph nodes draining the site of inoculation. Once viremia occurs, the virus is then able to disseminate to other tissues, including lymphoid tissues, joints, and the central nervous system including the brain [1].

Which secondary organs the virus disseminates to is largely dependent on the particular alphavirus species, as tissue tropism differs greatly among the alphaviruses. The arthritogenic alphaviruses primarily target the cells of the skin, joints, and muscle, while the encephalitic alphaviruses target those of the nervous system [1]. Inflammation in these target regions in response to viral infection then ultimately leads to the development of alphaviral disease. The time from infection to onset of symptoms, termed the intrinsic incubation period, ranges from 2-10 days in people for both the arthritic and encephalitic alphaviruses $[20,21]$.

Despite the threat that alphaviruses pose to public health, there are no antiviral strategies or vaccines which are either safe or effective for preventing alphaviral infection or treating alphaviral disease. This deficit of viable therapeutics highlights the need to better understand the mechanisms behind 
alphaviral infection in order to develop novel antiviral strategies for the mitigation of alphaviral disease.

\section{A Brief Overview of the Alphaviral Life Cycle}

Infection begins with the E2 glycoproteins in the viral envelope binding to receptors on the surface of the host cell (Fig. 1.2). After binding, the virus rapidly enters the host cell via receptor mediated endocytosis, which forms an endosome containing the viral particle $[22,23]$. As the endosome transitions from early to late, the low $\mathrm{pH}$ of the maturing endosome causes conformational changes in the glycoproteins which allows the envelope to fuse with the endosomal membrane, releasing the viral nucleocapsid into the cell cytoplasm [24]. Membrane fusion is mediated by the viral E1 glycoprotein after the exposure of the fusion loop during the conformational change in response to $\mathrm{pH}$ [25]. Following the release of the nucleocapsid core, the host 60 S ribosomal RNA interacts with the capsid protein and facilitates the disassembly of the nucleocapsid, resulting in the viral genome being released into the cytoplasm [26]. The genome then interacts with host factors and is immediately translated by host cell machinery, producing the nonstructural polyprotein P1234, which is then proteolytically cleaved via the proteolytic activities of nonstructural protein 2 (nsP2) to form the initial viral replicase complex, P123 and nsP4. This initial complex uses the viral genome to synthesize the minus strand RNA that serves as the template for viral replication and transcription [27]. The nonstructural polyprotein is then further processed by nsP2 to produce the individualized 
nonstructural proteins of the fully processed viral replicase complex (consisting of nsP1, nsP2, nsP3, and nsP4) [28]. The replicase complex produces the genomic RNA and subgenomic RNA through viral replication and transcription, respectively. The subgenomic RNA is translated to produce the structural polyprotein, consisting of the capsid protein, the glycoproteins E3; E2; and E1, and the $6 \mathrm{~K} /$ transframe (TF) proteins. The capsid protein cleaves itself off of the structural polyprotein and associates with the newly synthesized viral genomes to form nucleocapsid cores [29]. The remaining structural polyprotein is targeted to the endoplasmic reticulum via signal sequences present in the E3 protein, and subsequently processed into the individual glycoproteins in the ER via the activities of host proteases, where they undergo post-translational modifications

including disulfide bond rearrangement, palmitoylation, and glycosylation prior to being trafficked to the cell surface [30]. Newly formed mature virions are then released from the cell via budding, creating the envelope that contains the viral glycoproteins as the nucleocapsid core pushes through the host plasma membrane [30].

\section{Alphavirus Genetic Organization}

The alphaviral genome is a single-strand of positive sense RNA that is $\sim 11.5 \mathrm{~kb}$ in length and contains two coding regions. The first open reading frame is the only one expressed from the genome and codes for the nonstructural proteins nsP1-4, which are responsible for RNA replication (Fig. 1.3). The nonstructural proteins are expressed as a polyprotein, P1234, that undergoes 
several iterations of proteolytic cleavage to form the various replication complexes needed to produce the minus strand template and subgenome in addition to more genomic RNA. The minus strand RNA, which serves as the template from which the genome and subgenome, is the predominant vRNA synthesized by a complex consisting of $\mathrm{P} 123$ and nsP4. Once the viral polyprotein has been fully cleaved into $\mathrm{nsP} 1,2,3$, and 4 , they form the replication complex that synthesizes the genome and subgenome. The subgenome contains only the second open reading frame and is responsible for the production of the structural proteins. The structural proteins include the capsid protein, glycoproteins, and the $6 \mathrm{k} / \mathrm{TF}$ protein, which are all required to form the viral particle.

\section{The Alphaviral Nonstructural Proteins}

As stated earlier, there are four nonstructural proteins (nsPs) encoded as a single polyprotein during the translation of the viral genomic RNA. These are named numerically in the order of their relative position to the $\mathrm{N}$-terminus of the polyprotein. Each of the individual nsPs are necessary for viral replication with their primary functions in viral replication being modular in nature; and in addition to their viral RNA synthesis roles, several of the nsPs have critical functions outside of viral replication complex.

\section{The Alphaviral nsP1 Protein}


The $\sim 60 \mathrm{kDa}$ nsP1 protein serves three primary functions during alphaviral infection: anchoring the replication complex, forming the pore to the replication spherules, and vRNA capping via methyltransferase and guanylyltransferase enzymatic reactions [28]. The presence of an amphipathic helix, and the palmitoylation of conserved cysteine residues in nsP1, allows the protein to anchor the replication complexes to host membranes, which is a consistent feature of the replication machinery of all ssRNA viruses. Binding of nsP1 to the host membrane starts with weak binding of the amphipathic helix to plasma membrane. This is followed by palmitoylation of cysteine residues within the amphipathic helix, which changes the properties of nsP1 to appear similar to those of the integral membrane proteins [31]. The interaction of the nsP1 protein with host membranes is associated with the formation of subcellular structures important to alphaviral biology, including the formation of membranous spherules, and the formation of filopodia at the cell surface which aid in cell-tocell transmission [31, 32]. The spherules are formed from host membranes, with the specific host membrane varying across the members of the genus, and serve as the site of RNA replication. In addition to anchoring the replication complex and lining the spherule compartment, the nsP1 protein has also been shown to form the pore structures that are in part responsible for controlling access to the replication spherules [33]. In a recent publication from Jones et al that determined the structure of nsP1, they demonstrated that the nsP1 proteins assemble into a dodecameric ring structure that would allow the protein to restrict access to the replication spherule. Whether the guanylytransferase or 
methyltransferase activities of nsP1 are active while in this pore-like structure is unknown, but the constriction of the pore is important for keeping the dsRNA intermediates that form during RNA replication from being sensed by host proteins like MDA5, LGP2, and Dicer [34]. While the membrane binding properties of the nsP1 protein are dispensable to viral replication, the efficiency of RNA synthesis in mutants lacking membrane association is greatly impaired. Nonetheless, nsP1's role as a membrane binding protein is separate from its function as the capping enzyme, as binding to the host membrane does not impact the enzymatic activity of nsP1 [35-37].

The nsP1 protein's role as the alphaviral capping enzyme has been fairly well characterized. Both the genome and subgenome have a Type-0 cap structure co-transcriptionally added to the 5' end during RNA synthesis. The Type- 0 cap used by alphaviruses is similar to the Type- 1 cap added to eukaryotic mRNAs, with the major difference being that the Type-1 cap is $2^{\prime} O$ methylated whereas the Type-0 cap is not [38]. The addition of the cap to the 5' end of the viral RNA serves multiple critical functions in virus replication as well as how the vRNA interacts with the host cell. The presence of the 5' cap structure allows the genome and subgenome to be readily translated using the host translation machinery. In addition, the 5' cap also protects the RNA from being degraded by host exonucleases and prevents immune sensing of the viral RNA by host proteins such as RIG-I.

The addition of the cap to the 5' end of the vRNA is carried out in a stepwise fashion by nsP1 and nsP2. The methyltransferase domain of nsP1 
transfers a methyl group from S-adenosylmethionine (SAM) to the N7 position of a GTP molecule. The guanylyltransferase region of nsP1 then binds the m7Gppp nucleotide forming a covalent link with the catalytic histidine residue (specifically $\mathrm{H} 39$ in SINV), releasing a molecule of inorganic pyrophosphate (PPi) and resulting in the formation of an m7GMP-nsP1 intermediate $[35,39]$. By this time the nsP2 protein has removed the $5^{\prime} \mathrm{Y}$-phosphate from the nascent $\mathrm{VRNA}$, and nsP1 transfers the m7GMP moiety to the vRNA molecule, resulting in the formation of the Type-0 7meGppA cap structure [40]. The alphaviral RNA capping mechanism is considered to be unconventional, as the methylation of the GTP and the guanylyltransferase activities are in the reverse order compared to how RNA capping occurs in eukaryotic cells, where the guanine moiety is added to the RNA first and then methylated [38]. Importantly, and as described in greater depth below, the capping activities of the nsP1 protein can be modulated via the incorporation of point mutations.

\section{The Alphaviral nsP2 Protein}

The $\sim 90 \mathrm{kDa}$ nsP2 protein has several distinct domains and has a diverse range of functions during the viral lifecycle [28]. The protease domain of nsP2 is responsible for cleaving the nonstructural polyprotein in cis- and in trans- during viral RNA synthesis, specifically through the processing of P1234 into $\mathrm{P} 123+n s \mathrm{P} 4$ via cis-cleavage, and then further cleaving P123 into nsP1+P23, and later $\mathrm{P} 23$ into $\mathrm{nsP} 2$ and nsP3 via trans-cleavage events [41]. Viruses lacking this proteolytic activity have been shown to be unable to complete the viral lifecycle 
as the maturation of the RNA synthetic complex is essential to viral RNA synthesis [42]. Additionally, in regards to viral replication and transcription the nsP2 protein functions as an RNA helicase and an RNA triphosphatase. The helicase activity of nsP2 allows it to unwind RNA secondary structures during viral replication [43]. As mentioned above, the RNA triphosphatase motif in nsP2 allows it to remove a phosphate from the 5' nucleotide, which prepares the vRNA for subsequent capping. In addition to the helicase and triphosphatase domains the nsP2 protein has a methyltransferase domain that is presumed to be enzymatically inactive as it lacks the catalytic residues of known methyltransferase enzymes [44]. The precise function / importance of this domain is unknown at this time.

In addition to the roles described above, the nsP2 protein of Old World alphaviruses (including SINV) is intimately involved in the shutoff of host transcription during viral infection [45]. The shutoff of host transcription, and its importance to the evasion of the host antiviral defenses are described in greater detail below.

\section{The Alphaviral nsP3 Protein}

Compared to the other alphaviral nonstructural proteins the alphaviral $\mathrm{nsP} 3$ protein has been enigmatic, while necessary for RNA replication, the exact roles that nsP3 plays in this process are poorly defined. Structurally the $\sim 60 \mathrm{kDa}$ nsP3 protein consists of three distinct regions- an $\mathrm{N}$-terminal Macrodomain, an Alphavirus Unique Domain (AUD), and a C-terminal hypervariable region that is 
multiply phosphorylated during infection [28]. The N-terminal Macrodomain exhibits the highest level of sequence conservation across the members of the genus, and is known to have ADP-ribosylase activity, which has been correlated with its ability to disassemble and prevent stress granule formation during viral replication $[46,47]$. The importance of ADP-ribosylation is an area of current study. Similarly, the AUD is not well understood, but mutational analyses of the domain have indicated important roles in viral RNA synthesis, particularly in regards to the synthesis of the subgenomic vRNAs [48]. The C-terminal hypervariable region includes binding motifs for many host proteins and is thought to act as a scaffold which may be important for the assembly of the replication complex [49]. Interestingly, the hypervariable region exhibits remarkable diversity both at the amino acid level and the identities of the specific interacting host factors.

\section{The Alphaviral nsP4 Protein}

The $\sim 70 \mathrm{kDa}$ alphaviral nsP4 protein is the viral RNA dependent RNA polymerase (RdRp) [28]. Many alphaviruses produce less nsP4 than the other nonstructural proteins, primarily due to the presence of a leaky Opal stop codon at the end of nsP3 which will occasionally terminate translation before nsP4 has been synthesized [50]. The nsP4 protein is solely responsible for carrying out RNA synthesis; however, nsP4 cannot act on its own, and can only function properly in association with the other nonstructural proteins as the replicase complex [51]. Structurally the nsP4 protein is largely unremarkable when 
compared to the RdRps of other positive-sense RNA viruses. Regardless, there are nuances specific to the alphaviral RdRp, including the absolute requirement of an $\mathrm{N}$-terminal tyrosine residue for activity, the function of which is unclear at this time. Additionally, the alphaviral nsP4 protein is responsible for the synthesis of the poly $(A)$ tail found on the positives-sense vRNAs [28]. It should be noted that the nsP4 protein lacks any discernable proof-reading capacity, resulting in a relatively high rate of mutation and the formation of a diverse quasispecies [52].

\section{The Alphaviral Structural Proteins}

As with the nonstructural proteins, the synthesis of the structural proteins begins with the synthesis of a polyprotein from the subgenomic vRNA from which the individual proteins are generated via proteolytic processing events. While the subgenomic RNA is known to be capped it is believed to utilize a noncanonical cap-independent translation initiation mechanism [53]. This is due to the cessation of cap-dependent translation during alphaviral infection following the activation of the host PKR response leading to phosphorylation of elF2 $\alpha$ in response to the synthesis of viral dsRNA. Regardless, the translation of the subgenomic vRNA leads to the production of 6 structural proteins- capsid, the E3; E2; and E1 glycoproteins, the 6k, and transframe (TF) proteins.

\section{The Alphaviral Capsid Protein}

The $\sim 30 \mathrm{kDa}$ alphaviral capsid protein lacks a discernable beta-sheet jelly roll conformation, and thus, is structurally unique compared to capsids of other 
positive-sense RNA viruses [54, 55]. Architecturally the alphaviral capsid protein may be broadly divided into two domains- an $\mathrm{N}$-terminal polybasic region with little structure, and a well conserved C-terminal protease domain. The $\mathrm{N}$-terminal region lacks mentionable sequence conservation outside of being generally positively charged and being relatively proline rich. Subdomains exist within the $\mathrm{N}$-terminal domain, with specific regions having known functional roles during the alphaviral lifecycle. The $\mathrm{N}$-terminal subdomain is believed to mitigate the negative charge of the vRNA in the context of the nucleocapsid [56]. Immediately adjacent to the RNA-binding domain is an alpha helix known to be involved in the formation of capsid dimers during the viral assembly process. Finally, the third subdomain exhibits a moderate degree of sequence conservation and is involved in the selection of the viral genomic RNA during packaging as well as the release of the genomic RNA from the nucleocapsid upon the binding of the 60S rRNA $[56,57]$.

In contrast to the $\mathrm{N}$-terminal domain, the C-terminal Protease domain is rather well conserved amongst the members of the genus. The proteolytic activity of the protease domain is responsible for the initial cleavage of the capsid protein from the structural polyprotein, after which the C-terminal tail of the capsid protein inactivates the protease active site via steric hindrance $[58,59]$.

In addition to the structural roles of the alphaviral capsid protein, the capsid protein of New World alphaviruses are responsible for the shutoff of host gene expression, as described in greater detail below. 


\section{The Alphaviral Glycoproteins}

After the cleavage of the capsid protein from the structural polyprotein encoded by the subgenomic RNA the signal sequence present in the E3 protein guides the synthesis of the remaining proteins into the host ER [60]. As with the nonstructural polyprotein a series of proteolytic cleavage events lead to the production of the individual viral glycoproteins; however, unlike the previous polyprotein processing events, these cleavages utilize host proteases [61, 62]. Initially, the $6 \mathrm{~K}$ and $\mathrm{E} 1$ proteins are liberated from the polyprotein via the activity of signal peptidases, leaving the remaining polyprotein consisting of the uncleaved E3 and E2 proteins. This sub-polyprotein, termed PE2 (or P62), is then processed by host furin protease to form the final glycoproteins [62]. During this time the viral E2 and E1 glycoproteins are engaged with one another as a heterodimer, and the cleavage of E3 from E2 serves to prime the receptor complex for future entry events [63]. Also during this time, the viral glycoproteins

are post-translationally modified as they traffic from the ER to the Golgi Complex, and ultimately, to the cell surface where they will interact with newly formed nucleocapsid cores leading to the release of mature viral particles.

The $\sim 49 \mathrm{kDa}$ alphaviral E2 protein has three ectodomains, termed A, B, C relative to their position towards the $\mathrm{N}$-terminus, and one subdomain $\mathrm{D}$ in close proximity to domain $C[55,64]$. The functions of these domains have been characterized largely through the use of deletion mutants, or point mutants at the E1/E2 interface. Domain B is the receptor binding domain, and in the context of the glycoprotein heterodimer, domains $A$ and $B$ cover the fusion loop of the $E 1$ 
protein. The $\mathrm{D}$ subdomain interacts extensively with the E3 protein during the maturation process. In addition, the C-terminal tail of the E2 protein interacts with a hydrophobic pocket on the capsid protein leading to agreement between the symmetries of the glycoprotein spikes and the nucleocapsid cores.

The $\sim 52 \mathrm{kDa}$ alphaviral E1 protein has three domains, termed I, II, and III on the basis of their position relative to the $\mathrm{N}$-terminus $[55,65]$. The central domain of E1, domain I, connects to the flanking domains of II and III. Domain II contains the viral fusion loop peptide, which as described above, is obscured by the E2 protein until a conformational trigger in response to low $\mathrm{pH}$ [66]. Domain III is largely involved in the structural rearrangements that lead to the fusion of the virus and host membranes during entry.

In addition to the major glycoproteins described above, the $\sim 6 \mathrm{kDa} 6 \mathrm{~K}$ and $\sim 8 \mathrm{kDa}$ TF proteins are alternatively produced during translation [67]. The $6 \mathrm{k}$ and TF proteins are encoded by the same gene, but which protein is produced is determined by a frameshift event at a slip site upstream of the gene [67]. The roles of these proteins in viral infection have not been well characterized, but they are known to be important for particle assembly and budding [68].

\section{Alphaviral Arthritis in Mouse Models of Infection}

The arthritogenic alphaviruses include Chikungunya, Ross River, Semliki Forest, Burmah Forest, Mayaro, and Sindbis virus. Each of these viruses have been demonstrated to be capable of infecting mice and causing pathogenesis, however the ultimate disease manifestations are dependent on the route of 
inoculation. In order to model arthritic disease in mice, the virus is inoculated subcutaneously in the rear footpad, where it replicates in skin fibroblasts and produces high titer viremia. The virus then spreads to the muscle, skeletal muscle, and joints, resulting in immune infiltration and inflammation in and around the synovial membranes and connective tissue. This leads to the development of acute disease which involves biphasic swelling of the inoculated foot, which peaks around days 3 and 7 post inoculation in adult immunocompetent mice $[69,70]$. While infectious virus is cleared and swelling goes down approximately 2 weeks post inoculation, viral RNA remains present in the joint for at least 16 weeks following infection. Severity and presentation of disease varies with age, as infection in neonatal mice results in lethal encephalitis (Ross, 1956; Couderc, 2009). The change in lethality and disease symptoms between young and adult mice is thought to largely be due to the maturation of the immune system, as the immune system of neonatal mice is not yet matured [71].

Severity of arthritic disease is thought to be largely mediated by the host immune response. This is largely supported by studies with CHIKV and RRV using Rag -/- mice, which are deficient in B-cells and T-cells. In RRV infection, viral replication and progression of disease mirrored what is seen in WT mice, leading to the conclusion that innate immune infiltration and cytokine production are what lead to inflammation in the joint [72]. However, Rag -/- mice infected with CHIKV show little swelling of the foot during infection, suggesting that the adaptive immune response plays a much larger role in induction of inflammation 
in CHIKV compared to RRV [73]. In particular, CD4+ T-cells have been shown to be essential for the development of joint swelling [74]. In addition to T-cells, macrophages in particular have been linked to progression of disease, as mice treated with a drug which inhibits production of MCP-1, a major monocyte chemoattractant, prior to CHIKV infection developed significantly milder disease and exhibited reduced immune infiltration in the muscle and joint [75]. The adaptive immune response is known to play a much larger role in viral clearance. This is supported by a study which showed that Rag -/- mice infected with CHIKV exhibit persistent viremia and elevated viral burdens compared to wild type C57BL/6 mice [73]. B-cells are thought to be especially important for clearance of CHIKV as B-cell deficient mice are unable to clear infection and pre-treatment of IFNAR -/- mice with anti-CHIK antibodies was found to be sufficient to protect against lethality $[76,77]$.

\section{Alphaviral Encephalitis in Mouse Models of SINV Infection}

The encephalitic alphaviruses include Venezuelan, Eastern, and Western Equine Encephalitis Virus. Even though SINV causes arthritis in humans, specific strains such as AR86 cause lethal encephalitis in mice and thus, SINV serves as a model for alphaviral neuropathogenesis in mice. Depending on the aim of the study, the virus is typically inoculated either directly into the brain through intracranial injection or subcutaneously in the rear footpad. During subcutaneous infection, the virus quickly replicates in the muscle near the sight of inoculation and rapidly produces high titer viremia. The virus then targets the central nervous 
system and brain, where it primarily infects neurons in the hippocampus and the anterior horn of the spinal cord, resulting in large scale inflammation and encephalitis [78]. At this stage, mice typically develop severe neurological symptoms, including paralysis of one or multiple limbs, and experience significant weight loss. Death due to encephalitis occurs between 7-9 days post infection for 5-week old C57BL/6 mice [79]. Severity of disease is dependent on age, as fully matured mice do not succumb to disease and do not develop severe neurological symptoms $[79,80]$. This age-dependent resistance to SINV infection is believed to be largely due to the maturation of neurons in the brains and central nervous system (CNS). This is reflected in tissue culture models of infection, where SINV infection of undifferentiated neurons resulted in high levels of viral replication and cell death, while infection of differentiated neurons resulted in low levels of viral replication and very little to no cell death [81].

The ability of mature neurons to resist SINV infection has been linked to the expression of a number of proteins, but as of yet, none of have been shown to confer complete protection from viral infection. Neuron differentiation has been shown to lead to changes in the expression of antiviral genes, and this is at least in part due to the increased expression of IRF3 and IRF7 [82]. Interferon response factors (IRFs) are transcription factors that activate interferon stimulated gene (ISG) expression in response to interferon (IFN) as well as induce IFN activation. While both IRF3 and IRF7 likely play important roles in enhancing antiviral signaling during infection, they are not solely responsible for restriction of viral replication in mature neurons, as silencing IRF7 expression in 
mature neurons did not restore viral replication to the same levels seen in immature neurons. In addition to expressing higher levels of antiviral genes, mature neurons also more readily respond to IFN- $\gamma$, which activates the JAK/STAT signaling pathway and induces the expression of multiple ISGs that reduce SINV replication $[83,84]$. Another important difference between mature and immature neurons is the expression of apoptosis-modulating proteins. In particular, Bcl-2 and Bax were found to inhibit SINV-induced apoptosis and their expression is correlated with survival [85]. Over expression of the apoptotic proteins $\mathrm{Bcl}-2$ and $\mathrm{Bax}$ in weanling mice resulted in lower mortality, suggesting that they protect neurons associated with survival. However, expression of these two proteins did not prevent the death of spinal cord motor neurons or hind limb paralysis, indicating that there are other factors at play.

Viral-induced apoptosis of neurons during infection is thought to be the main mechanism by which inflammation is induced in the brain, ultimately leading to encephalitis and the death of the host. SINV, as well as the encephalitic alphaviruses in general, are thought to induce apoptosis in a number of ways in the CNS. One is through the fusion of the viral envelope with the host endosomal membrane can trigger apoptosis by activating sphingomyelinase, leading to the release of ceramides that induce apoptosis [86]. A second way that SINV may induce apoptosis in neurons is by decreasing NA+K+-ATPase activity, resulting in the depolarization of the cell membrane and an influx of toxic levels of $\mathrm{Ca} 2+$, inducing cell lysis [87]. The death of neurons in the brain by viral infection leads to the release of pro-inflammatory pathogen- and damage-associated molecular 
patterns (PAMPs and DAMPs) as well as glutamate. The release of glutamate in particular can result in the death of neighboring infected and uninfected neurons. Excessive activation of the glutamate receptor leads to an influx of $\mathrm{Ca} 2+$, inducing excitotoxic cell death, which releases more PAMPs, DAMPs, and glutamate, and leads to further bystander death [86, 87].

The release of PAMPs and DAMPs from infected cells as well as the production of pro-inflammatory cytokines and chemokines results in the infiltration of immune cells into the brain, namely macrophages and T-cells [88]. In addition to the killing of neurons via direct viral infection, pathogenesis is also thought to be the result of excessive inflammation caused by the host antiviral immune response, as initiation of viral clearance and the inflammatory response correlates with the onset of neurological symptoms [89]. In particular, T-cells are thought to play a large role in exacerbating inflammation in the brain and their infiltration into the brain has been linked with fatal encephalomyelitis. This conclusion comes from studies which showed that mice deficient for $\alpha \beta$ T cells had increased survival compared to wild type mice when infected with neurovirulent SINV $[90,91]$. Therefore, controlling the inflammatory response in the brain so that viral clearance still occurs without damage to surrounding tissue is a critical aspect of determining lethality of encephalitic alphaviral disease.

Clearance of virus from the brain occurs in a primarily noncytolytic manner in order to prevent further damage to neural tissue. This process is largely mediated by IFNy produced from T cells as well as antibodies produced by Bcells against the viral glycoproteins [92, 93]. In particular, the B-cell production of 
antibodies which suppress viral infection is important for continued clearance of virus, as the viral RNA can persist in neurons for years following infection and has been shown to be capable of reactivating viral replication in mice deficient of B-cells $[84,94,95]$.

\section{Alphaviral Pathogenesis Traits}

Using both cellular and animal models of alphaviral disease, several key determinants of alphaviral pathogenesis have been identified. These virulence traits span both the viral proteins and the viral RNAs themselves and serve to both evade the induction of and blunt the efficacy of the innate immune response to infection. As the alphaviruses are highly sensitive to the effects of the Type-I IFNs, evading the host innate immune response is critical to alphaviral infection and pathogenesis. This section will detail the known alphavirus virulence traits.

\section{Virulence Traits of the Nonstructural Proteins}

In addition to its roles during alphaviral replication, the nsP1 protein is known to be important for alphaviral pathogenesis and multiple residues in nsP1 have also been identified as determinants for alphaviral virulence. In particular, palmitoylation of nsP1 is known to be important for viral pathogenesis as mutations in nsP1 that prevented palmitoylation were also found to impact neurovirulence in mice. Specifically, when the palmitoylated residues in nsP1 were mutated to alanine in Semliki Forest Virus (SFV), was found to replicate in mice and produce viremia but would no longer traffic to the brain and completely 
ablated mortality [96]. Apart from palmitoylation, a set of six mutations in nsP1 of RRV was found to attenuate viral infection in mice due to reduced viral replication in specific tissues. More specifically, RRV with these $6 \mathrm{nsP} 1$ mutations was found to have decreased replication in the skeletal muscle tissue and was more sensitive to type I IFN $[97,98]$. In addition to altered pathogenesis in vivo, the RRV nsP1 mutant was also shown to have decreased production of noncapped genomic RNA during viral replication; however, how this may have contributed to the virus's attenuation in vivo was unknown [99]. Finally, cleavage of nsP1 from nsP2 has also been identified to be a major determinant of virulence. The SINV T538I mutation in nsP1 is known to affect virulence by altering processing of the nonstructural polyprotein and the virus's sensitivity to interferon. The substitution of isoleucine for threonine at position 538 in nsP1 was found to decrease the speed of polyprotein processing and significantly affect neurovirulence [100, 101]. Mice infected with the SINV that had a threonine at position 538 developed severe neurological symptoms and high mortality rates. In contrast, those infected with SINV that had an isoleucine at position 538 only experienced mild disease and no mortality [102]. This has been attributed to the altered viral sensitivity to IFN due to the slowed processing of nsP2 preventing it from inhibiting the JAK-STAT signaling pathway.

nsP2 is known to play several roles apart from its involvement in viral replication which are critical for alphaviral pathogenesis. This includes inhibiting the JAK-STAT signaling pathway mentioned above, which is a major means by which production of antiviral effectors is induced. Binding of IFN to the IFN 
receptor results in the autophosphorylation of the associated kinases JAK1 and Tyk2 for the type I IFN receptors or JAK1 and JAK2 for the type II IFN receptors. The kinases in turn then phosphorylate the IFN receptor, which leads to the recruitment of the STAT proteins. The STAT proteins are then phosphorylated and released as dimers that then go to the nucleus to activate the transcription of interferon stimulated genes (ISGs). During alphaviral infection, nsP2 inhibits this pathway by disrupting the translocation of STAT1 to the nucleus, thus preventing STAT1 from binding to the DNA and inducing transcription of ISGs [103, 104]. As stated previously, the inhibition of the JAK-STAT pathway is known to be important for virulence, as the T538I mutation in nsP1 which alters this activity of nsP2 results in decreased pathogenesis. In addition to inhibiting the JAK-STAT pathway, nsP2 is also known to be responsible for host shutoff. In the arthritogenic alphaviruses, shutoff of host transcription occurs through translocation of nsP2 to the nucleus, where it induces degradation of RNA polymerase II subunit RPB1 [45]. nsP2 is also thought to play an important role in the shutoff of host translation as well, although the exact mechanism is not yet understood [105]. Host shutoff has been linked to viral cytopathogenicity, as a P726G mutation in nsP2 which prevents shutoff of host transcription results in decreased cell death and viral persistence in mammalian cells in vitro [106]. In addition to inhibiting host immunity, transcription, and translation, modulating the host stress response is also an important aspect of alphaviral infection. Viral infection can trigger the formation of stress granules within the host, resulting the in the sequestration of host translation machinery and an 
arrest in both host and viral protein synthesis. Alphaviruses are known to suppress stress granule formation through the ADP-ribosylhydrolase activity of nsP3 [47]. By removing ADP-ribose from ADP-ribosylated proteins present in stress granules, nsP3 is able to disassemble the stress granules that form in response to infection. This activity of nsP3 is known to be important for efficient viral replication and thus for pathogenesis as well. Mutations in nsP3 of CHIKV which abolish hydrolase activity results in virus that is unable to replicate in either mammalian or mosquito cells. Similarly, reducing the nsP3's ability to bind ADPribose results in slower viral replication in tissue culture as well as significantly reduced mortality in 2-day old mice, illustrating the importance of nsP3 ADPribosylhydrolase activity to alphaviral virulence [107].

\section{Virulence Traits of the Structural Proteins}

There have also been several virulence determinants identified in the structural proteins in addition to the nonstructural proteins. In the encephalitic alphaviruses, capsid is responsible for shutoff of host transcription rather than nsP2. In contrast to the arthritogenic viruses, shutoff of host transcription by the encephalitic alphaviruses utilizes capsid, which blocks nucleocytoplasmic trafficking in the host cell [108]. Similar to what occurs in the arthritogenic alphaviruses, host shutoff of transcription by the encephalitic alphaviruses is also associated with cytopathogenicity, as mutations in capsid which prevent host shutoff also results in decreased cell death [109]. In addition to the capsid protein, the viral glycoproteins have also been shown to be important for 
virulence, particularly in regard to viral entry. There are multiple residues in the viral glycoproteins that are known to affect disease severity. In SINV, development of neurovirulence has been specifically association with mutations in E1 and E2, which are responsible for membrane fusion and receptor binding respectively [110]. Mutations in the glycoproteins have also been shown to affect host range, tissue/cell tropism, cell attachment, and immunogenicity [111-114].

\section{Virulence Traits of the Alphavirus RNAs}

In addition to the above protein-based virulence traits, there are multiple features of the viral RNA that have been characterized as key determinants of alphaviral virulence. Several contribute to viral infection by preventing detection of the viral RNA by innate immune sensors. A major way that the host cell differentiates self from non-self RNA is through the addition of a modified cap structure to the 5' end of mRNA. In particular, RIG-I detects RNA in the cytoplasm which have a 5' tri- or diphosphate instead of the proper cap structure. Once RIG-I binds to a noncapped RNA, it interacts with MAVS and starts a signaling cascade which leads to the induction of type I IFN. In order to avoid detection by RIG-I, alphaviruses add a type 0 cap to the 5' ends of the VRNA. However, this cap differs from the host type 1 cap in that it is not 2'O methylated. RNA that lack the 2'O methylation of the 5' cap can be sensed by IFIT1, which can bind to the RNA and block translation. To avoid detection by IFIT1, alphaviruses have a stable stem-loop in the 5'UTR which prevents IFIT1 from binding the vRNA [115]. This structure has been shown to be important for 
virulence in multiple alphaviruses as disruption of the stem-loop with point mutations in the 5 'UTR, especially at nucleotides 3,5 , and 8 , results in attenuated neurovirulence in mice [115-118].

In addition to the viral cap structure and secondary structure in the 5'UTR, the Opal stop codon between nsP3 and nsP4 is also a determinant for alphaviral virulence. However, how the Opal stop codon impacts viral pathogenesis changes depending on the alphavirus. Mutation of the opal stop codon in CHIKV results in reduced pathogenicity as mice infected with virus where the Opal codon has been replaced with arginine experience decreased footpad swelling and reduced inflammation in the joint [119]. In contrast, introduction of the opal stop codon in SFV lead to significant attenuation of disease in adult mice [120]. There is also some evidence that the importance of the Opal stop codon to virulence may be dependent on the host. In O'nyong'nyong virus (ONNV), the presence of the Opal stop codon results in increased viral replication in BHK cells, but drastically decreased replication in C6/36 mosquito cells [121]. While the mechanism by which the Opal stop codon impacts virulence has not been characterized, it is thought to play a role in controlling the amount of nsP4 which is produced [122].

\section{Noncapped Genomic Alphaviral RNA}

While capping of the genome is crucial for viral replication, alphaviral RNA are not universally capped and the rate at which the VRNA is capped changes depending on the host. Furthermore, both capped and noncapped vRNA are 
packaged into viral infection, despite the fact that the noncapped genomes cannot be translated and are essentially noninfectious [99]. During vertebrate infection, SINV produces and packages approximately four-fold more noncapped genomic RNA (ncgRNA) than capped genomic RNA (Fig. 1.4A). In contrast, SINV infection of mosquito produces capped and ncgRNA in approximately equal parts. Additionally, it is known that different amounts of ncgRNA are produced at different points during infection. More specifically, in mammalian tissue culture models of infection, capping efficiency improves and ncgRNA production decreases as infection progresses (Fig. 1.4B). Regardless, this overwhelming presence of ncgRNA during viral infection suggests that they play an important role in alphaviral infection. While the specifics of what the ncgRNA do during infection are poorly understood, they are known to be important for proper viral infection. As mentioned in the previous section, a RRV mutant that was attenuated in mice was found to produce less ncgRNA compared to the WT RRV $[97,99]$. Additionally, the presence of the ncgRNA during infection of mammalian cells is known to be correlated with the activation of type I interferon [99]. Noncapped genomic RNA are also known to be important for alphaviral infection of mosquitos, as particles that contained primarily ncgRNA where able to more efficiently initiate infection in the midgut than those containing capped genomic RNA [123].

Altogether, these findings suggest that the production of ncgRNA is linked to alphaviral infection and pathogenesis. However, there has yet to be a study which directly links ncgRNA production to the virus's ability to replicate and 
produce disease. This is partially due to the lack of a good system by which ncgRNA production can be reliably altered for the entire course of infection. Mackenzie-Liu et al where able to separate particles containing capped RNA from those containing ncgRNA by density, as the capped RNA correlated with heavier particles. However, this method only changes the proportion of capped and ncgRNA present during the initial round of viral infection, as the ratios of capped to ncgRNA will return to wild type after one round of replication. Thus, one of the primary goals of this project was to establish a means by which ncgRNA production could be altered that would last for the entirety of infection.

Characterization of residues in nsP1 that impact vRNA capping have largely focused on residues that abolish either the methylatransferase or guanylyltransferase activities of nsP1. Mutation of these residues is lethal to the virus, making them unsuitable for studying the functions of ncgRNA during viral infection. However, in 2015 a study by Li et al reported a number of resides in the nsP1 of VEEV that, when mutated, where capable of decreasing or increasing cap formation to various degrees. Among them, alanine substitutions at positions 285 and 375 decreased capping efficiency by $\sim 10$ and 2 fold respectively compared to wild type nsP1. Additionally, an alanine substitution at the 354 position increased capping efficiency by $\sim 3.5$ fold [124]. This was the first time that it was shown that it was possible to increase the capping activity of nsP1. The Y285, N375, and D354 residues in VEEV are very highly conserved among both the arthritic and encephalitic alphaviruses, making it likely that mutation of these residues in other alphaviruses will result in a similar effect on capping 
efficiency. Additionally, all three of these residues occur at interesting locations in the recently described structure of nsP1 (Fig 1.5). The Y285 residue falls within the capping domain of nsP1 and is known to be involved in binding GTP for methylation. While the specific location of the N375 residue has not been resolved, both it and the D354 residue occur in structures that extend out from the waist of the enzyme and into the pore formed by the nsP1 ring [33]. It is worth noting that it is currently unknown whether nsP1 is enzymatically active when forming the ring complex, therefore the location of the N375 and D354 residues within the structure of nsP1 may differ when nsP1 is active and part of the replication complex. Overall, the location of these residues in nsP1, their highly conserved nature, and their ability to affect nsP1 capping efficiency, and therefore ncgRNA production, made the Y285A, N375A, and D354A mutations in particular good candidates for determining the importance of ncgRNA to biological infection.

\section{$\underline{\text { Rationale }}$}

Alphaviruses are known to produce significant quantities of viral genomic RNAs that lack the canonical viral 5' cap structure. These noncapped genomic RNA are known to be packaged into viral particles, despite the fact that the noncapped genomes cannot be translated and are essentially noninfectious [99]. Furthermore, previous studies suggest that ncgRNA production may be a determinant of alphaviral pathogenesis [97, 99]. While these findings suggest that the ncgRNA are important for alphaviral infection, the role that the ncgRNA 
play during viral infection and the development of viral pathogenesis had yet to be characterized. This led to the hypothesis that the ncgRNAs were critical to efficient alphaviral infection and the development of pathogenesis. To determine the role of the ncgRNA during alphaviral infection, we developed a set of nsP1 mutants in SINV that had either increased or decreased RNA capping efficiency, which would lead to consistently altered ncgRNA production during infection. We then used these nsP1 capping mutants to characterize the impact of altered ncgRNA production on viral infection at the molecular level in cellular models of infection (Chapter 2) as well as on viral pathogenesis using animal models of infection (Chapter 3). Defining the role of the ncgRNA during alphaviral infection will not only expand our knowledge by characterizing a novel alphaviral RNA species, but will also give insight to the development of novel antiviral strategies with which alphaviral disease may be treated or prevented. 


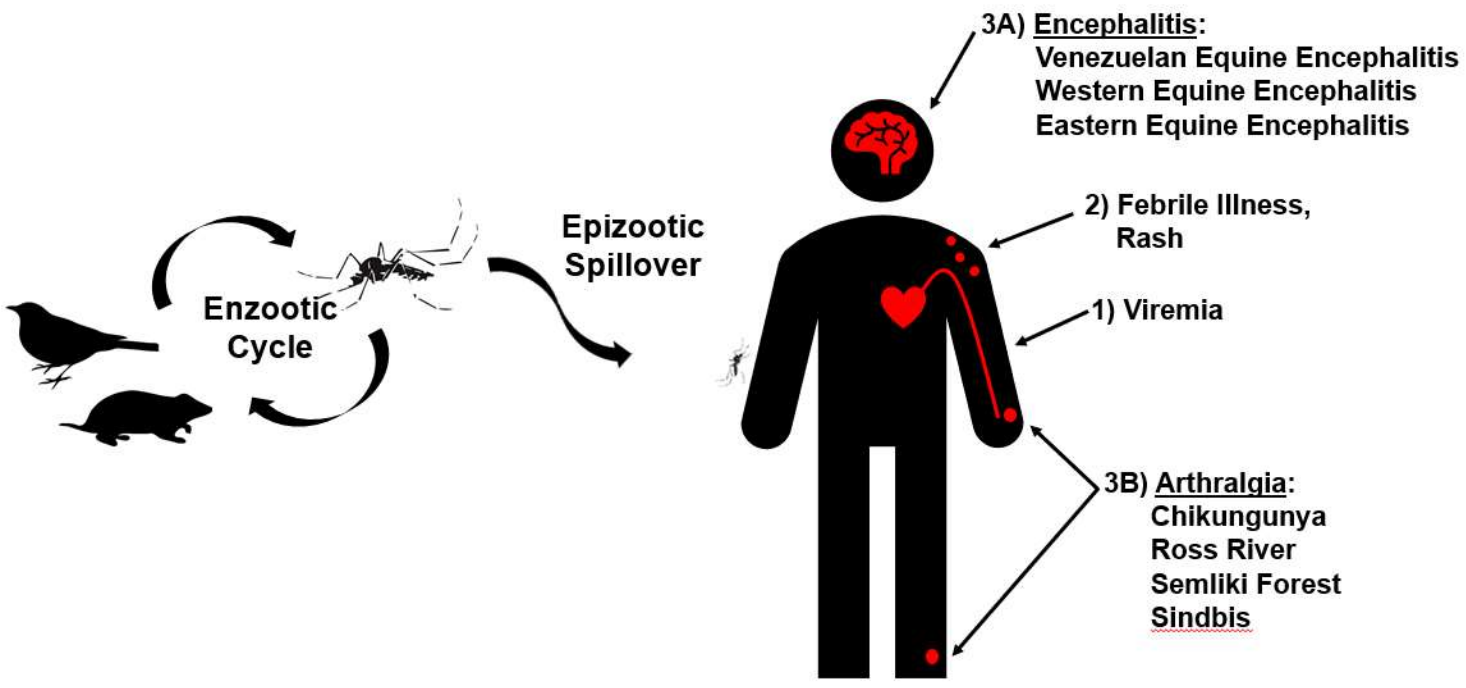

Figure 1.1. Alphaviral transmission and disease. The natural transmission cycle of alphaviruses is between an invertebrate vector and a vertebrate host. Epizootic spillover events can occur that results in the infection of humans. Once the virus is transmitted from the mosquito vector to a human host, the virus begins to replicate resulting in high titer viremia as well as febrile illness and rash. Depending on the alphavirus, the disease then has the potential to develop into either potentially lethal encephalitis or debilitating arthritis. Examples of encephalitic alphaviruses include VEEV, WEEV, and EEV, while the arthritogenic alphaviruses include CHIKV, RRV, SFV, and SINV. 


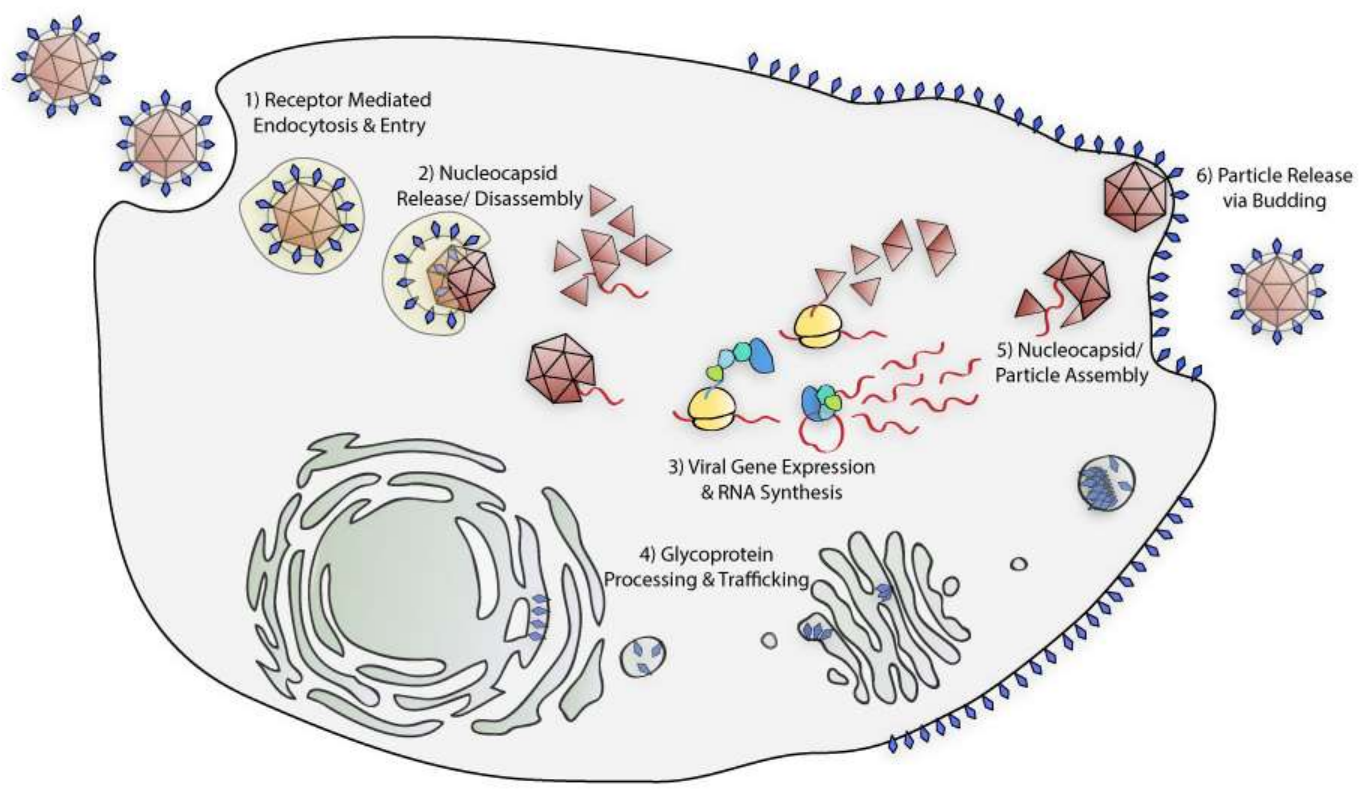

Figure 1.2. The alphaviral life cycle. 1) Alphaviral infection begins with the viral particle binding to receptors on the surface of the host cell and entering via receptor mediated endocytosis. 2) After entry, the viral envelope fuses with the endosomal membrane leading to release of the nucleocapsid core, which is then disassembled to release the viral genome. 3) The viral genome is then translated to produce the nonstructural proteins, which are processed to form the viral replicase. The viral replicase then synthesizes the negative strand RNA template as well as the subgenomic RNA. The subgenomic RNA is then translated to produce the structural proteins, which include the glycoproteins and capsid. 4) The glycoproteins are processed in the endoplasmic reticulum and then trafficked to the cell surface. 5) Capsid associates with the newly synthesized genomic RNA to form the nucleocapsid core. 6) Newly formed viral particles are released from the cell via budding. 


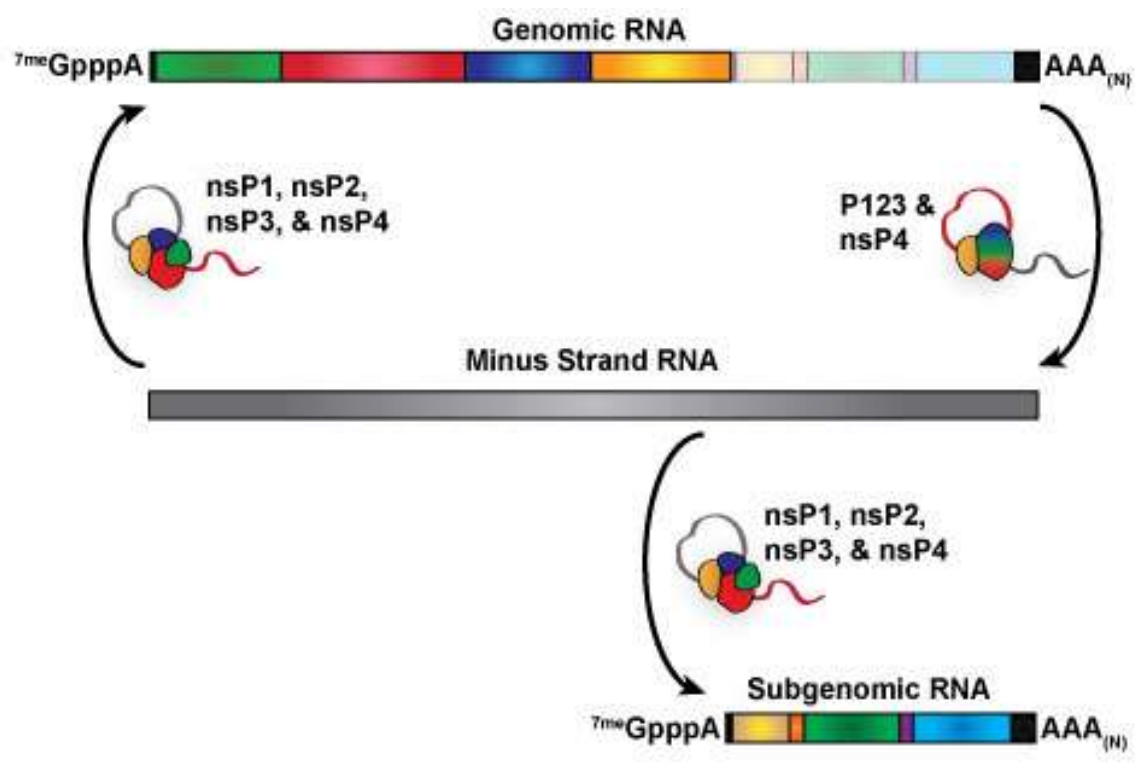

Figure 1.3. Alphaviral RNA replication and genetic organization. Translation of the viral genome produces the nonstructural polyprotein P1234. nsP4 is first cleaved off and associated with P123 in order to form the complex that is responsible for synthesizing the minus strand RNA template. P123 is then further processed into the individual nonstructural proteins nsP1, 2, and 3 . The four fully processed nonstructural proteins then form the replication complex that is responsible for synthesizing the genomic and subgenomic RNA. 


\section{Encapsidated SINV Genomic RNA}

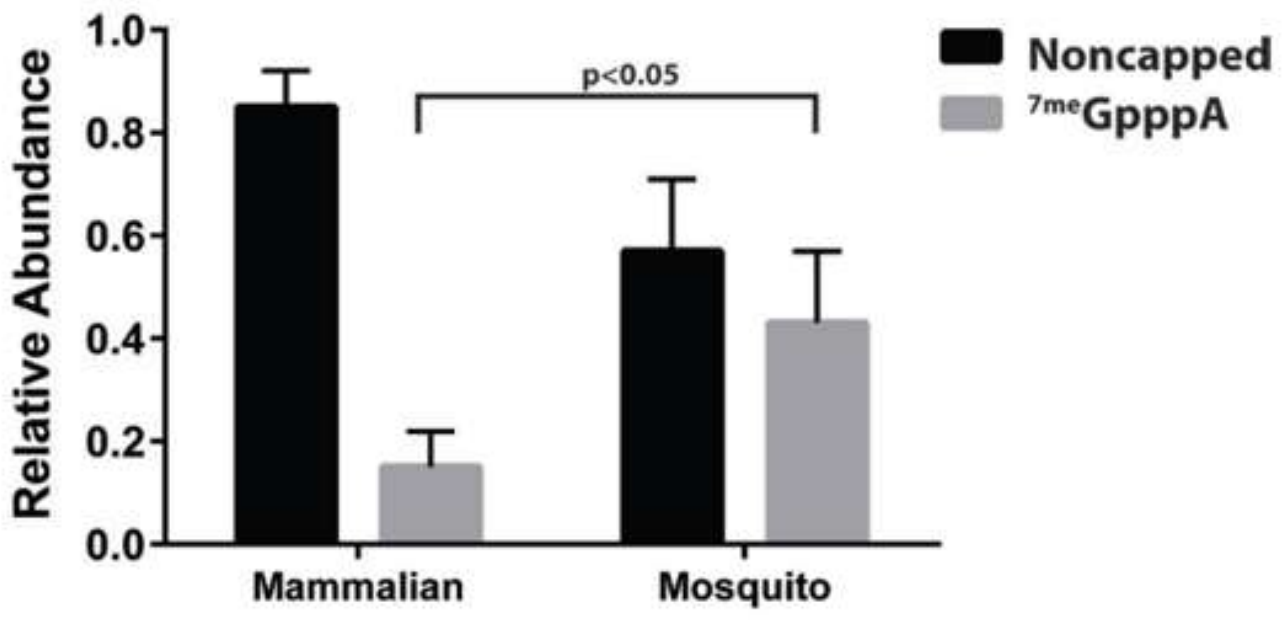

B

\section{Encapsidated SINV Genomic RNA}

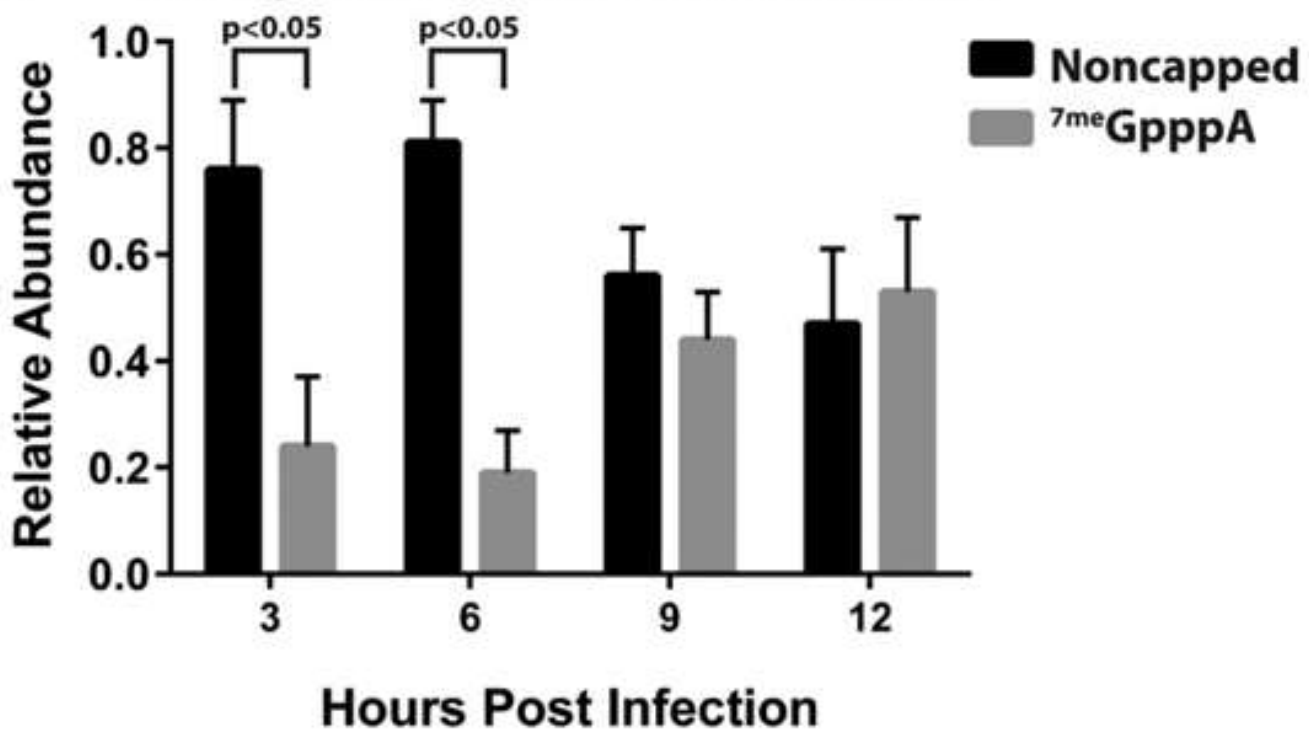

Figure 1.4. Capped and noncapped genomic RNA are produced during alphaviral infection. (A) Quantitative assessment of the 5' terminus of encapsidated SINV genomic RNAs from particles produced during infection of mammalian (BHK-21) and mosquito (C6/36) tissue culture cells. (B) Quantitative assessment of the encapsidated SINV genomic RNAs from particles produced in 
the mammalian 293HEK tissue culture cell line over time. Data shown are the means of at least 3 independent replicates, with error bars representing the standard deviation of the mean. Adapted from Sokoloski et al, 2015 [89]. 


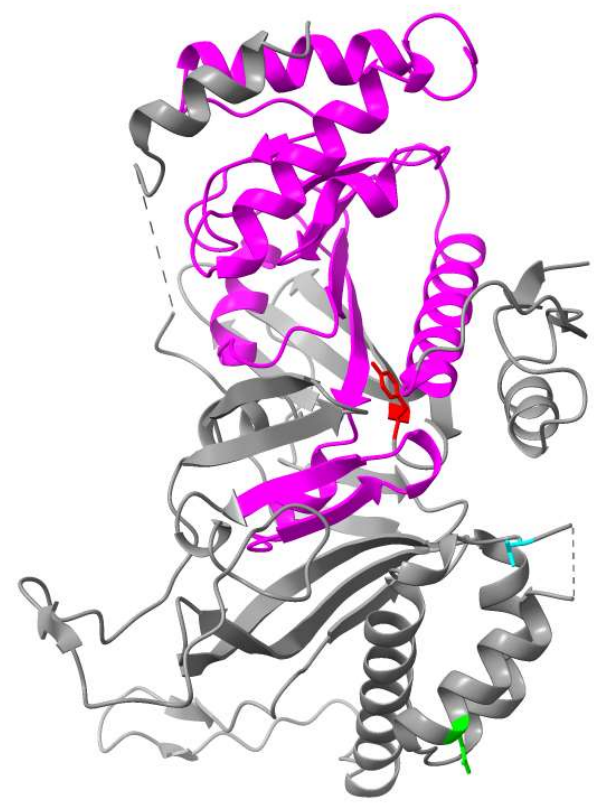

Figure 1.5. Structure of nsP1. Amino acid residues of importance are highlighted as follows: red, Y286; green, D355; blue, N376; pink, capping domain. PDB-6Z0V. 


\section{CHAPTER 2}

\section{INCREASING THE CAPPING EFFICIENCY OF THE SINDBIS VIRUS NSP1 PROTEIN NEGATIVELY AFFECTS VIRAL INFECTION ${ }^{1}$}

\section{Summary}

Alphaviruses are arthropod-borne RNA viruses that are capable of causing severe disease and are a significant burden to public health. Alphaviral replication results in the production of both capped and noncapped viral genomic RNAs, which are packaged into virions during the infections of vertebrate and invertebrate cells. However, the roles that the noncapped genomic RNAs (ncgRNAs) play during alphaviral infection have yet to be exhaustively characterized. Here, the importance of the ncgRNAs to alphaviral infection was assessed by using mutants of the nsP1 protein of Sindbis virus (SINV), which altered the synthesis of the ncgRNAs during infection by modulating the protein's capping efficiency. Specifically, point mutants at residues Y286A and N376A decreased capping efficiency, while a point mutant at D355A increased the capping efficiency of the SINV genomic RNA during genuine viral infection. Viral growth kinetics were significantly reduced for the D355A mutant relative to wild

\footnotetext{
1 This chapter is adapted from work published in mBio, 2018, Volume 9, Issue 6 with the same title under a Creative Commons Attribution (CC BY) license 125. LaPointe, A.T., et al., The Identification and Characterization of Sindbis Virus RNA:Host Protein Interactions. J Virol, 2018.
} 
type infection, whereas the Y286A and N376A mutants showed modest decreases in growth kinetics. Overall genomic translation and nonstructural protein accumulation was found to correlate with increases and decreases in capping efficiency. However, genomic, minus strand, and subgenomic viral RNA synthesis was largely unaffected by the modulation of alphaviral capping activity. In addition, translation of the subgenomic vRNA was found to be unimpacted by changes in capping efficiency. The mechanism by which decreased presence of ncgRNAs reduced viral growth kinetics was through the impaired production of viral particles. Collectively, these data illustrate the importance of ncgRNAs to viral infection and suggests that they play in integral role in the production of viral progeny.

\section{Introduction}

Alphaviruses are positive sense, single-stranded RNA viruses which are capable of being transmitted between sylvatic vertebrate reservoir hosts and competent mosquito vectors in an enzootic cycle. During epizootic events, these viruses are capable of causing severe disease and are broadly categorized into two groups based on symptomology. Disease caused by the encephalitic alphaviruses, such as Venezuelan Equine Encephalitis virus, are characterized by neurological symptoms and are capable of causing severe encephalitis, which may result in the death of the vertebrate host $[11,12]$. In contrast, the arthritogenic alphaviruses, such as Sindbis virus (SINV), Ross River virus (RRV), and Chikungunya (CHIKV), cause disease ranging in severity from mild febrile 
illness to debilitating polyarthritis, which may persist for several months to years past the acute phase of infection $[3,5,126-128]$. Despite the burden to public health posed by the alphaviruses, there are currently no effective and safe antiviral treatments for alphaviral disease.

During alphaviral infection, three viral RNA species are produced: the genomic strand, which encodes the nonstructural polyprotein, which is processed proteolytically to form the replication machinery; a minus strand RNA template; and a subgenomic RNA, which encodes the structural proteins. The positive sense alphaviral RNAs (vRNAs), namely the genomic and subgenomic vRNAs, have a type-0 cap structure co-transcriptionally added to their 5 ' ends during RNA synthesis in order to protect the 5 ' end of the transcript and to facilitate translation after entry to the host cell [129-131]. The addition of the 5' cap structure to the positive sense vRNAs is a multistep process involving at least two alphaviral nonstructural proteins. Briefly, the methyltransferase domain of the alphaviral nsP1 protein catalyzes the addition of a methyl group from Sandenosylmethionine to a GTP molecule, forming a covalent $\mathrm{m}^{7} \mathrm{GMP}-\mathrm{nsP} 1$ intermediate [132]. Following the removal of the 5' y-phosphate on the nascent vRNA molecule by nsP2, the $\mathrm{m}^{7} \mathrm{GMP}$ moiety is then transferred to the vRNA molecule by the guanylyltransferase activities of nsP1, resulting in the formation of the type-0 cap structure, ${ }^{7 m e}$ GpppA $[40,133]$. Importantly, biochemical studies of alphaviral nsP1 proteins have found that both the methyltransferase and guanylyltransferase activity of nsP1 can be altered either negatively or positively via point mutations in vitro $[35,124,134]$. 
The presence of the type-0 cap structure on the alphaviral genomic and subgenomic vRNAs were first observed by paper electrophoresis, or thin-layer chromatography, of metabolically labeled vRNAs that were chemically and enzymatically degraded $[129,130,135]$. These studies further identified the sequences of the 5 ' termini of the alphaviral genomic and subgenomic vRNAs, which provided the first evidence for independent promoter initiation for the two positive-sense vRNA species [130]. While these studies were able to identify the presence of the 5' type-0 cap structure, they were, by the nature of their design and technological limitations, unable to determine the relative frequency with which the positive sense vRNAs were capped.

Recently, we reported findings that indicated that the alphaviral genomic vRNAs are not ubiquitously capped, and that a significant proportion of the genomic vRNAs produced during SINV and RRV infection lack the 5' type-0 cap structure [99]. Furthermore, analyses of infectious and noninfectious viral particles demonstrated that both the capped and noncapped genomic VRNAs are packaged into viral particles throughout the course of infection. Through the use of tissue culture models of alphaviral infection, the presence of the noncapped vRNAs were found to correlate with the activation of a type-I IFN response. Moreover, an attenuated RRV mutant was found to produce fewer noncapped genomic RNAs relative to wild type virulent RRV [97, 99]. Collectively, these data were highly suggestive of an important biological role for the noncapped genomic vRNAs during infection; however, the precise functions of the noncapped genomic vRNAs during infection remained unknown. 
The goal of this study was to determine the importance of noncapped vRNAs to SINV infection of tissue culture cells. Here, we show that the capping activity of SINV nsP1 is capable of being modulated, both positively and negatively, via the mutation of specific residues within the Iceberg Domain, a structurally organized domain of cryptic function that is conserved amongst many viral capping enzymes (12). In addition, SINV infection was determined to be more detrimentally impacted by increasing the capping efficiency of SINV nsP1, rather than by decreasing efficiency. Specifically, increasing the rate of alphaviral capping negatively impacts viral growth kinetics by negatively affecting viral particle production. Collectively, our findings indicate that the noncapped vRNAs do play an important role during viral infection, and that decreasing the presence of noncapped vRNA impacts the viral life cycle at a fundamental level.

\section{Materials and Methods}

\section{Tissue Culture Cells}

Baby hamster kidney 21 (BHK-21) fibroblast cells (a gift from R.W. Hardy, Indiana University- Bloomington) were maintained in Minimal Essential Media (MEM, Cellgro) containing 10\% fetal bovine serum (FBS, Atlanta Biologicals), $1 \%$ antibiotic-antimycotic solution (Cellgro), 1\% nonessential amino acids (Cellgro), and $1 \%$ L-glutamine (Cellgro). Cells were cultured at $37^{\circ} \mathrm{C}$ and at $5 \% \mathrm{CO}_{2}$ in a humidified incubator. Low passage stocks were maintained via regular passaging using standard subculturing techniques. 


\section{Generation of SINV Capping Mutants}

The SINV nsP1 mutants used in this study were generated either by site directed mutagenesis, or Gibson assembly reactions. Particularly, the SINV p389 Y286A and D355A mutants were generated via site-directed mutagenesis according to the instructions for Q5 site-directed mutagenesis kit (NEB). To this end, the parental wild type strain consisting of the p389 SINV nsP3-GFP reporter strain was PCR amplified with high fidelity Q5 DNA polymerase using primer sets that incorporated the indicated alanine substitutions, individually. Due to technical limitations that prevented the successful use of site-directed mutagenesis, the N376A mutant was generated by Gibson assembly via the Gibson Ultra kit (SGI) through the use of restriction digested p389 cDNA plasmid and a synthetic DNA fragment, according to the manufacturers' instructions. SINV nanoluciferase reporters mutants, similar to those previously described, were generated by either site-directed mutagenesis, or by conventional restriction enzyme cloning schemes which swapped the GFP coding region of the existing p389 site mutants with a Nanoluciferase coding region in a modular fashion [99].

In all cases, individual mutants were verified by whole genome sequencing before proceeding to subsequent analyses. Full genome sequences are available upon request. Highly similar phenotypes were observed for any given mutant in all virus backgrounds evaluated.

\section{Production of SINV Wild Type and Mutant Virus Stocks}


Wild type, Y286A, D355A, and N376A SINV p389 (a derivative of the Toto1101 strain containing GFP in frame with nsP3 [136]), as well as SINV pToto1101-nanoluc (a derivative of the Toto1101 strain containing nanoluciferase in frame with nsP3 [99]), were prepared by electroporation as previously described $[125,137]$. Briefly, $2.8 \times 10^{6}$ BHK-21 cells were electroporated with $10 \mu \mathrm{g}$ of in vitro transcribed RNA using a single pulse at $1.5 \mathrm{kV}, 25 \mathrm{~mA}$, and 200Ohms from a Gene Pulser Xcell system (BioRad) as previously described [125]. Cells were then incubated at normal incubation conditions until cytopathic effect became apparent, at which point the supernatant was collected and clarified by centrifugation at $8,000 \times \mathrm{xg}$ for $10 \mathrm{~min}$ at $4^{\circ} \mathrm{C}$. The clarified supernatant was then aliquoted into small-volume stocks, which were then stored at $-80^{\circ} \mathrm{C}$ for later use.

\section{Analysis of Viral Growth Kinetics}

To determine if mutation of the SINV nsp1 protein negatively affected infection, the viral growth kinetics of each of the individual strains described above were assayed in tissue culture models of infection. Essentially, BHK-21 cells were seeded in a 24-well plate and incubated under normal conditions. Once cell monolayers were $80-90 \%$ confluent, they were infected with either the wild-type parental virus, or the individual capping mutant viruses, at an $\mathrm{MOI}$ of 5 infectious units (IU) per cell. After a $1 \mathrm{hr}$ adsorption period, the inoculum was removed and the cells were washed twice with $1 \times$ PBS to remove unbound viral particles. Whole media was added, and the cells were incubated at $37^{\circ} \mathrm{C}$ in a 
humidified incubator in the presence of $5 \% \mathrm{CO}_{2}$. At the indicated times post infection, tissue culture supernatants were harvested (and the media replaced), and viral titer was determined via plaque assay.

\section{Quantification of Infectious Virus by Plaque Assay}

Standard virological plaque assays were used to determine the infectious titer of all viral samples produced during these studies. Briefly, BHK-21 cells were seeded in a 24-well plate and incubated under normal conditions. Once the cell monolayers were $80-90 \%$ confluent, they were inoculated with 10 -fold serial dilutions of virus-containing samples. After a $1 \mathrm{hr}$ adsorption period, cells were overlaid with a solution of $0.5 \%$ Avicel (FMC Corporation) in $1 \times$ media for 28 30hrs [138]. The monolayers were fixed with formaldehyde solution $(3.8 \%$ formaldehyde in 1xPBS) for a period of no less than one hour. Plaques were visualized via staining with crystal violet after removal of the overlay and quantified by manual counting.

\section{XRN1 Protection Assay/ RppH Assay}

Viral genomic RNAs were extracted from purified viral particles harvested at $24 \mathrm{hpi}$. After extraction, the RNA samples were subjected to enzymatic degradation via the $5^{\prime} \rightarrow 3^{\prime}$ exonuclease $X R N-1$, which is capable of degrading 5 ' monophosphate and RNAs in vitro, but is unable to effectively degrade RNA substrates that are 5' capped $[139,140]$. Briefly, equivalent amounts of viral genomic RNAs were incubated in the presence of 0.25 units of XRN-1 in a final 
volume of $20 \mu \mathrm{l}$ for a period of 5 minutes at $37^{\circ}$ prior to being quenched via the addition of High Salt Column Buffer (25mM Tris-HCl, pH 7.6 / 400mM NaCl / $0.1 \% \mathrm{w} / \mathrm{v}$ Sodium Dodecyl Sulfate (SDS). After the termination of the reaction, the RNAs were purified via phenol:chloroform extraction and ethanol precipitation with linear acrylamide carrier. The resulting precipitate was resuspended and utilized as the substrate for the synthesis of cDNA via ProtoScript II reverse transcriptase (NEB). The resulting cDNAs were quantified via qPCR of an amplicon located in the nsP1 region, as described below, to determine the sensitivity of a sample relative to wild type viral genomic vRNAs [125, 141].

To confirm that the nature of the $5^{\prime}$ end, in particular the presence of a $5^{\prime}$ cap structure, was responsible for the differences in XRN-1 sensitivity the extracted RNAs were co-incubated with XRN-1 in the presence of the decapping enzyme RppH [99, 142, 143]. To this end, the above reactions were supplemented with 1.25 units of $\mathrm{RppH}$ and processed as described above.

\section{Metabolic Labeling of Protein Synthesis}

To determine the rates of host and viral during infection, BHK-21 cells were seeded in a 12-well tissue culture dish and grown to $80-90 \%$ confluence prior to being infected with either wild type parental virus, or one of the individual capping mutant viruses at an $\mathrm{MOI}$ of $10 \mathrm{IU} /$ cell. After a $1 \mathrm{hr}$ adsorption period, fresh media was added to each well, and the cells were incubated under their normal incubation conditions. Thirty minutes before the indicated times post infection, the media was removed and replaced with methionine- and cysteine- 
free DMEM (Cellgro) to starve the cells of methionine. After a 30 minute incubation period, the starvation media was removed and replaced with methionine- and cysteine-free DMEM supplemented with $50 \mu \mathrm{M} \mathrm{L-}$ azidohomoalanine (L-AHA), a methionine analogue [144, 145]. After 2hrs, the labeling media was removed, the cells were washed with 1x PBS, and then harvested with RIPA buffer (50mM Tris-HCl, $\mathrm{pH} 7.5$ / 150mM NaCl / 1\% v/v Nonidet P-40 (NP-40) / 0.5\% w/v SDS / 0.05\% w/v Sodium Deoxycolate / 1mM EDTA). Cell lysates were labelled with DIBO-Alexa 648 (Invitrogen) at a final concentration of $5 \mu \mathrm{M}$ and incubated at room temperature in the dark for at least 1 hour. The labeled lysates were then loaded onto a $12 \%$ SDS-PAGE gel, and the proteins were separated by electrophoresis. Fluorescence was then imaged using a Pharos FX Plus Molecular Imager (BioRad), and densitometry of individual protein species was used to quantify viral and host protein expression.

\section{Western Blot Detection of SINV nsP2 Protein Expression}

Whole cell lysates were generated from BHK-21 cells infected with either wild type, or individual SINV nsP1 capping mutant viruses by resuspension in RIPA buffer at 12 and $16 \mathrm{hpi}$. Equivalent amounts of protein were loaded onto $10 \%$ acrylamide gels, and the individual protein species were resolved using standard SDS-PAGE practices. After sufficient resolution of the protein species, the proteins were transferred to PVDF membranes, which were rinsed in methanol and thoroughly dried after transfer. After drying the blots were probed with anti-SINV nsP2 polyclonal sera (a gift from R.W. Hardy at Indiana 
University- Bloomington), or anti-Actin (clone mAbGEa, ThermoFisher Scientific) antibodies diluted in 1xPBS supplemented with 1.0\% v/v Tween (PBST) for a period of at least one hour at $25^{\circ} \mathrm{C}$ under gentle rocking. The blots were washed three times with 1xPBST, and probed with fluorescent anti-rabbit (A32732, ThermoFisher Scientific) and anti-mouse secondary (A32723, ThermoFisher Scientific) antibodies. Protein detection was achieved using a Pharos FX Plus Molecular Imager (BioRad), and densitometry of individual protein species was used to quantify viral and protein expression.

\section{qRT-PCR Detection of SINV vRNAs}

The quantitative detection of the SINV vRNAs was accomplished as previously described, with minor modifications [137]. Briefly, to quantify the SINV genomic, subgenomic, and negative sense vRNAs, BHK-21 cells were infected at an $\mathrm{MOI}$ of $5 \mathrm{IU} /$ cell and cells were harvested at 2, 4, and 8hpi via the addition of Ribozol (VWR). Total RNA was then isolated via extraction, and $1 \mu \mathrm{g}$ was reverse transcribed using Protoscript II Reverse Transcriptase (NEB) and a cocktail of specific RT primers based on the intended amplification targets. To detect the positive-sense RNA species the RT primer cocktail consisted of nsP1, $\mathrm{E} 1$, and $18 \mathrm{~S}$ reverse primers; and to detect the minus strand the RT cocktail consisted of nsP1 forward and $18 \mathrm{~S}$ reverse primers. The individual VRNA species were detected via TaqMan probes using the following primer sets: SINV nsP1 F 5'-AAGGATCTCCGGACCGTA-3', SINV nsP1 R 5'AACATGAACTGGGTGGTGTCGAAG-3'; SINV E1 F 5'- 
TCAGATGCACCACTGGTCTCAACA-3', SINV E1 R 5'-

ATTGACCTTCGCGGTCGGATACAT-3'; Mammalian 18S F 5'-

CGCGGTTCTATTTTGTTGGT-3', Mammalian 18S R 5'-

AGTCGGCATCGTTTATGGTC-3'. The sequences of the TaqMan detection

probes are as follows: SINV nsP1 Probe 5'-

ACCATCGCTCTGCTTTCACAACGA-3', SINV E1 Probe 5'-

ACTTATTCAGCAGACTTCGGCGGG-3', and Mammalian 18S Probe 5'-

AAGACGGACCAGAGCGAAAGCAT-3'.

To quantify the total number of viral particles present in a sample, BHK-

21 cells were infected at an $\mathrm{MOI}$ of $5 \mathrm{IU} /$ cell and supernatant was collected at 24hpi. As previously described, $5 \mathrm{uL}$ of supernatant was reverse transcribed [137]. Reverse transcription and qPCR reactions were performed identical to those above with the exception of the mammalian 18S primer and probes not being used. Absolute quantities of viral genomic RNAs were determined via the use of a standard curve of known concentration. To determine the amount of subgenomic vRNA present, the absolute quantities of RNA detected with the nsP1 primer set were subtracted from that detected with the E1 primer set, as the E1 primer set will detect both the genomic and subgenomic RNAs, but the nsP1 primer set will only detect the genomic RNA.

\section{Cell Viability Assay}

To determine the effect of SINV infection on cell viability, BHK-21 cells were seeded in a 96-well plate and were infected with the wild type parental virus 
or the individual capping mutant viruses at an $\mathrm{MOI}$ of $10 \mathrm{IU} /$ cell. After a $1 \mathrm{hr}$ adsorption period, whole medium was added and the cells were incubated under normal incubation conditions for the described times. Afterwards, the cells washed with 1x PBS and a solution of $1 / 6^{\text {th }}$ CellTiter 96 AQueous One Solution Reagent (Promega) in 1x PBS was added to each well. The cells were then allowed to incubate at $37^{\circ} \mathrm{C}$ and $5 \% \mathrm{CO}_{2}$ for $2 \mathrm{hrs}$. Absorbance at $490 \mathrm{~nm}$ was then recorded using a Synergy H1 microplate reader (BioTek).

\section{Quantification of Viral Gene Expression via Nanoluciferase Assays}

To quantify genomic translation during infection, BHK-21 cells were infected at an $\mathrm{MOI}$ of $5 \mathrm{IU} / \mathrm{cell}$ with the wild type parental virus or the individual capping mutants containing the nanoluciferase gene within the nsP3 protein [99]. After a $1 \mathrm{hr}$ adsorption period on ice, the inoculum was removed and the medium was replaced with pre-warmed whole medium. The infected cells were incubated under normal conditions, and at the indicated times post-infection, the medium was removed and the tissue culture cells were washed with 1x PBS. The cells were then harvested into a crude lysate by the addition of 1x PBS supplemented with $0.5 \%(\mathrm{v} / \mathrm{v})$ Triton $\mathrm{X}-100$. The lysate was transferred to a microfuge tube and frozen until the completion of the experimental time course. The samples were thawed and clarified by centrifugation at $16,000 \mathrm{Xg}$ for 5 minutes, and equal cell volumes of the nanoluciferase samples were processed using the Nano-Glo nanoluciferase assay system (Promega) according to the manufacturer's 
instructions. Luminescence was then recorded using a Synergy H1 microplate reader (BioTek).

\section{Statistical Analysis}

The quantitative data reported in this study are the means of a minimum of three independent biological replicates, unless specifically noted otherwise. The growth curve data presented in Fig. 2.2 were statistically assessed using an area under the curve approach to determine differences in viral growth kinetics throughout the course of the assay. The statistical analysis of comparative samples was performed using variable bootstrapping where appropriate, as previously described [137]. The error bars indicate the standard deviation of the mean. The $P$ values associated with individual quantitative data sets are the result of Student's $t$ test for the corresponding quantitative data.

\section{Results}

\section{SINV vRNA Capping Can be Modulated by Point Mutations in the nsP1 Protein}

As described above, we recently reported that a significant number of SINV genomic vRNAs produced during infection lack the type-0 5' cap structure. Despite being noninfectious, the noncapped viral genomic RNAs (ncgRNAs) are produced in a temporally dependent manner and are packaged into viral particles throughout the course of infection [99]. Given the evolutionary conservation of the production of the ncgRNAs during alphaviral infection, we hypothesized that 
they must be biologically important to viral infection. This led us to question how modulating capping activity, which would alter the production of capped viral genomic RNAs and ncgRNAs, impacts viral infection. In order to determine the biological importance of the ncgRNAs during viral infection, we modulated the methyltransferase and guanylyltransferase activities of the alphaviral nsP1 protein to generate mutant SINV strains with either increased or decreased rates of viral capping. These mutations were based on previous work done in VEEV by Li et al. (2015), where alanine substitutions at specific residues affected the nsP1 protein's methyltransferase and guanylyltransferase activities, as well as cap formation as a whole. Specifically, the previous study found that alanine substitutions at Y286 and N376 decreased RNA capping efficiency, although to different extents, whereas an alanine substitution at D355 increased RNA cap formation. All three residues are highly conserved across multiple alphaviruses, including the model alphavirus SINV (Fig. 2.1A). Modeling of the SINV nsP1 protein using ITASSER indicates that residues D355 and N376 are likely close to one another, in parallel alpha helices proximal to the catalytic site within the Iceberg Domain (Fig. 2.1B)[146]. Nonetheless, Y286 is located at a distal site far from the putative active sites, as identified by prior mutational analyses $[35,132$, 133]. Admittedly, the ITASSER predicted structure is unlikely to be a wholly accurate representation of the true structure of the SINV nsP1 protein. Despite the inherent inaccuracy of protein folding prediction algorithms in the absence of a closely related crystal structure, secondary and tertiary structural elements reminiscent of methyltransferase and guanylyltransferase enzymes may be 
detected, including a Rossmann fold-like structure surrounding the key residues involved in methyltransferase activity $[35,147]$. In addition, the overall arrangement of the Core Region and Iceberg Domains are largely intact, and the known membrane anchoring domains face outward from the globular body of the protein [147]. The differential ability of the Y286, D355, and N376 to affect VEEV nsP1 capping activity as well as their locations in highly conserved domains, which play integral roles in cap formation, made them strong candidates for testing how modulating the capping activity of nsP1 affects SINV infection.

In order to verify that the SINV nsP1 mutations altered the rate of vRNA capping, we assessed what proportion of viral particles contained capped RNA genomes. We have shown previously that both capped genomic RNAs and ncgRNAs are packaged and released during viral infection [99]. Thus, knowing what proportion of capped viral RNA is being packaged into viral particles gives insight into the general capping efficiency of nsP1 during viral infection. To this end, the comparative assessment of viral capping was accomplished by comparing the relative sensitivity of the mutants to RNAse degradation using XRN-1, a 5'-3' exoribonuclease which preferentially degrades RNAs lacking a protective 5' cap structure, in particular 5' monophosphate RNAs [139, 140]. By measuring the amount of RNA resistant to XRN-1 degradation, we were able to determine what relative proportion of the total input RNA was capped relative to wild type SINV, as the predominant noncapped 5' end was previously determined to be a 5' monophosphate [99]. As shown in Figure 2.1C, both SINV N376A and SINV Y286A had significantly lower proportions of $X R N-1$ resistant genomic 
RNAs compared to WT, with SINV Y286A having approximately $25 \%$ less XRN-1 resistant RNA compared to WT, and SINV N376A having approximately $75 \%$ less $\mathrm{XRN}-1$ resistant RNA. In contrast, and as expected from the previous study by Li et al. (2015), SINV D355A was found to have a significantly greater proportion of XRN1-resistant RNA, with a mean approximately four-fold greater than what was observed for WT SINV. To confirm that the observed resistance of the SINV D355A genomic RNAs was due to a cap structure, the genomic RNAs were subjected to XRN-1 nuclease treatment in the presence of an established decapping enzyme $[99,142,143]$. As shown in Figure 2.1D, enzymatic removal of the 5' cap structure eliminated the resistance of the D355A derived genomic RNAs to XRN-1 treatment, resulting in XRN-1 resistances comparable to WT SINV.

From these data, we were able to conclude that the point mutations engineered into the SINV nsP1 protein can indeed alter capping activity, both negatively, in the case of Y286A and N376A; and positively, as with D355A. Therefore, the individual nsP1 mutants can be used to assess the impact of the ncgRNAs by modulating the capping activity of nsP1 in tissue culture models of infection in a controlled manner.

\section{Increased Capping Decreases SINV Growth Kinetics in Mammalian Cells}

While the effects of synonymous point mutations on nsP1 capping efficiency have been previously characterized for VEEV at an enzymatic level [124], the effects of modulating capping activity on viral infection as a whole has 
not yet been studied in detail. Given the molecular function of the 5' cap structure, one could expect that the SINV nsP1 mutants that decreased vRNA capping would show impaired viral growth kinetics relative to wild type virus. Likewise, if the ncgRNAs were truly nonfunctional, a mutant that increased capping would show enhanced viral growth kinetics in regards to wild type infection. As demonstrated by the data presented in Fig. 2.2A, decreasing SINV capping modestly decreased viral growth kinetics, as observed for the SINV Y286A and SINV N376A mutants. However, the SINV D355A mutant, which increased vRNA capping relative to WT, exhibited significantly decreased titers over the course of infection (Fig. 2.2A). In addition to a 2-log decrease in viral titer, the SINV D355A mutant produced plaques approximately half the size of those produced by wild type SINV (Fig. 2.2B and 2.2C). Despite not showing significantly decreased viral growth kinetics, both the SINV Y286A and SINV N376A mutants also exhibited a small plaque phenotype, albeit not to the same extent as the SINV D355A mutant. In addition, all three capping mutants were found to have decreased induction of cell death compared to WT SINV (Fig. 2.2D).

Collectively, these data indicate that changing the efficiency of vRNA capping negatively impacted viral growth kinetics, illustrating that a step in the viral life cycle has been detrimentally affected. Furthermore, the viral growth kinetics data suggests that SINV is more sensitive to increased vRNA capping efficiency than it is to decreased capping efficiency. Thus, the ncgRNAs produced during infection are indeed biologically important. Nevertheless, 
despite the clear negative impact to viral infection, the precise nature of the molecular consequences of increased vRNA capping and decreased presence of ncgRNAs cannot be determined from these data alone. As such, the viral gene expression and vRNA synthesis profiles of each of the SINV nsP1 mutants were next assessed to determine if they were negatively affected by modulation of vRNA capping.

\section{Translation of the SINV Genomic RNA Correlates with Capping Efficiency}

For the majority of mRNAs, a key factor for determining whether or not an mRNA is translated is the presence of a functional 5' cap structure [148-150]. Therefore, changing the ncgRNAs to capped genomic RNAs or vice versa by altering viral capping efficiency should impact the amount of protein being produced by viral RNA during infection. To investigate the effect(s) that modulating nsP1 capping efficiency has on viral gene expression, a SINV reporter, which contained the open reading frame of nanoluciferase in frame with the nsP3 nonstructural protein, was used to measure viral genomic RNA translation throughout infection (Fig. 2.3A) [99]. Nanoluciferase expression was measured at regular intervals over the course of viral infection (Fig. 2.3B). As expected, the SINV D355A mutant, which increased capping relative to wild type, exhibited increased genomic VRNA translation compared to WT SINV for every time point measured (Fig. 2.3C). Similarly, SINV N376A, which decreased capping relative to wild type, showed decreased translation (Fig. 2.3C). Curiously, the SINV Y286A mutant showed differential nanoluciferase expression 
during infection, with translation being slightly increased very early during infection, decreased at 8hpi, and similar to wild type at later times post infection.

While the SINV nanoluciferase reporter virus allows for the accurate quantification of viral gene expression during infection, it does not measure the sum accumulation of the nonstructural proteins. To measure the relative accumulations of the viral nonstructural proteins, the levels of SINV nsP2 protein were assessed via Western blotting at 8, 12 and 16hpi. As shown in Fig. 2.4A and quantified in. Fig 2.4B, at $8 \mathrm{hpi}$ the accumulation of the SINV nsP2 proteins between wild type SINV, SINV D355A, and SINV N376A differed to a statistically significant extent. The abundance of nsP2 was increased $\sim 2.5$-fold during SINV D355A infection, relative to wild type SINV. In contrast, nsP2 levels during SINV N376A infections were decreased $\sim 2$-fold relative to wild type SINV infections. The SINV Y86A mutant was more or less equivalent to wild type nsP2 levels. Similar trends were observed at later times during infection. As depicted in Figs. 2.4C and $2.4 \mathrm{D}$, at $12 \mathrm{hpi}$ the SINV nsP2 levels were statistically increased relative to wild type infection; however, given the low magnitude of effect, these differences are unlikely to be biologically significant. In contrast, at $16 \mathrm{hpi}$ SINV $\mathrm{nsP} 2$ levels were increased to an extent that is likely biologically meaningful (Figs 2.4E and 2.4F).

Overall, the levels of genomic translation for the SINV nsP1 mutants nicely reflect their relative differences in capping efficiency, with increased vRNA capping resulting in increased translation of the nonstructural proteins, and decreased vRNA capping resulting in decreased translation at 8hpi. These data 
are consistent with the conversion of the ncgRNAs to translationally competent capped genomic vRNAs. Furthermore, western blotting confirmed the differences in translational activity detected during SINV nanoluciferase reporter infections. Taken together, the nanoluciferase and western blot data indicate that the translation of the genomic vRNAs continues during infection with SINV D355A beyond what is observed for wild type SINV. Nonetheless, the biological differences in translational activity observed between the SINV D355A mutant and its respective parental wild type strain fail to explain the approximately 200fold reduction in viral titer.

\section{Modulating SINV Capping Does Not Alter Overall RNA Synthesis or Accumulation}

Since differences in viral gene expression failed to outright explain the observed decreased viral titers associated with the SINV D355A mutant, we next sought to identify if vRNA synthesis was impaired as a result of nsP1 mutation. To determine what impact altering viral capping efficiency has on vRNA synthesis, the genomic, subgenomic, and minus strand vRNAs were quantitatively assessed at 2, 4, and 8 hours post infection via qRT-PCR. At $2 \mathrm{hpi}$, the SINV D355A mutant, which has increased vRNA capping relative to wild type SINV, produced slightly more SINV genomic and subgenomic RNAs; whereas the SINV N376A mutant, which exhibits decreased vRNA capping, produced slightly fewer genomic and subgenomic RNAs (Fig. 2.5A). In general, the differences in the amount of genomic and subgenomic RNAs being produced by 
these two capping mutants is reflective of the differences observed in the synthesis of their replication machinery (Figs. 2.3 and 2.4). At $4 \mathrm{hpi}$, all three SINV nsP1 mutants show a statistically significant deficit in the amount of subgenomic RNA present (Fig. 2.5B). Nonetheless, these deficits are unlikely to be biologically meaningful due to their overall magnitude of effect relative to wild type SINV. By 8hpi however, all three capping mutants show similar levels of all three RNA species compared to WT, with the exception of N376A, which had less minus strand template compared to WT (Fig. 2.5C).

Altogether, these data demonstrate that altering the capping activity of nsP1 may have impacts on RNA synthesis very early during viral infection, but that these differences become muted as infection progresses. Importantly, these data indicate that vRNA synthesis on the whole has not been disrupted, precluding the possibility that the point mutations made in nsP1 have disrupted the function of the other nonstructural proteins. Moreover, similar to that described above regarding viral gene expression in the previous section, the $\sim 2$ log reduction in viral growth kinetics observed for the SINV D355A mutant is not due to decreased VRNA accumulation or defective vRNA synthesis.

\section{Translation of the SINV Subgenomic RNA is Largely Unaffected by Viral Capping}

Given that neither the differences in nonstructural gene expression or vRNA synthesis were capable of explaining the negative impact of the SINV nsP1 D355A mutation, we next sought to determine if the function of the 
subgenomic vRNAs were impacted. As both the genomic and subgenomic vRNAs are capped by nsP1, it was hypothesized that mutations which increased capping of the genomic vRNAs might impact subgenomic vRNA function. As such, it could be expected that these vRNAs would exhibit similar responses to altered capping in terms of translation. In order to determine whether translation of the subgenomic vRNAs were impacted similarly to the genomic vRNAs, the amount of protein produced late during infection was measured using metabolic labeling (Fig. 2.6A). Curiously, both the SINV D355A and SINV N376A mutants exhibited a modest decrease in viral capsid production (Fig. 2.6B). In addition, none of the SINV nsP1 mutants exhibited differences in regards to the shutoff of host translation, a hallmark of alphaviral infection as reviewed in [151], as evidenced by the labeling of the cellular Actin protein, relative to WT SINV (Fig. 2.6C). Interestingly, however, the ongoing synthesis of a high molecular weight protein consistent with the nonstructural polyprotein was reproducibly detected during the metabolic labeling of SINV D355A mutant infections.

Collectively, these data indicate that changes in capping efficiency of the genome may either not lead to equivalent changes in capping of the subgenomic VRNA; or alternatively, that SINV, and likely other alphaviruses, regulate the translation of the structural proteins differently than the nonstructural proteins [152]. Regardless, these data suggest that subgenomic translation and host translational shutoff are not impacted to a biologically significant degree by modulating the efficiency of vRNA capping, and thus are not responsible for the decreased viral titer associated with increased capping efficiency. 


\section{Increasing SINV Capping Decreases Viral Particle Production}

Given that the molecular characterizations of the SINV nsP1 mutants had so far failed to identify the molecular defect leading to decreased viral growth kinetics, we expanded our analyses beyond the lifecycle events most obviously affected by the 5' cap structure. Since vRNA synthesis and viral gene expression were unaffected, we next sought to determine whether viral particle assembly was negatively impacted by increasing the capping efficiency of the SINV nsP1 protein.

To determine whether increasing capping efficiency affected viral particle production, we quantified the total number of particles produced by each mutant as well as wild type SINV after $24 \mathrm{hrs}$ of infection. Similar to what was observed during the kinetic analyses of viral infection, increased vRNA capping was associated with the production of significantly fewer particles, with the SINV D355A mutant producing 25-fold fewer particles (as measured by genome equivalents per $\mathrm{ml}$ ) than wild type SINV (Fig. 2.7A). In contrast, particle production was largely unaffected during infections of the SINV Y286A and SINV N376A mutants, which decreased vRNA capping.

The point mutants utilized in this study are located within, or closely adjacent to, the SINV packaging signal [153]. Previous characterizations of the alphaviral packaging signals have indicated the importance of stem-loop structures which contained a guanosine triplet in the loop region. Mutational analyses of the alphaviral packaging elements determined that mutation of the 
guanosine triplets, or deletion of the packaging element altogether, significantly reduced viral titer due to nonselective particle assembly leading to the production of alphaviral particles containing the subgenomic vRNAs [153]. Further experiments defined that a minimum of two guanosine triplet stem loops was sufficient to impart wild type particle production with selectivity for the genomic RNA. Even though none of the point mutants used in this study impact either the guanosine triplets, or the general secondary structures (as predicted by in silico analysis), the potential for the inhibition of assembly by a non-capping mechanism existed, warranting further assessment of particle production during SINV nsP1 mutant infections. As mentioned earlier, disruption of the alphaviral packaging signal leads to the production of viral particles containing predominantly subgenomic RNAs [153]. As shown in Fig. 2.7B, quantitative determinations to identify which specific vRNAs were packaged into wild type and SINV nsP1 mutant viral particles indicated no aberrant packaging of the SINV subgenomic RNA, consistent with an intact functional packaging signal.

The high degree of similarity between the relative magnitude of effect regarding decreased viral titer and viral particle production during SINV D355A infection indicates that particle assembly, and not the slight perturbations in vRNA synthesis or structural gene expression, is primarily responsible for decreased viral growth kinetics. Importantly, these data suggest that SINV, on the whole, is much more tolerant of mutations which decrease capping efficiency than those that increase capping efficiency, and that by increasing the capped to noncapped viral RNA ratio, viral particle production has been detrimentally 
affected. Moreover, these data validate that the packaging signal remains functional in the presence of the nsP1 point mutations described in this study.

\section{Discussion}

\section{The capping of SINV genomic vRNAs can be modulated by point mutations in nsP1}

The data shown in Fig. 2.1 indicates that, in SINV, 5' capping of the genomic vRNA can be modulated via single point mutations in the nsP1 protein. This is true for both increasing vRNA capping, as seen with SINV D355A, and decreasing capping, as seen with SINV Y286A and SINV N376A. Moreover, vRNA capping can also be modulated to different extents, as seen with SINV Y286A and SINV N376A, which decreased vRNA capping by $25 \%$ and $75 \%$, respectively. The ability to change nsP1 capping efficiency in a controllable manner opened up new avenues to explore the molecular and biological importance of both the capped genomic vRNAs and the ncgRNAs during infection in tissue culture models of infection and in vivo.

From the data above, we may conclude that a primary consequence of mutating the SINV nsP1 protein is the alteration of vRNA capping. Nonetheless, the alphaviral nonstructural proteins interact with one another during infection [154-157]. Previous studies have shown that disrupting these interactions by mutation results in poorer viral infection and, more specifically, leads to severe defects in vRNA synthesis [154-156]. However, the disruption of nonstructural protein interactions is unlikely with the nsP1 mutants reported in this study, as 
the phenotypes described for situations where the interactions between the nonstructural proteins have been disrupted are inconsistent with what is reported here. For example, several residues in nsP4 have been reported as important for interactions with nsP1, such as G38L in nsP4 [154]. When this residue in nsP4 was mutated, viral infection exhibited decreased growth kinetics and a small plaque phenotype. However, a hallmark of disrupting the nonstructural protein interactions was the severely decreased synthesis of minus strand vRNA throughout the course of infection $[154,156]$. As seen in Fig. 2.5, none of the nsP1 mutants examined in this study exhibited a significant deficit in production of any viral RNA species, with the exception of a minor decrease in minus strand vRNA synthesis by N376A. It is of note that the nsP4 G38L mutant was able to be rescued by an additional mutation in nsP1 at N374 [154]. The nsP1 N374 mutation resulted in complete restoration of viral titer and partial restoration of minus strand vRNA synthesis in the nsP4 G83L background compared to wild type. While the effect of mutating nsP1 N374 on capping efficiency is not known, one could speculate that, given its proximity to other residues which we have shown alter capping activity, that modulating the capping efficiency of nsP1 could be a way of coping with the detrimental nsP4 G38L mutation, which creates a severe defect in RNA synthesis. In addition to this, a previous study has reported that the region in nsP1 encompassing the SINV point mutations utilized here possess little to no interaction with the nsP2 protein [157]. Thus, for the reasons described above, the nsP1 residues mutated during this study are likely not involved in mediating nonstructural protein interactions, and the resulting 
deficits in the viral lifecycle are not due to disrupted nonstructural protein interactions.

\section{SINV infection is more sensitive to increased capping than decreased capping}

Multiple studies have shown that polymorphisms in nsP1 have profound effects on virulence. Mutations in regions of nsP1 have been shown to alter vRNA synthesis, viral titer, viral sensitivity to IFN, and disease severity in vivo $[97,154,158]$. Certain residues, such as nsP1 H39 in SINV, completely abrogate viral infection by eliminating the methyltransferase activity of the nsP1 protein $[35,134,159]$. Therefore, we expected that incorporating the point mutations which alter viral capping would have impacts on viral infection. However, we were surprised to find that while the increasing capping mutant was found to have decreased viral growth kinetics, the decreased capping mutants were not significantly different from wild type SINV (Fig. 2.2A). This result was especially surprising when it was found that all three capping mutants had small plaque phenotypes and decreased cell death, yet D355A was the only mutant to show altered growth kinetics (Fig. 2.2B, C, and D). Serial passaging of the mutants used in this study have indicated that they are stable for at least 4 sub-passages, as no reversion events (based on plaque phenotype) were observed for any of the SINV nsP1 mutants. This suggests that SINV is more detrimentally impacted by changes which increase the amount of capped vRNA present than those which decrease capping efficiency. Thus, increasing the capping efficiency of the 
SINV nsP1 protein, which effectively reduced the production of the ncgRNAs, indicates that the ncgRNAs are biologically important to viral infection.

\section{Altering capping leads to changes in genomic translation but not RNA synthesis or subgenomic translation}

As would be expected, changes in nsP1 capping efficiency correlated with changes in genomic vRNA translation. In addition, the reduced presence of the ncgRNAs correlated with increased translation of the viral genomic RNA throughout infection, as can be seen with the increased capping mutant SINV D355A. Furthermore, decreasing the capping efficiency of the nsP1 protein, as evidenced by the SINV N376A mutant, modestly decreased translation. Admittedly, the second decreased capping mutant, SINV Y286A, did not follow the same pattern as the SINV N376A mutant. This may be due to the comparatively minor decrease in capping efficiency caused by SINV Y286A not being significant enough to consistently alter the vRNA population leading to dysregulated genomic translation.

However, the increased translation exhibited by the SINV D355A mutant did not lead to lasting compounding biological effects, at least in tissue culture models of infection. For instance, despite there being more replication machinery being produced early during SINV D355A infection, there were no overt differences in the synthesis of any of the viral RNA species during the time points tested (Fig. 2.5). This suggests that increasing or decreasing the production of the nonstructural proteins alone is not enough to alter RNA synthesis over the 
long term in highly permissive tissue culture models of infection. Nonetheless, due to technical limitations we were unable to accurately assess vRNA synthesis earlier than two hours post infection. Hence, the possibility that vRNA synthesis is enhanced very early during infection remains unaddressed.

Another unexpected result was that changes in capping efficiency seems to affect subgenomic translation differently than genomic translation. The data presented in Fig. 2.6 show that both the increased and decreased capping mutants SINV D355A and SINV N376A demonstrate slight, biologically unmeaningful, decreases in capsid production at 16hpi, despite having notable differences in nonstructural gene expression (Figs. 2.3 and 2.4). This suggests that either capping efficiency is regulated differently for the subgenomic RNA than for the genomic RNA, resulting in no differences in capping efficiency for the subgenomic RNA, or that translation of the subgenomic RNA is less dependent on the presence of a 5' cap than the genomic RNA during infection. The latter is supported by previous studies which demonstrate that the elF4F complex, which includes the cap-binding elF4E, is not needed in order to initiate translation of the alphaviral subgenomic RNA $[152,160]$. These previous studies, along with the data presented here, suggests that translation of the subgenomic RNA is unaffected, at least in part, by modulation of vRNA capping.

In addition to there being little effect on subgenomic translation, there was also shown to be no differences in terms of host translational shutoff between any of the capping mutants and WT SINV (Fig. 2.6C). This further supports that the point mutations made in nsP1 are not negatively affecting interactions 
between nonstructural proteins, because disruptions between nsP1 and nsP4 have been previously found to negatively affect host translational shutoff [154]. Host translational shutoff, for SINV at least, has been largely attributed to the translation of the structural proteins [161]. Therefore, it is unsurprising that there is no change in host shutoff between the capping mutants and the WT SINV given that there is little difference in subgenomic expression.

\section{Decreased titer due to changes in capping caused by interference with particle production}

The decreased viral growth kinetics observed during SINV D355A infection correlated remarkably with decreased particle production. However, the congruence of the decrease in infectious units and viral particles infers that viral infectivity is, more or less, identical for all of the viral strains. The discrepancy between the two magnitudes of effect (an approximate 5-fold difference) are likely due to confounding variations in the accuracy and precision of the two measurements. Nonetheless, it remains possible that the viral particles have differences in their basal infectivity. Studies examining earlier effects of the ncgRNAs are ongoing and will be presented in a follow up study.

The observation that decreased particle production is the primary molecular defect during SINV D355A infections suggests that increasing the amount of capped genomic RNAs, thereby decreasing the number of ncgRNAs, negatively impacts the assembly of nascent viral particles. Whether or not the increased capping activity is directly, or indirectly, responsible for the packaging 
phenotype isn't definitively known. Characterizations of SINV packaging indicates selectivity remains intact during the SINV D355A assembly process despite decreased particle production overall, as the packaging of subgenomic vRNAs was not observed. This is indicative of a functional alphaviral packaging signal despite the incorporation of minor point mutants into the region defined as the packaging signal for SINV. Moreover, the SINV Y286A mutant which also resides within the SINV packaging element lacks an appreciable packaging phenotype. Thus, the assembly phenomena associated with the SINV D355A mutant cannot be simply explained by disruption of the alphaviral packaging signal.

While the underlying mechanism is unclear, the data presented above indicates that nonstructural protein expression is increased relative to wild type parental virus, and remains increased well into the late stages of infection during SINV D355A infections. Collectively, these data indicate that decreasing the production of the ncgRNAs perturbs viral genomic RNA function beyond the individual RNA level, as apparent compounding effects on the genomic RNA population are observed. Precisely how the translationally inactive ncgRNAs serve to modulate genomic vRNA function as a whole, leading to efficient particle assembly, is unknown.

We propose that, as diagrammed in Fig. 2.8, during wild type infections, the translationally inactive, ncgRNAs temper the molecular activities of the translationally active, capped genomic vRNA population allowing for the temporal progression of infection to lead to the assembly and release of viral particles. We postulate that the ncgRNAs, due to their lack of translational capacity, interact 
with a unique set of host factors relative to the capped translationally competent genomic RNAs. Collectively, these interactions lead to the development of a proassembly microenvironment by excluding host factors that either inhibit the assembly process, or promote nucleocapsid disassembly. For instance, if the ncgRNAs foster an non-translational environment through the interaction of host factors, such as those found within stress granules, the 605 ribosomal subunit, which is implicated in nucleocapsid disassembly would be excluded from the local microenvironment, allowing assembly to occur unimpeded [26, 162]. However, when the genomic RNA population is altered by increasing the efficiency of genomic RNA capping, such as observed with the SINV D355A mutant, the increased and continuous translational activity of the genomic vRNA culminates in the formation of a pro-translational vRNA "pool" that is refractory to encapsidation and particle assembly. Work examining such possibilities are ongoing in the Sokoloski lab and will be reported in the future.

\section{Conclusions}

Collectively these data affirm the existence and biological importance of the ncgRNAs during SINV infection. This assertion is directly supported by the capacity to modulate the capping activities of nsP1 protein via site directed mutagenesis resulting in the increased production of $X R N-1$ resistant genomic vRNAs without increased overall genomic RNA numbers. Moreover, the preponderance of gene expression data indicating increased translation brought about by increasing the capping of the genomic vRNA supports the existence of 
the alphaviral ncgRNAs at a functional level. Finally, the molecular characterizations of SINV nsP1 mutant infections provides insight into the biological importance of the ncgRNAs in regards to the regulation of alphaviral infection at the molecular level. 


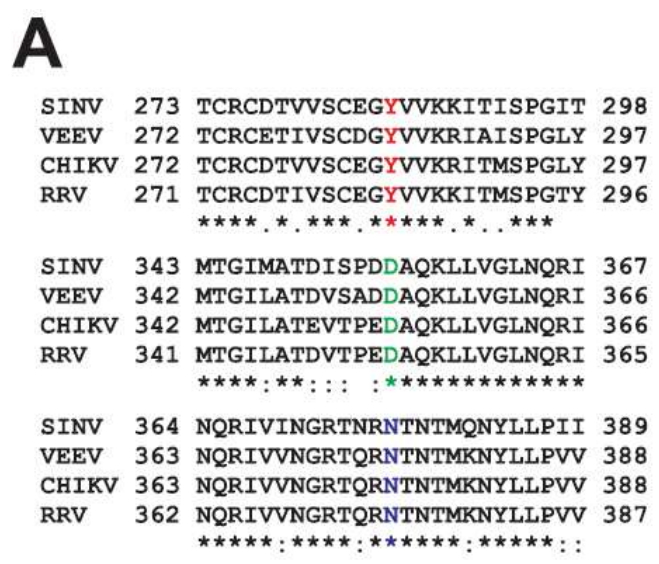

C

Relative Quantity of XRN-1 Resistant Genomic RNA

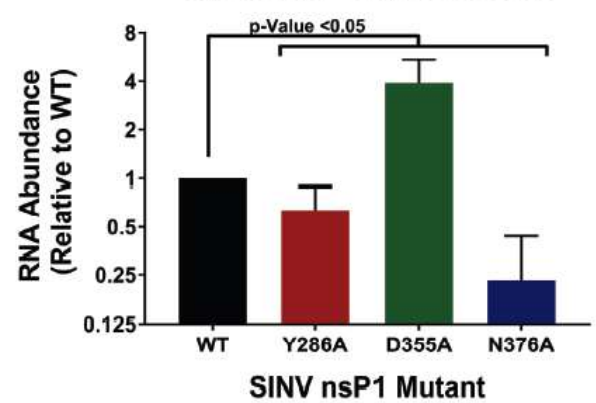

B

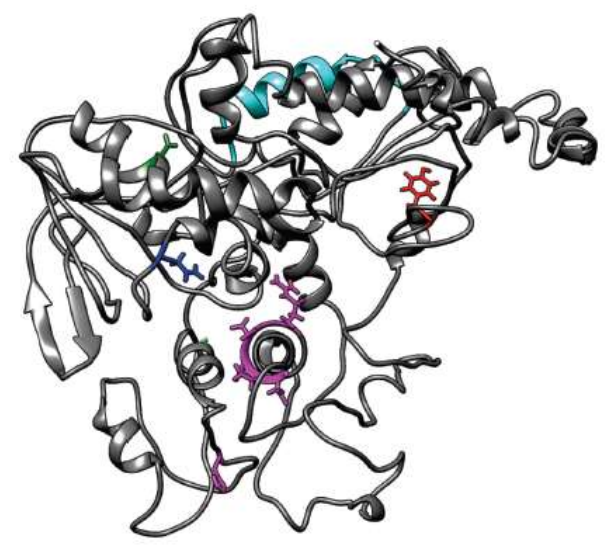

D

Relative Quantity of XRN-1 Resistant Genomic RNA After Decapping

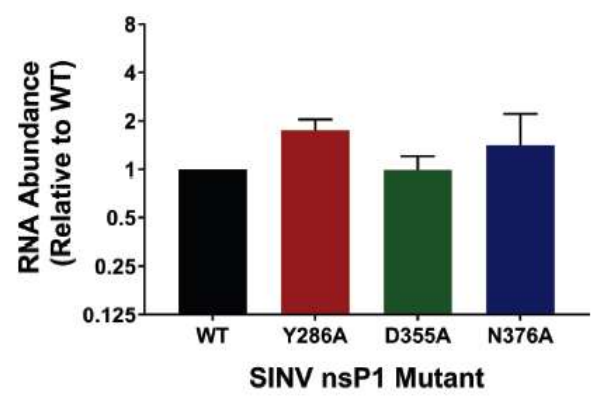

Figure 2.1. Point mutations in the nsP1 protein of SINV alter 5' vRNA

capping efficiency. (A) Amino acid sequence alignment of selected alphavirus

nsP1 proteins. The individual nsP1 protein sequences of Sindbis (ViPR-U38305),

Venezuelan Equine Encephalitis (VEEV, ViPR-L01443), Chikungunya virus

(CHIKV, ViPR-DQ443544), and Ross River virus (RRV, ViPR-GQ433359) were

aligned by Clustal Omega. (B) An ITASSER predicted structure of the SINV nsP1

protein. Amino acid residues of importance are highlighted as follows-

Red $=Y 286$, Green=D355, Blue=N376, Cyan=amphipathic helix, and the residues

involved in the methyltransferase activities, including $\mathrm{H} 39$ which binds to the

${ }^{\mathrm{m}} \mathrm{GMP}$ residue are pink. (C) A graph depicting the relative quantity of $\mathrm{XRN}-1$

resistant vRNA isolated from viral particles produced by $\mathrm{BKH}-21$ cells infected 
with wild-type SINV or the individual capping mutants $24 \mathrm{hpi}$. (D) Identical to panel C, with the exception that the viral genomic RNAs were enzymatically decapped concurrent with $\mathrm{XRN}-1$ treatment. All quantitative data shown represents the means of a minimum of three independent biological replicates utilizing 3 independent particle preparations, with the error bars indicating the standard deviation of the means. P-Values, as indicated on the figure, were determined by Student's T-test. 

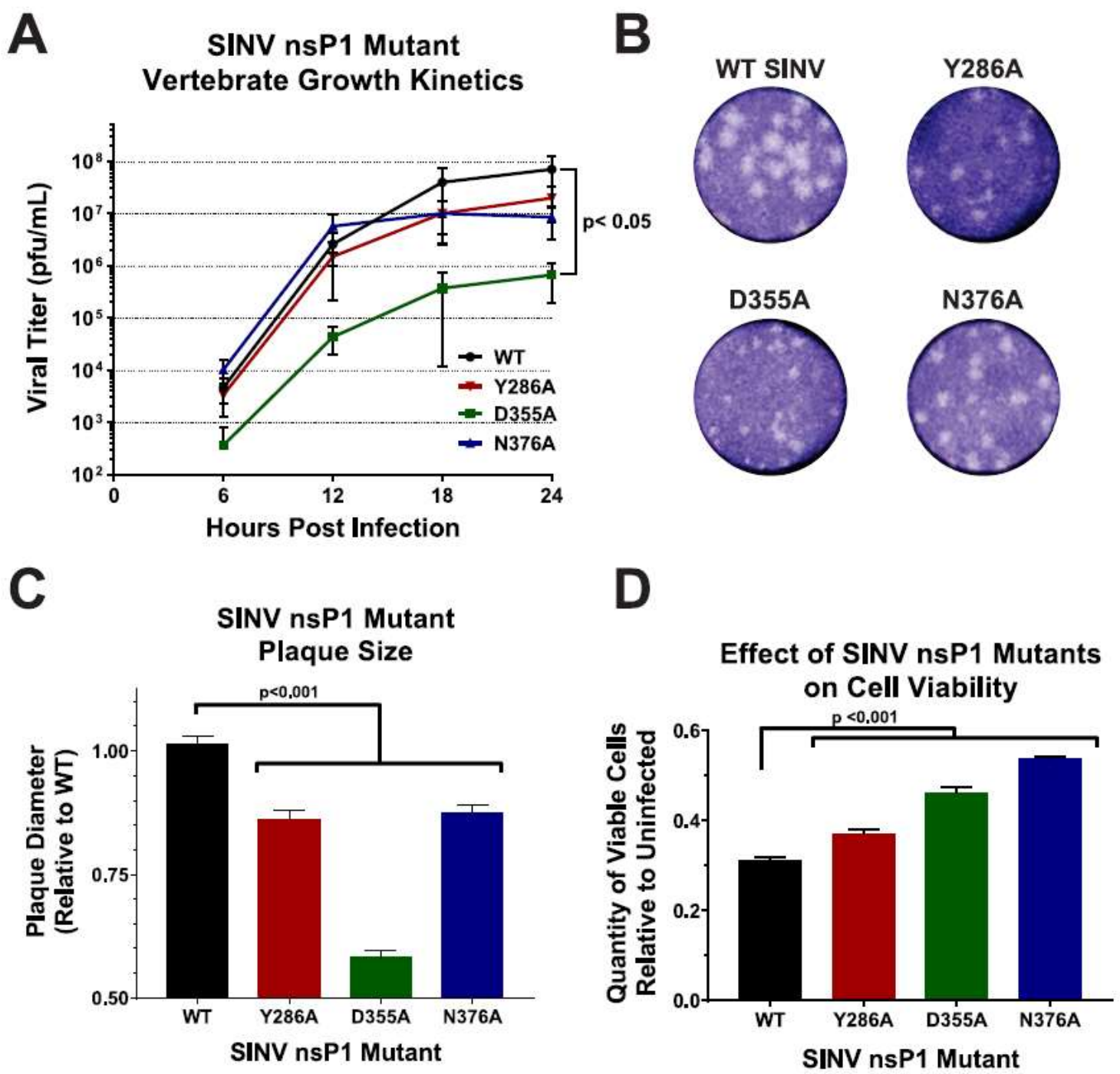

Figure 2.2. Altering viral capping efficiency negatively impacts viral infection. (A) One-step growth kinetics of the individual capping mutants and parental wild type SINV as observed in BHK-21 cells infected at an MOI of 0.5 PFU/cell. Statistical significance was determined by area under the curve analysis. (B) Plaque morphology of wild type SINV and capping mutant viruses in BHK-21 cells overlaid with $10.5 \%$ solution of Avicel at $24 \mathrm{~h}$ post-infection. (C) A graph indicating the average plaque diameter of the mutant viruses relative to 
wild type SINV. Size was determined by ImageJ software (NIH). (D) Cell viability of BHK-21 cells infected with the individual capping mutants at 24hpi relative to mock infected BHK-21 cells. All quantitative data shown represent the means of at least three independent biological replicates, with the error bars representing standard deviations of the mean. Statistical significance, as indicated within each panel, was determined by Student's $t$ test. 


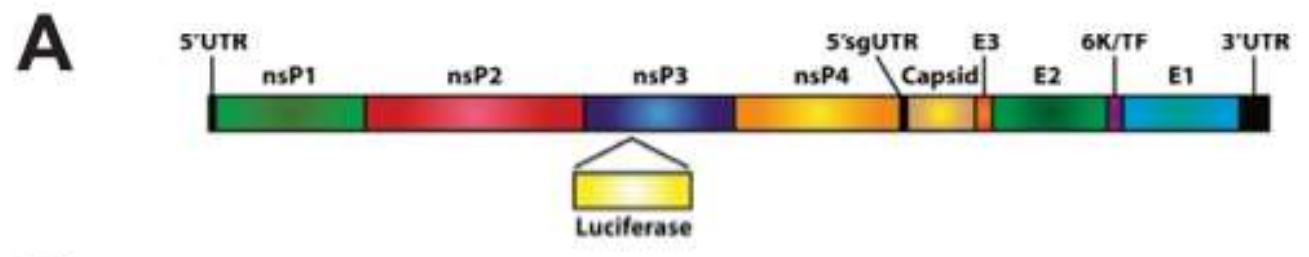

B

\section{Translation of the SINV Genomic RNA}

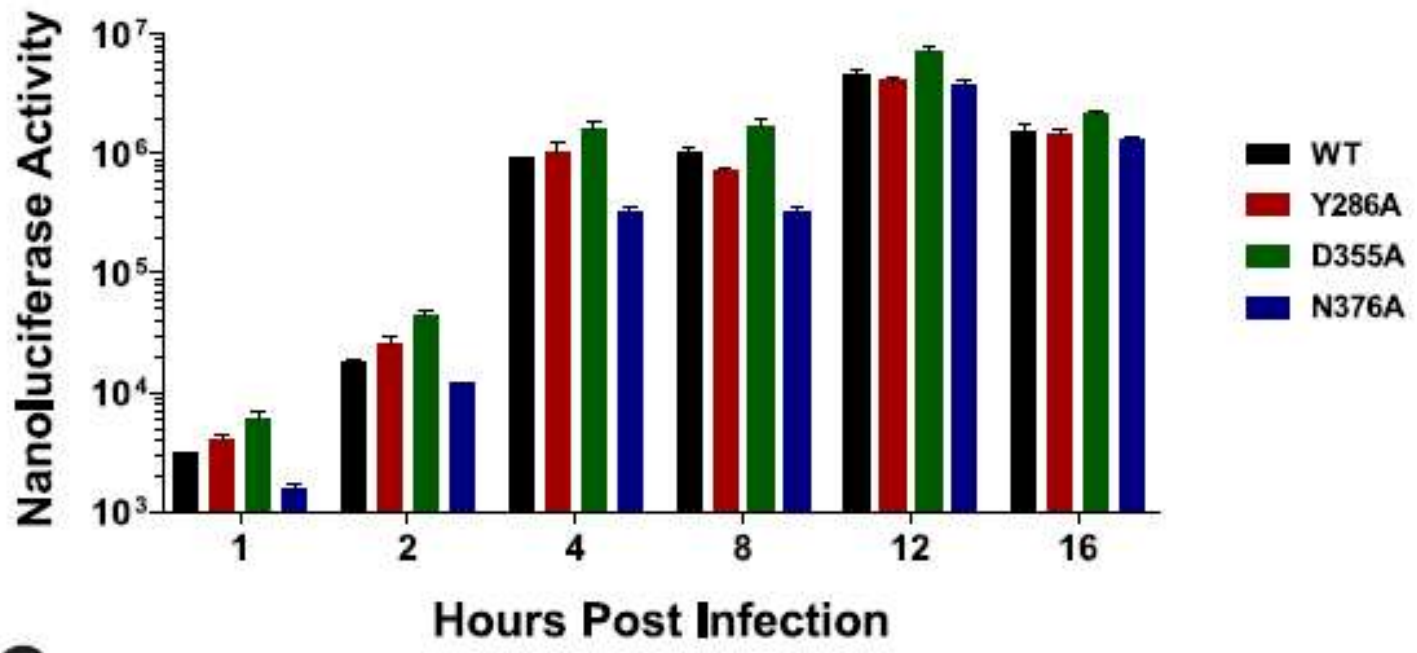

C

Relative Translation of the SINV Genomic RNA

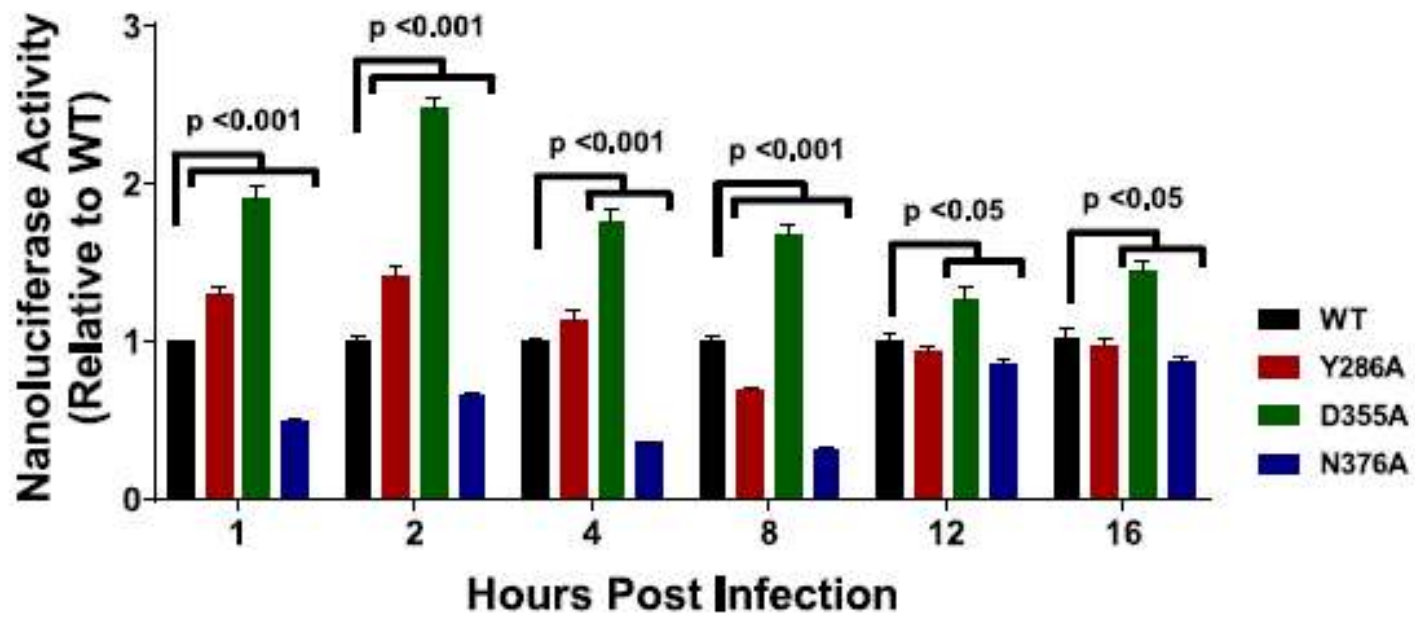

Figure 2.3. Translation of the genomic vRNA correlates with viral capping efficiency. (A) Schematic diagram of the SINV nanoluciferase reporter used in this study. (B) BHK-21 cells were infected with either parental wild type, or an 
individual SINV capping mutant nanoluciferase reporter strain. The level of nanoluciferase activity was quantified at the indicated times post infection. C) The nanoluciferase activity, as reported in panel B, normalized to wild type expression at each individual time point to enable readers to identify differences in translation. All the quantitative data shown represent the means of three independent biological replicates, with the error bars representing standard deviations of the mean. Statistical significances, as indicated in the figure, were first determined using ANOVA analyses followed by post-hoc statistical analyses by Student's $t$ test. 

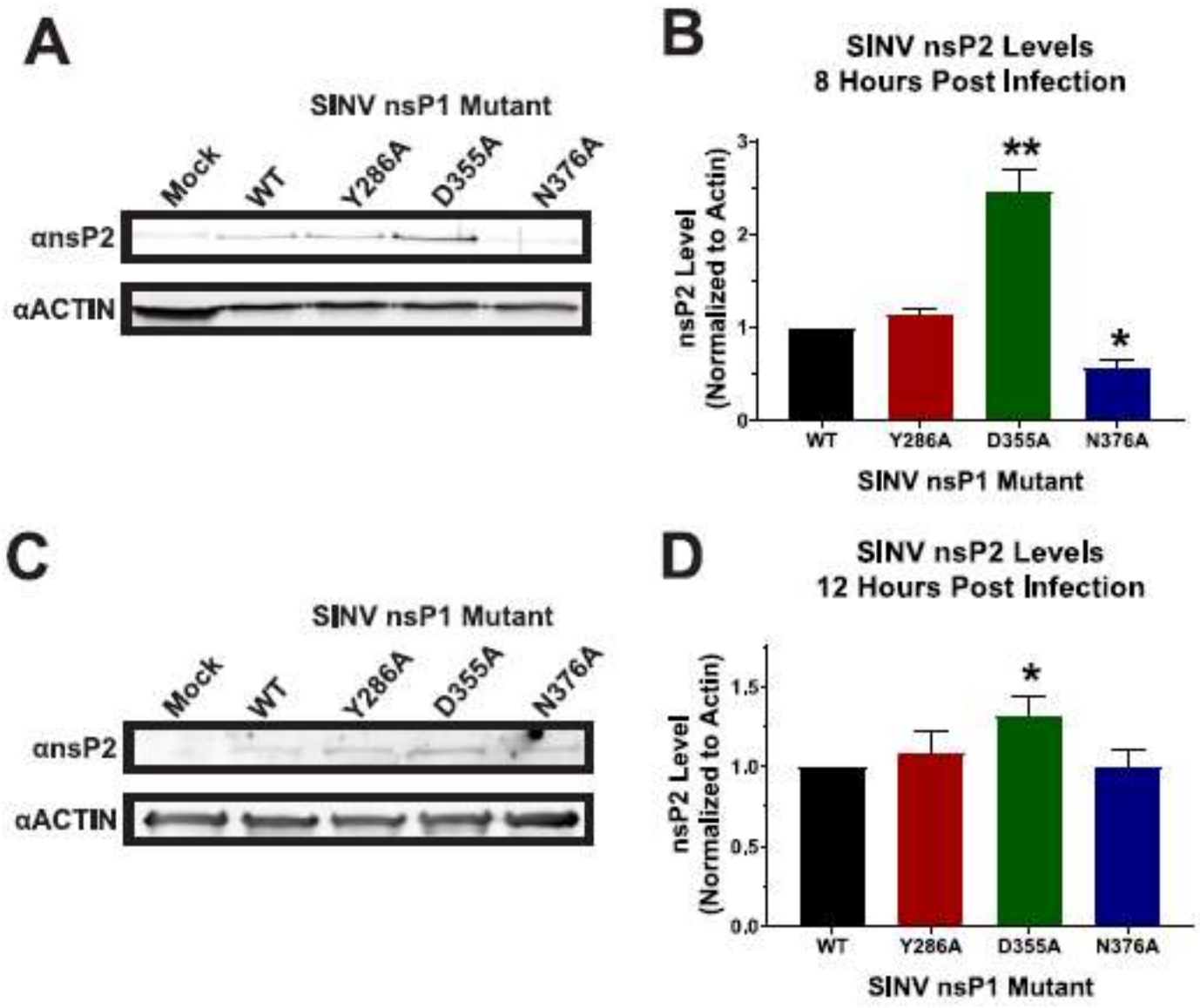

$\mathbf{E}$

$F$

SINV nsP2 Levels 16 Hours Post Infection

SINV nsP1 Mutant
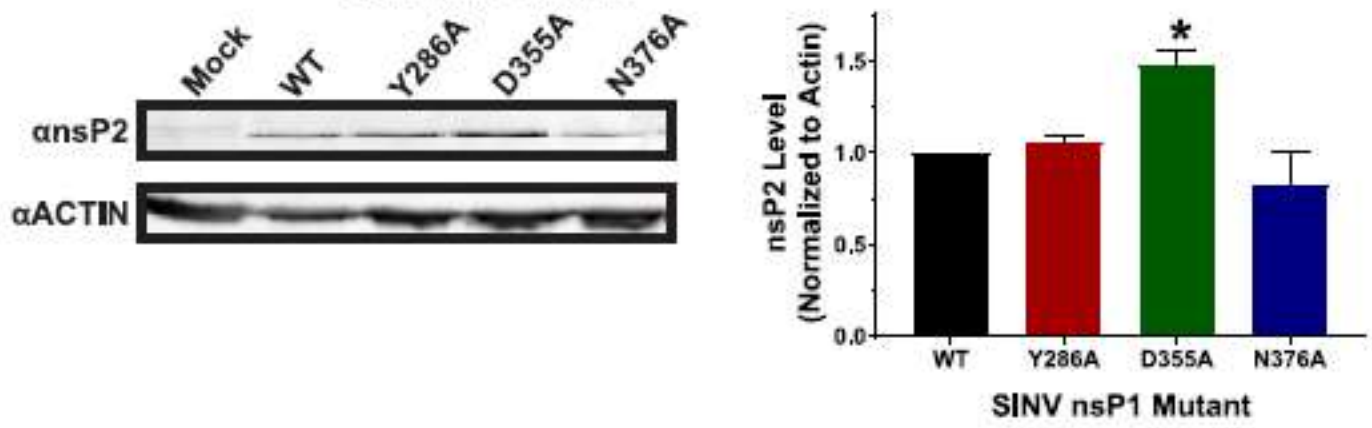

Figure 2.4. nsP2 protein levels are impacted by mutation of the nsP1

protein. (A) BKH-21 cells were infected with wild type SINV or an individual capping mutant at an $\mathrm{MOI}$ of $5 \mathrm{PFU} /$ cell and assessed by western blotting to determine the abundance of nsP2 at 8hpi. Actin is shown as a loading control. B) Densitometric quantification of the nsP2 protein normalized to Actin levels at 
8hpi. Panels $C$ and D) Western blots and densitometry analyses identical to those described for panels $A$ and $B$, with the exception that the timing of the assay coincided to $12 \mathrm{hpi}$. Panels E and F) Western blots and densitometry analyses identical to those described for panels $A$ and $B$, with the exception that the timing of the assay coincided to $16 \mathrm{hpi}$. The Western blot images shown are representative of at least three independent biological replicates. All the quantitative data shown represent the means of three independent biological replicates, with the error bars representing standard deviations of the mean. Statistical significances, as indicated in the figure, were first determined using ANOVA analyses followed by post-hoc statistical analyses by Student's $t$ test. 

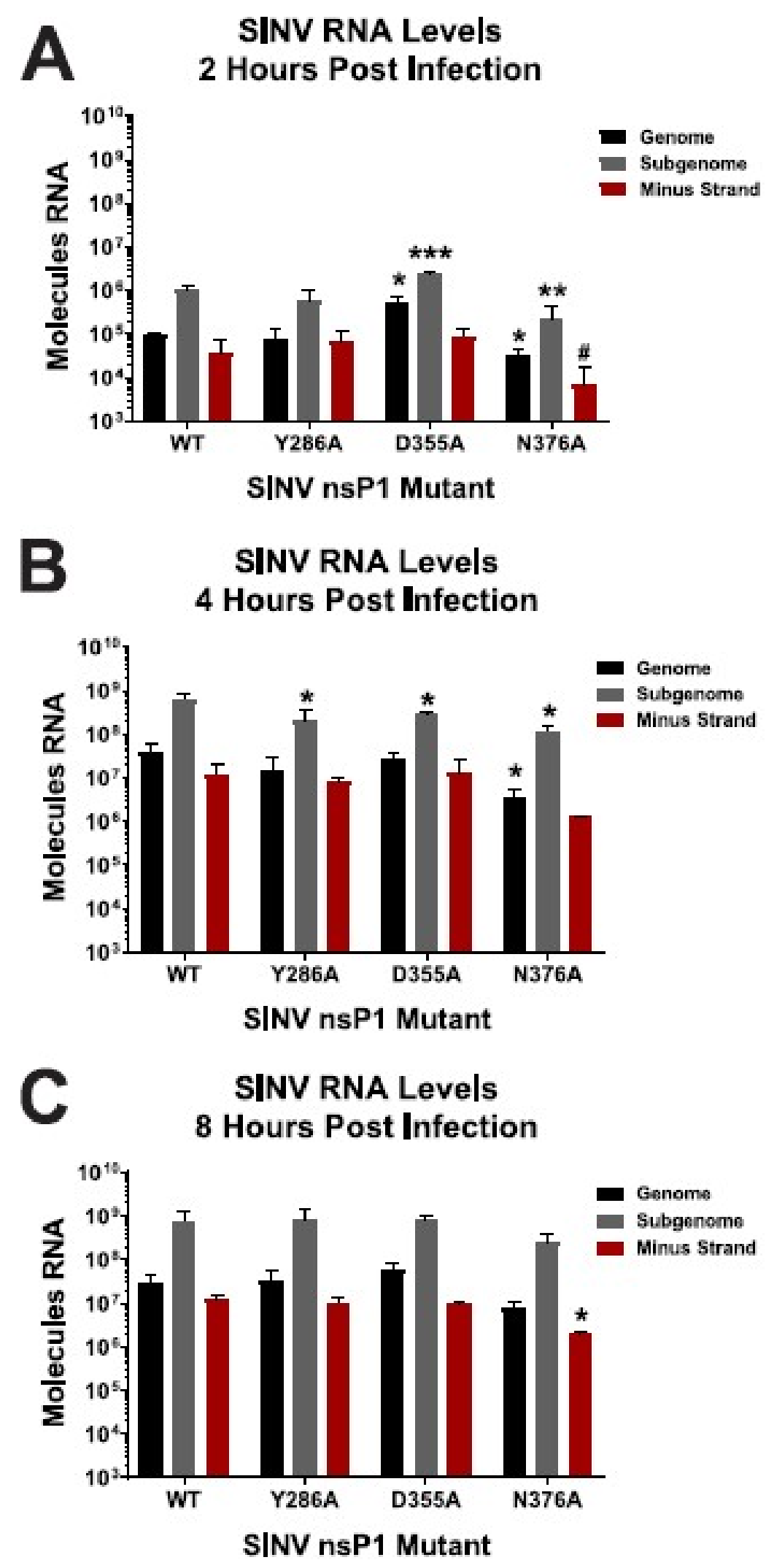
Figure 2.5. Altering vRNA capping efficiency impacts early RNA synthesis.

(A) BHK-21 cells were infected with either wild type parental SINV or an individual capping mutant virus at an $\mathrm{MOI}$ of $5 \mathrm{PFU} /$ cell. At 2 hours post infection, the total cellular RNA was extracted and assessed for the absolute quantities of the genomic, subgenomic, and minus-strand vRNAs by qRT-PCR. (B and C) Identical to panel A with the exceptions that the time points are 4 and 8 hours post infection, respectively. All the quantitative data shown represent the means of three independent biological replicates with the error bars representing standard deviations of the mean. Statistical significances, as indicated in the figure, were first determined using ANOVA analyses followed by post-hoc statistical analyses by Student's $t$ test. P-Values as determined by Student's $t$ test are represented by ${ }^{*}, p<0.05 .{ }^{* *}, p<0.01 .{ }^{* * *}, p<0.001 . \#$, one of the biological replicates was below limit of detection precluding meaningful statistical analysis. 

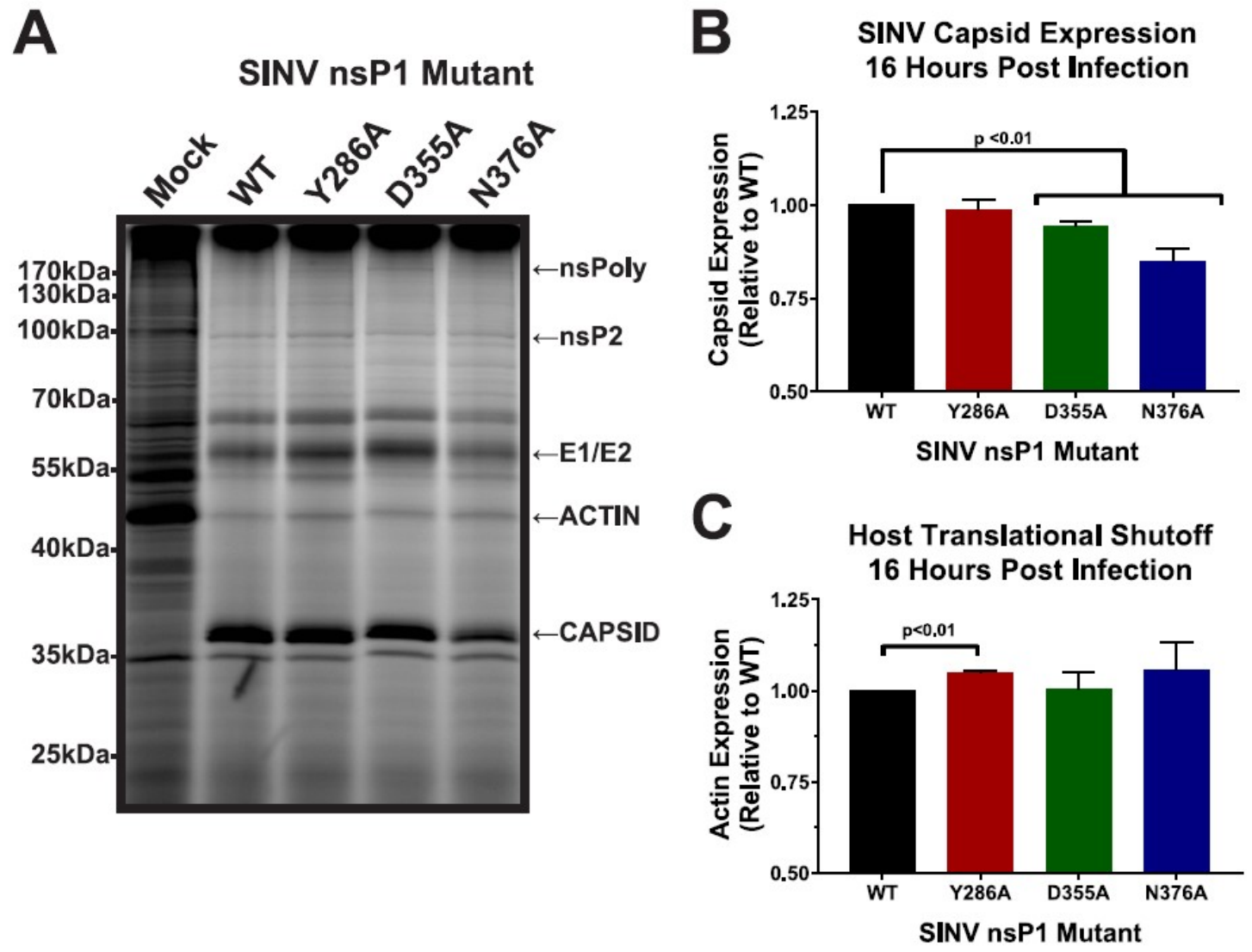

Figure 2.6. Subgenomic gene expression is unaffected by altering SINV

vRNA capping. (A) BHK-21 cells were either mock treated or infected with wild type SINV or an individual capping mutant at an MOI of $10 \mathrm{PFU} /$ cell. At $14 \mathrm{hpi}$, the cells were pulsed with L-AHA for a period of 2 hours. Afterwards, the cells were harvested and equal cell volumes of cell lysate were analyzed by SDSPAGE and fluorescent imaging. The data shown are representative of three independent biological replicates. (B) Densitometric quantification of the SINV capsid protein, with intensity relative to wild-type SINV shown. (C) Densitometric quantification of the host actin protein with intensity relative to wild type SINV. All the quantitative data shown represents the means of three independent 
biological replicates with the error bars representing standard deviations of the mean. Statistical significance was determined by Student's $t$ test. 

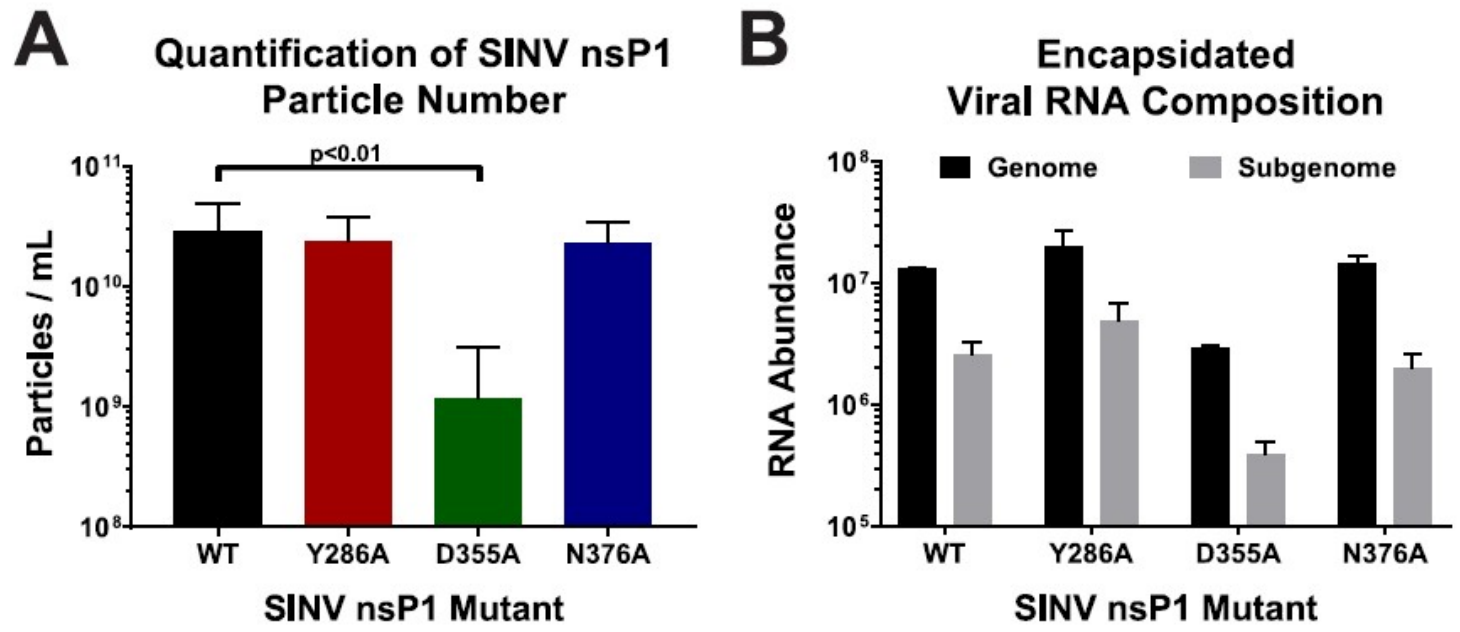

Figure 2.7. Analysis of SINV particle production. A) BHK-21 cells were infected with either wild-type SINV or an individual capping mutant at an $\mathrm{MOI}$ of 5 PFU/cell. At 24hpi the total number of viral particles produced was measured using qRT-PCR. Data shown represents the means of at least 6 independent biological samples. B) Quantitative determination of the composition of the encapsidated viral RNAs in mature extracellular viral particles. Samples of virus containing supernatants were assessed to determine the absolute quantities of the genomic and subgenomic RNAs via standard curve qRT-PCR. All the quantitative data shown represent the means of three independent biological replicates, with the error bars representing standard deviations of the mean. Statistical significances, as indicated in the figure, were first determined using ANOVA analyses followed by post-hoc statistical analyses by Student's $t$ test. 


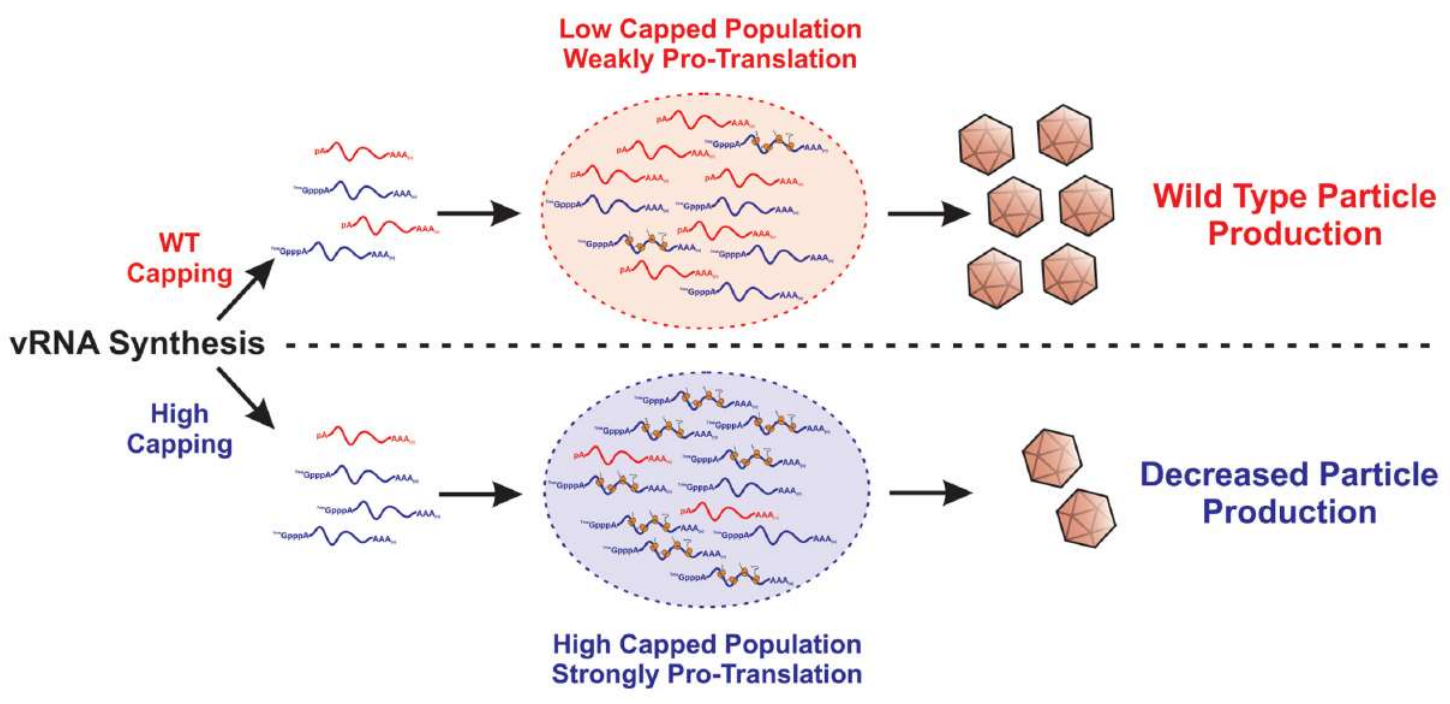

Figure 2.8. Proposed model of how increasing genomic vRNA capping negatively impacts viral infection. Wild-type SINV infection produces a low capped population of vRNAs that are weakly pro-translational and enables efficient particle production (top). Conversely, the increased capping nsP1 mutant D355A produces a population of vRNAs that are largely capped and therefore strongly pro-translational, which subsequently leads to impaired particle production compared to wild-type infection. 
CHAPTER 3

\section{PRODUCTION OF NONCAPPED GENOMIC RNAS IS CRITICAL TO SINDBIS VIRUS DISEASE AND PATHOGENICITY²}

\section{Summary}

Alphaviruses are positive-sense RNA viruses that utilize a 5' cap structure to facilitate translation of viral proteins and to protect the viral RNA genome. Nonetheless, significant quantities of viral genomic RNAs that lack a canonical 5' cap structure are produced during alphaviral replication and packaged into viral particles. However, the role/impact of the noncapped genomic RNA (ncgRNA) during alphaviral infection in vivo has yet to be characterized. To determine the importance of the ncgRNA in vivo, the previously described D355A and N376A nsP1 mutations, which increase or decrease nsP1 capping activity respectively, were incorporated into the neurovirulent AR86 strain of Sindbis virus to enable characterization of the impact of altered capping efficiency in a murine model of infection. Mice infected with the N376A nsP1 mutant exhibited slightly decreased rates of mortality and delayed weight loss and neurological symptoms, although levels of inflammation in the brain were similar to wild type infection. Although the

\footnotetext{
${ }^{2}$ This chapter is adapted from work published in mBio, 2020, Volume 11, Issue 6 with the same title under a Creative Commons Attribution (CC BY) license 163. LaPointe, A.T., et al., Production of Noncapped Genomic RNAs Is Critical to Sindbis Virus Disease and Pathogenicity. mBio, 2020. 11(6).
} 
D355A mutation resulted in decreased antiviral gene expression and increased resistance to IFN in vitro, mice infected with the D355A mutant showed significantly reduced mortality and morbidity compared to mice infected with wild type virus. Interestingly, expression of proinflammatory cytokines was found to be significantly decreased in mice infected with the D355A mutant, suggesting that capping efficiency and the production of ncgRNA are vital to eliciting pathogenic levels of inflammation. Collectively, these data indicate that the ncgRNA have important roles during alphaviral infection and suggest a novel mechanism by which noncapped viral RNA aid in viral pathogenesis.

\section{Introduction}

Alphaviruses are positive-sense, single-stranded RNA arboviruses that are capable of causing severe disease. The natural enzootic transmission cycle of these viruses is between a mosquito vector and a mammalian host, typically rodents or birds, although epizootic spillover events can occur that result in infection of humans and equids. Alphaviruses are broadly categorized as either arthritogenic or encephalitic based on disease symptomology. The arthritogenic alphaviruses, such as Chikungunya virus (CHIKV) and Ross River Virus (RRV) are capable of causing disease ranging from mild febrile illness to severe polyarthralgia, which can persist anywhere from weeks to years following infection [4, 11, 164]. In contrast, the encephalitic alphaviruses, such as Venezuelan Equine Encephalitis virus (VEEV) and some strains of Sindbis virus (SINV), like the AR86 strain used in this study, can cause mild to severe 
neurological symptoms, including encephalitis that can potentially lead to the death of the host $[11,12,165]$. While alphaviruses pose a large threat to public health, there are currently no safe and effective vaccines or antiviral therapies to prevent or treat alphaviral disease.

Alphaviruses produce three RNA species during infection: the genomic strand, which encodes the nonstructural proteins; the minus-strand RNA template; and the subgenomic RNA, which encodes the structural proteins. Both the genomic and subgenomic RNAs have a type 0 cap structure added to their 5' ends to facilitate translation and protect the 5 ' end of the transcripts [129-131]. The addition of the cap structure to the 5' end of viral RNAs is primarily carried out by nonstructural proteins 1 and 2 (nsP1, nsP2). NsP2 removes the $5^{\prime} \gamma^{-}$ phosphate from the nascent vRNA molecule while, in a separate reaction, the methyltransferase domain of nsP1 catalyzes the addition of a methyl group from S-adenosylmethionine to a GTP molecule, forming a covalent m7GMP-nsP1 intermediate $[40,132]$. The m7GMP moiety is then transferred to the 5'end of the vRNA molecule by the guanylyltransferase activities of nsP1, resulting in the 7meGppA type 0 cap structure [133].

In response to the lack of preventatives or treatments, targeting the alphaviral replication machinery has been a popular approach for developing potential antiviral therapies. Capping of the genomic and subgenomic vRNA is vital for successful viral replication, as mutations which completely inhibit capping of the viral RNA render the virus noninfectious. Thus, because nsP1 is responsible for the alphaviral capping process, it has been a popular target for 
antiviral research. In particular, a number of compounds have been developed which inhibit nsP1 capping activity and reduce viral replication in vitro, but, to date, none have been tested for efficacy against alphaviral infection in vivo [166169]. In addition to the development of drugs against nsP1 activity, multiple residues in nsP1 have also been identified as determinants for alphaviral virulence, however the impact of these residues on alphaviral capping efficiency has never been delineated. The SINV nsP1/nsP2 cleavage mutant T538I has been shown to determine pathogenicity in mouse models of infection by altering nonstructural polyprotein processing and the virus' sensitivity to interferon [101, 170]. More recently, a group of six mutations in the nsP1 of RRV have also been shown to attenuate alphaviral disease in mice, although the mechanism of attenuation and the impacts of these mutations on alphaviral replication have yet to be fully characterized $[97,98]$. These studies illustrate the significance of nsP1 to alphaviral infection and pathogenicity, but have yet to determine the importance of alphaviral capping efficiency and the production of the ncgRNAs to in vivo infection.

While capping of the viral RNA is critical to viral protein expression and viral replication, we have previously shown that the genomic vRNA are not universally capped, and that a significant proportion of the alphaviral genomic RNA produced and packaged during infection lack the 5' cap structure [99]. In addition, our recently published study showed that the proportion of noncapped genomic RNA (ncgRNA) produced during SINV infection could be altered using point mutations in nsP1 to modulate capping activity [171]. Specifically, 
incorporating a D355A mutation in the nsP1 of SINV resulted in increased capping efficiency, and therefore decreased ncgRNA production, relative to wild type SINV. Alternatively, a N376A mutation in nsP1 resulted in decreased capping efficiency and increased ncgRNA production. By utilizing these mutations to alter ncgRNA production, we were able to show that increasing the capping efficiency of nsP1 was detrimental to SINV infection in tissue culture models of infection, while decreasing nsP1 capping efficiency did not significantly affect viral titer or overall replication.

However, the presence or lack of a phenotype in vitro is not always indicative of what will occur during infection in vivo. As such, the goal of this study was to determine the effect of altered ncgRNA production on alphaviral pathogenesis by using the previously described nsP1 capping mutants in a mouse model of infection. The data presented here show that modulating ncgRNA production through the use of the D355A and N376A point mutations to alter nsP1 capping efficiency in nsP1, has a profound impact on alphaviral pathogenesis. In specific, decreasing capping efficiency resulted in increased sensitivity to type-I IFN and a slight decrease in mortality. Surprisingly, increasing capping efficiency resulted in almost complete abrogation of morbidity and mortality, despite showing increased resistance to type-I IFN, due to reduced immune infiltration and production of inflammatory cytokines in the brain. Collectively, our findings indicate that the ncgRNA are important in determining the host immune response to viral infection and play a critical novel role in alphaviral pathogenesis. 


\section{Materials and Methods}

Tissue culture cells. Baby Hamster Kidney 21 (BHK-21) fibroblast cells (a gift from Dr. R. W. Hardy, Indiana University - Bloomington) and interferon competent L929 mouse fibroblast cells (a gift from Dr. P. Danthi, Indiana University - Bloomington) were maintained in minimal essential media (MEM; Cellgro) containing fetal bovine serum (FBS; Atlanta Biologicals), 1\% PenicillinStreptomycin (Cellgro), 1\% nonessential amino acids (Cellgro), and 1\% Lglutamine (Cellgro). SK-N-BE(2) human nerve cells (a gift from Dr. L. Beverly, University of Louisville) were maintained in Dulbecco's Modified Eagle Medium (DMEM)/F12 media containing 10\% FBS, 1\% Penicillin-Streptomycin, and 1\% Lglutamine. All cell lines were cultured at $37^{\circ} \mathrm{C}$ and $5 \% \mathrm{CO}_{2}$ in a humidified incubator. Regular passaging using standard subculturing techniques was used to maintain low passage stocks.

Generation of AR86 SINV capping mutants. The AR86 SINV nsP1 mutants used in this study were generated by Gibson Assembly via the use of a Gibson Assembly HiFi 1-step kit (SGI), using a restriction-digested AR86 cDNA plasmid and a synthetic DNA fragment, according to manufacturer's instructions [165]. Mutants were verified by whole-genome sequencing; full-genome sequences are available upon request. 
Production of wild-type and mutant SINV Stocks. Wild-type, D355A, and N376A SINV AR86 were prepared by electroporation, as previously described [137]. Approximately $2.8 \times 10^{6} \mathrm{BHK}-21$ cells were electroporated with $10 \mu \mathrm{g}$ of in vitro-transcribed RNA. This was done using a single pulse at $1.5 \mathrm{kV}, 12 \mathrm{~mA}$, and $200 \Omega$ from a Gene Pulse Xcell system (BioRad) as previously described [171]. Afterwards, cells were incubated under normal conditions until cytopathic effect was apparent, at which point the supernatant was collected, clarified via centrifugation at $10,000 \mathrm{xg}$ for $10 \mathrm{~min}$ at $4^{\circ} \mathrm{C}$, and aliquoted into small volume stocks which were stored at $-80^{\circ} \mathrm{C}$ for later use.

Capping Assay. To define the impact of the nsP1 mutations on the capping activity of the alphaviral replicase complex, a linker-ligation mediated approach was used. Briefly, BHK-21 cells were infected with the aforementioned SINV AR86 nsP1 mutants at an Multiplicity of Infection (MOI) of 5 Infectious Units (IU) per cell, and at 16 hours post infection (hpi) total RNA was extracted from the cells via TRIzol. As our previous studies have indicated that the 5' end of the noncapped viral transcripts are poly-phosphorylated, the total RNA samples must be initially dephosphorylated prior to completing the linker-ligation method (which requires a 5' monophosphate) [99]. To this end, 1ug of RNA was dephosphorylated via treatment with Antarctic Phosphatase (NEB, M0289S) as per the manufacturer's instructions. After a 30 minute incubation period at $37^{\circ} \mathrm{C}$, the Antarctic Phosphatase was heat inactivated by incubating the reaction mixture for 2 minutes at $80^{\circ} \mathrm{C}$ prior to rapid cooling on ice. The dephosphorylated 
RNAs were then equally divided into two reactions to further prepare the noncapped and capped transcripts for linker-ligation and qRT-PCR analysis. To enable the detection of the noncapped viral RNAs, the dephosphorylated RNAs were treated with T4 Polynucleotide Kinase (PNK; NEB, M0201L) as per the manufacturer's instructions. In parallel, the capped RNAs were further prepared for linker-ligation via incubation in the presence of RNA 5' Pyrophosphohydrolase (RppH; NEB, M0356S), as per the manufacturer's instructions. Both the T4 PNK and $\mathrm{RppH}$ reactions were incubated for a period of 30 minutes at $37^{\circ} \mathrm{C}$ prior to phenol chloroform extraction and ethanol precipitation. The RNA pellets were resuspended in $20 \mathrm{ul}$ of nuclease-free water and used as the input materials for the linker-ligation reaction described below to enable the detection of the noncapped and capped vRNAs via qRT-PCR.

The prepared RNAs were then ligated to an RNA linker that was blocked on the 5' end with a 9 carbon spacer (RNA Linker- 5'-5Sp9GUUCAGAGUUCUACAGUCCGACCCAUC-3') via T4 RNA Ligase 1. Briefly, each 30ul reaction mixture consisted of 0.5 ugs of prepared RNA (as described above), 1X T4 RNA Ligase Buffer, 1mM ATP (final concentration), 1.66uM RNA Linker Oligo (final concentration), 10 Units (U) of T4 RNA Ligase 1 (NEB, M0204S), and 40U of RNAse Inhibitor (NEB, M0314S). The linker-ligation reactions were incubated at $25^{\circ} \mathrm{C}$ for a period of 2 hours prior to phenol chloroform extraction and ethanol precipitation. The linker-ligated RNAs were resuspended in 20ul of nuclease-free water, and $5 \mathrm{ul}$ of the ligated RNAs were used as the input for Reverse Transcription (RT) reactions using OneScript Plus 
RT (Abmgood, G237) as per the manufacturer's instructions. To enable the specific amplification of the viral genomic RNA, the RT reactions were primed with SINV.nSP1.R oligo (5'-AACATGAACTGGGTGGTGTCGAAG-3'). The composition of the SINV genomic RNA 5' ends were then quantitatively assessed via qRT-PCR as previously described using primer sets specific for the RNA linker as well as for nsP1 [99]. The amount of linker-containing genomic RNAs relative to the total genomic RNA level was then calculated using the $\Delta \Delta C_{T}$ method in order to determine the ratio of capped or noncapped RNA present, depending on the sample treatment described above. The primers used for this experiment are listed in Supplemental Table 1.

Analysis of viral growth kinetics. To determine if the mutation of the SINV nsP1 protein negatively impacted AR86 SINV infection, one-step viral growth kinetics for each capping mutant were assayed in tissue culture models of infection. BHK-21 cells were seeded in a 12-well plate and incubated under normal conditions until cell monolayers were $80-90 \%$ confluent. The cells were then infected with either wild-type virus or the individual capping mutant virus at an $\mathrm{MOI}$ of $5 \mathrm{IU} / \mathrm{cell}$ and the virus was allowed to adsorb for $1 \mathrm{~h}$. The inoculum was then removed, the cells were washed with $1 \mathrm{x}$ phosphate buffered saline (PBS) to remove any unbound viral particles, and whole medium supplemented with $25 \mathrm{mM}$ HEPES was added. The cells were incubated at $37^{\circ} \mathrm{C}$ and tissue culture supernatants were harvested (and the media replaced) at the indicated times post infection. Viral titer was then determined via plaque assay. 
Quantification of infectious virus by plaque assay. In order to determine infectious viral titer of all viral samples produced during this study, standard virological plaque assays were used. To summarize, BHK-21 cells were seeded in 24-well plates under normal incubation conditions until the cell monolayers were $80-90 \%$ confluent. At that point, the cells were inoculated with $10-$ fold serial dilutions of virus containing samples followed by a 1-hour adsorption period. Afterwards, cells were overlaid with a solution of $0.5 \%$ Avicel (FMC Corporation) in $1 \mathrm{x}$ media for $48 \mathrm{~h}$ [138]. The monolayers were then fixed with formaldehyde solution ( $3.8 \%$ formaldehyde-1x PBS) for at least $1 \mathrm{~h}$. The overlay was then removed, and the plaques were visualized via crystal violet staining.

Western Blot. To determine whether or not altering the capping efficiency impacted the expression of the SINV nonstructural genes, the expression of nsP2 was assessed via Western blotting. Briefly, whole-cell lysates were generated from BHK-21 cells that were infected with either wild type SINV AR86, one of the aforedescribed nsP1 mutants, or Mock infected. At 8 hours post infection, the cells were lysed via the addition of RIPA buffer (50mM Tris-HCL $(\mathrm{pH} 7.5)-50 \mathrm{mM} \mathrm{NaCl}-1 \%(\mathrm{v} / \mathrm{v})$ Nonidet P40 (NP-40) - 0.5\% (w/v) SDS $0.05 \%(\mathrm{w} / \mathrm{v})$ sodium deoxycholate $-1 \mathrm{mM}$ EDTA) followed by vigorous vortexing prior to storage at $-80^{\circ} \mathrm{C}$ until further use. Equal amounts of whole-cell lysates were resolved using SDS-PAGE and transferred to nitrocellulose membranes for downstream immunodetection. The resulting blots were probed for anti-SINV 
nsP2 polyclonal sera (a gift from R.W. Hardy at Indiana University-Bloomington) and anti-Actin (ThermoFisher, clone mAGGEa), and probed with the appropriate HRP-labeled secondary antibodies using the iBind Flex Western System with HRP detection / blotting reagents (ThermoFisher). Detection of the SINV nsP2 and host Actin proteins was accomplished via chemiluminescence with SuperSignal West Pico PLUS Chemiluminescent Substrate (ThermoFisher, 34579) detected by an Azure C200 Imaging Station (Azure Biosystems, C200).

RNA Kinetics. BHK-21 cells were infected with either wild type SINV AR86, or one of the aforementioned SINV nsP1 mutants at an $\mathrm{MOI}$ of $5 \mathrm{IU} /$ cell. At the indicated times post-infection, the total RNA was isolated from the infected cells via TRIzol reagent, as according to the manufacturer's instructions. Paired RT reactions were assembled using 1 ug of total cellular RNA and primer sets designed to prime the synthesis of cDNA from the viral RNA species in a transcript specific manner. Briefly, the positive-sense RNAs were primed for cDNA synthesis using SINV.nsP1.R (5'-AACATGAACTGGGTGGTGTCGAAG-3') and SINV.E1.R (5'-ATTGACCTTCGCGGTCGGATACAT-3'), and the negative sense RNAs were primed for cDNA synthesis using SINV.nsP1.F (5'AAGGATCTCCGGACCGTA-3'). All RT reactions also included an oligo priming for the mammalian 18S rRNA, Mam.18S.R (5'-AGTCGGCATCGTTTATGGTC3'). qRT-PCR detection of the viral RNA species was accomplished using a standard curve analysis and subtractive method as previously described [171]. To determine the amount of subgenomic vRNA present, the absolute quantities 
of RNA detected with the nsP1 primer set were subtracted from that detected with the E1 primer set, as the E1 primer set will detect both the genomic and subgenomic RNAs, but the nsP1 primer set will only detect the genomic RNA. Primer pairs are listed in Supplemental Table 1.

Type-I IFN sensitivity assay. L929 cells were seeded in a 48 well plate and, upon reaching $80-90 \%$ confluency, were inoculated with either wild-type parental virus or one of the individual capping mutants at an MOI of $10 \mathrm{IU} /$ cell. After a $1 \mathrm{~h}$ adsorption period, the inoculum was removed, the cells were washed twice with 1x PBS, and whole media was added. At the indicated times post infection, 20 international units (IU) of murine type-I IFN (R\&D Systems) was added to the media. Supernatants were collected at $24 \mathrm{hpi}$ and viral titer was determined by plaque assay. To calculate the relative sensitivity, the difference in viral titer between the type-I IFN treated and untreated infections was calculated at each time point for each virus. These differences were then calculated relative to the differences seen during wild-type SINV infection in order to determine relative sensitivity to type-I IFN.

Detection of ISG and IFN $\beta$ transcripts. To determine abundance of IFN $\beta$ and the listed ISG transcripts, L929 cells were seeded in a 24-well plate and, upon reaching $80-90 \%$ confluency, were inoculated with wild-type SINV or one of the capping mutants at an MOI of $10 \mathrm{IU} /$ cell. Additionally, L929 cells were mock infected with PBS to determine baseline IFN $\beta$ and ISG expression. After a $1 \mathrm{~h}$ 
adsorption period, the inoculum was removed, the cells were washed once with 1x PBS, and whole media was added. At the specified timepoints, media was removed, the cells were washed once with 1x PBS, and cell lysates were harvested and RNA was extracted using acidic guanidinium thiocyanate-phenolchloroform extraction [172]. The RNA was then DNAse treated and precipitated via phenol-chloroform extraction. Following precipitation, $1 \mu \mathrm{g}$ of RNA was reverse transcribed using random hexamer primer and QRT-PCR was carried out as described above with primer sets obtained from PrimerBank. The sequences of these primers may be found in the Supplemental Materials associated with this manuscript. Data was first normalized to GAPDH to account for any differences in total RNA level between samples. Data from the wild-type SINV, D355A, and N376A infections were then also normalized to nsP1 to account for differences in viral RNA levels that were present. Fold change in IFN $\beta$ and ISG expression was then calculated relative to what was observed during mock infection.

Mouse Experiments. Four-week old C57BL/6 mice were obtained from Jackson Laboratory and were inoculated in the left, rear footpad with 1000 PFU of virus in diluent (1x PBS) in a volume of 10 $\mu \mathrm{L}$. Mock-infected animals were injected with diluent alone. Mice were monitored for neurological signs of disease and weighed twice daily. A total of 130 mice were used in this study. For mortality studies, 2 biological replicates were completed with 4 animals were used per group, with a total of 4 groups. For titer studies, 6 animals were used per group, with a total of 3 groups, and a total of 5 timepoints. Eight additional mock infected 
mice were harvested and paired with the above timepoints. Neurological scoring was as follows: $0=$ No signs of overt disease, and normal behavioral activity; $1=$ Abnormal trunk curl, grip, or tail weakness (1 of 3); 2 = Abnormal trunk curl, grip, or tail weakness (2 of 3); 3 = Absent trunk curl, lack of gripping, tail paralysis; $4=$ Pronounced dragging of one or more limbs; $5=$ hind or fore limb paralysis. On the termination day for each experiment or when mice met endpoint criteria (neurological scores of 5 or 4 if the animal was unable to obtain food or water), or weight loss greater than $20 \%$ of initial body weight, the mice were sedated with isoflurane and euthanized by thoracotomy. Blood was then collected and serum obtained by collecting blood in serum separator tubes. Following exsanguination, tissues were collected by dissection. Tissues were then placed in 1x PBS and homogenized using Kimble BioMasher II closed system micro tissue homogenizers. Ankle tissue was processed by bead beating using a Bead Ruptor 4 (Omni International). The infectious virus present in the tissue was quantified by plaque assay.

For histology, uninfected and SINV infected mice brains were removed at day 7 post infection and were divided in half sagitally. One half was used to assess viral titer (described above), while the remaining half was fixed in $4 \%$ formaldehyde and sectioned in paraffin. Tissue sections were then stained with haematoxylin and eosin (H\&E). Pathological changes were scored by a board certified veterinary pathologist (through the Comparative Pathology Core Services facility, lowa State University) in the indicated categories and regions of 
the brain were scored as follows: $0=$ normal, $1=$ minimal, $2=$ mild, $3=$ moderate, 4=severe.

\section{Detection of viral genome and cytokine transcripts in mouse tissues. To} measure the level of viral genome and cytokine transcripts in tissues of infected mice, RNA was extracted from tissue homogenate using acidic guanidinium thiocyanate-phenol-chloroform extraction. The RNA was then DNAse treated and precipitated via phenol-chloroform extraction. Following precipitation, $1 \mu \mathrm{g}$ of RNA was reverse transcribed using Protoscript II reverse transcriptase (NEB) and random hexamer primer. The RNA genome was detected using BrightGreen Express qPCR master mix (Abmgood) and the following primer set specific for nsP1= F: 5-AAGGATCTCCGGACCGTA-3, and R: 5-

\section{AACATGAACTGGGTGGTGTCGAAG-3. A standard curve of known}

concentrations was used to determine the absolute quantities of viral genomic RNAs. Cytokine transcripts were detected using TaqMan Fast Advanced Master Mix and the Applied Biosystems TaqMan Array Mouse Immune Response plates (Catalog number:4414079) according to manufacturer's instructions.

Neuron Viability. Neuron viability was determined using a previously described method of ethidium bromide and acridine orange staining [173-175]. SK-N-BE(2) cells were seeded in a 96-well plate and, upon reaching $80-90 \%$ confluency, were inoculated with either wild-type SINV or one of the capping mutants at an $\mathrm{MOI}$ of $30 \mathrm{IU} / c e l l$. After a $1 \mathrm{~h}$ adsorption period, the inoculum was removed, the 
cells were washed once with $1 \mathrm{x}$ PBS, and DMEM/F12 media was added. At 24hpi, cell viability was assessed using ethidium bromide/ acridine orange staining described in Ribble et al. Briefly, the 96-well plate was centrifuged at 1,000 RPM for 5 min using an Allegra 25R model centrifuge (Beckman Coulter) with inserts for 96-well plates. Following centrifugation, $8 \mu \mathrm{L}$ of EB/AO dye solution $(100 \mu \mathrm{g} / \mathrm{mL}$ ethidium bromide and $100 \mu \mathrm{g} / \mathrm{mL}$ acridine orange in $1 \times$ PBS) was added to each well. Cells were viewed using an epifluorescence microscope. Tests were done in triplicate and a minimum of 100 total cells per well were counted using ImageJ.

Animal Ethics and Research. This study was carried out in strict accordance with the recommendations described in the Guide for the Care and Use of Laboratory Animals of the National Institutes of Health. The protocol was approved by the Institutional Animal Care and Use Committee of the University of Louisville (Approval \#17-140). All manipulations which could result in acute pain or distress were performed under isoflurane anesthesia.

Statistical Analysis. Unless otherwise stated, the quantitative data presented in this study represent the means of data from a minimum of three independent biological replicates. The in vivo studies described in this study were performed in duplicate using two independent preps of viral stocks. An area-under-the-curve approach was used to statistically assess the growth curve data presented in Figure 3.1D to determine the differences in viral growth kinetics through the 
course of the assay. Comparative samples were statistically analyzed as previously described [137], using variable bootstrapping where appropriate. The survival data presented in Figure 3.6 were statistically analyzed using the log rank test. Student's $t$ test was used to determine the $P$ values associated with individual quantitative data sets. Significance for data presented in Figure 3.10 was determined using the Benjamini and Hochberg correction, and the corrected p-Values are shown in Fig. 3.10 panel B.

\section{$\underline{\text { Results }}$}

\section{Altering Capping Efficiency Is Detrimental To Viral Growth Kinetics In Neurovirulent SINV In Vitro.}

Given our previously reported findings describing the molecular phenotypes of the nsP1 mutants in tissue culture models of infection, we were interested in characterizing how altering ncgRNA production impacted SINV infection in vivo. However, our previous characterizations of the capping mutants were done using a Toto1101-derived strain of SINV, which is tissue culture adapted and does not cause disease in adult wild type mice. Rather than rely on very young mice or mouse models with deficiencies in IFN competency to assess pathogenesis, we elected to change the SINV strain used to allow assessments in adult wild type mice. Thus, we incorporated the D355A and N376A nsP1 mutations into the AR86 background of SINV, a neurovirulent strain capable of causing lethal disease in an adult mouse model. 
To confirm that these point mutations had similar phenotypes in the AR86 background as compared to the previously used Toto1101-derived background, the proportion of capped and noncapped genomic RNAs were quantified in BHK21 cells at $16 \mathrm{hpi}$ (Figure $3.1 \mathrm{~A}-\mathrm{C}$ ). For wild type AR86 SINV, approximately half of the genomic RNA was found to be capped. This is consistent with what was previously found for the Toto1101-derived SINV, which also exhibited a capped:noncapped ratio of $1: 1$ at the equivalent time during infection [99]. Specifically, the D355A nsP1 mutation resulted in production of an RNA population consisting of 4-fold more capped RNA than noncapped RNA, whereas the N376A nsP1 mutation produced a population with 4-fold more noncapped RNA than capped RNA (Figure 3.1A). When compared to wild type AR86, the D355A mutant produced significantly more capped vRNA and significantly less ncgRNA (Figure 3.1B and C). Conversely, the N376A mutant produced significantly less capped vRNA and significantly more ncgRNA compared to wild type SINV. Although the absolute magnitude by which capping efficiency was affected by the nsP1 mutations appears to be different in the AR86 strain compared to that observed for the previously used Toto1101-derived strain, the trend of the D355A mutation increasing capping efficiency and the N376A mutation decreasing capping efficiency remained the same [171].

In addition to confirming the impact of the nsP1 mutations on vRNA capping in the AR86 background, viral growth kinetics were also assessed in BHK-21 cells (Figure 3.1D). Similar to what we have reported previously, increasing the capping efficiency of nsP1 with the D355A mutation resulted in an approximately $1.5 \mathrm{log}$ 
decrease in viral titer over the course of infection. Likewise, decreasing capping efficiency with the N376A mutation also resulted in a significant decrease in viral growth kinetics. While the phenotype associated with the N376A mutant was more dramatic in the AR86 strain than what was reported in our previous study, this might be explained by the fact that the AR86 strain of SINV is not adapted for replication in tissue culture like the previously used Toto1101 strain. Therefore, the impact of the N376A mutation on replication is likely exacerbated in tissue culture systems for the AR86 strain, leading to the significantly reduced viral growth kinetics seen in Fig. 3.1D.

To further confirm that the nsP1 mutants did not introduce gross lifecycle defects in the AR86 background, viral translation was measured. To assess how the nsP1 capping mutations affected viral translation, BHK-21 cells were infected with either wild type SINV or one of the nsP1 capping mutants, and the relative expression of the SINV nsP2 protein was detected via Western blotting (Figure 3.2A). Densitometry analysis of the fully cleaved form of nsP2 revealed no quantitative differences across the SINV AR86 nsP1 mutant strains (Figure 3.2B). Despite no apparent differences in fully processed nsP2 levels, increased levels of nonstructural polyprotein were detected in the SINV nsP1 D355A mutant via comparative densitometry (Figure 3.2C). Furthermore, comparing the total signal detected by the anti-nsP2 polyclonal serum reveals slightly enhanced nonstructural gene expression in the D355A nsP1 mutant, consistent with our previous observations using the Toto1101-derived nsP1 mutants. These data are suggestive of increased or ongoing nonstructural protein synthesis in the D355A 
background. As supported by the equivalent levels of fully processed nsP2, the processing of the nonstructural polyprotein likely occurs at a rate that is unaffected by the level of translation of the polyprotein. Thus, increasing the capping efficiency of the nsP1 protein led to increased nonstructural gene expression. Unlike what was previously found, the N376A decreased capping mutant showed no difference in viral translation compared to wild type SINV.

On the basis of our previous examinations of the Toto1101-derived SINV nsP1 mutants, we postulate that differences in viral translation may be more pronounced during the earliest stages of infection. Previously we have utilized nanoluciferase reporter viruses to quantitatively assess viral gene expression in a highly sensitive manner. Unfortunately, the inclusion of a nanoluciferase reporter into the nsP3 protein of SINV AR86 has been found to be highly attenuating, precluding the detailed quantitative assessment of early SINV AR86 translation.

In addition to viral translation, viral RNA synthesis / accumulation was also assessed to determine if the nsP1 capping mutations affected RNA kinetics in the AR86 strain of SINV. Similar to what was shown in the Toto1101-derived background, there were no significant differences for any of the VRNA species produced by either nsP1 mutant at any of the measured timepoints compared to wild type SINV (Figure 3.3A-C) [171].

Taken together, we can conclude that the D355A and N376A nsP1 mutations affect capping efficiency and viral nonstructural protein expression in the AR86 strain of SINV in a manner that is similar to the previously used Toto1101derived background. We can also conclude that the decreases seen in viral titer 
between the nsP1 capping mutants and wild type SINV are not due to deficits in viral translation or RNA synthesis, but are rather due to changes in the proportion of ncgRNA produced by each mutant. Overall, the recapitulation of the original D355A and N376A capping phenotypes in the AR86 background provided a means by which the biological impact of the ncgRNAs on viral infection and pathogenesis could be assessed using an adult wild type mouse model. However, since our data suggest that the early events of the viral lifecycle may be altered by modulating capping efficiency, we decided to first characterize the engagement of the AR86-derived nsP1 mutants with the host innate immune response at a cellular level prior to utilizing a small animal model of SINV infection [171].

\section{Modulating ncgRNA Production Alters The Host Type-I Interferon Response To SINV Infection.}

The capacity of the host cell to detect viral infection and mount an antiviral response by upregulating interferon and interferon stimulated gene (ISG) expression is an important aspect of viral infection. As such, we were interested in determining whether changes in viral capping efficiency would impact the stimulation of the host type-I IFN response. To assess the extent to which the nsP1 mutants elicited an interferon response, interferon competent L929 cells were infected with either wild type SINV or one of the capping mutants and then the expression of IFN $\beta$ was measured at the transcriptional level using qRT-PCR (Figure 3.4A). For this experiment, in addition to quantitatively assessing IFN $\beta$ transcripts throughout the viral lifecycle, we also quantified the transcript 
abundance of select ISGs with well-established times of maximal expression post viral infection, so that we could more accurately determine the host antiviral response over the course of infection [176]. As such, CXCL10 and IFIH1 were used to determine the ISG expression at 6 and 8hpi, Viperin and MX2 at $16 \mathrm{hpi}$, and OAS2 and BST-2 at 24hpi (Figure 3.4B-E).

Infection with the D355A mutant resulted in significantly reduced IFN $\beta$ expression compared to wild type SINV at 8 and 16hpi, while N376A infection resulted in significantly greater IFN 3 expression at 6 and 24hpi (Fig. 3.4A). In regards to ISG transcript levels, infection with the N376A mutant resulted in significantly increased ISG expression compared to wild type SINV at 6hpi, but transcript abundance was roughly equivalent to wild type infection at 8hpi (Figure 3.4B-D). However, by $24 \mathrm{hpi}$, ISG expression levels were once again significantly increased (Figure 3.4E). In contrast, ISG expression levels in response to the D355A mutant was significantly decreased compared to wild type SINV until 24hpi, where OAS2 expression was still significantly decreased, but BST2 expression was similar to wild type.

Overall, infection with the increased capping mutant, D355A, led to a mostly decreased host antiviral response compared to what was seen during wild type infection, while the decreased capping mutant, N376A, elicited a response that was mostly increased compared to wild type SINV. Taken together, these results illustrate that modulating ncgRNA production has a significant impact on the induction of the host type-I IFN response. 


\section{Sensitivity To Type I IFN Correlates With Capping Efficiency In Tissue Culture.}

Given that altering capping efficiency affected viral gene expression, and altered the induction of the host type-I IFN response, we were interested to see if changes in ncgRNA production affected the virus's sensitivity to IFN treatment. In other words, we have shown that altering capping efficiency impacts how much IFN is produced, but does altering SINV capping efficiency affect the virus's capacity to resist IFN treatment when the cells are treated with equal amounts of type-I IFN? To determine whether changes in ncgRNA production affected the sensitivity of the nsP1 mutant viruses to exogenous type-I IFN, IFN-competent L929 cells were infected with either wild type or a SINV nsP1 mutant virus and recombinant type-I IFN was then added at $0,1,2,3$, or 4 hours post infection and viral titer was measured at 24hpi (Figures 3.5A-C) [175].

During wild type SINV infection, viral titer was reduced by $\sim 2$ logs when IFN was added at $0 \mathrm{hpi}$, with maximum titer increasing regularly as the time of IFN addition was delayed. IFN treatment at $4 \mathrm{hpi} \mathrm{did} \mathrm{not} \mathrm{impact} \mathrm{SINV} \mathrm{AR86} \mathrm{replication}$ as viral titers were equivalent to those of the no IFN control (Fig. 3.5A). The D355A mutant, which has increased capping efficiency and viral gene expression, was found to be significantly more resistant to type-I IFN treatment early during infection compared to wild type SINV, with viral titer only being reduced by $\sim 1$ log when IFN was added at $0 \mathrm{hpi}$ (Fig. 3.5B). As with wild type SINV, the maximal titer of the D355A mutant steadily increased as the addition of type-I IFN was delayed to later times post infection; however, the relative differences in titer between the 
IFN treated infections and non-treated control indicate that the D355A mutant was significantly more resistant to the impacts of type-I IFN overall. In contrast, the N376A mutant, which has decreased capping efficiency, was found to have wildtype equivalent sensitivity to IFN when added at 0 and $1 \mathrm{hpi}$, but the N376A mutant remained sensitive to the addition of type-I IFN at later times during infection (Figure 3.5C). Surprisingly, even when IFN was added as late as $4 \mathrm{hpi}$, the titers of the N376A mutant remained decreased compared to those of the IFN negative control, revealing that the N376A mutant remains sensitive to IFN for a longer period of time than wild type SINV.

To enable the comparative analysis of the impact of type-I IFN on the viral infections of the capping mutants compared to wild type infection, the differences in viral titers between the IFN treated and the control infections for each virus and time point were calculated. The differences in titer found for each nsP1 mutant were then made relative to the corresponding differences observed for the wild type infection (Fig. 3.5D). These data illustrate that during the early stages of viral infection the D355A nsP1 mutant is approximately 2-fold more resistant to type-I IFN than wild type SINV AR86; however, by 2 hpi the advantage has largely waned and the level of IFN resistance is similar to that observed during wild type infection. Similarly, comparing the resistance of the N376A mutant to wild type SINV AR86 further reveals that decreasing capping efficiency correlates with significantly increased sensitivity to type-I IFN up to at least 4 hours post infection.

It is interesting to note that, in the absence of IFN treatment, infections of the IFN-competent L929 cells with wild type SINV or the D355A mutant resulted in 
roughly equivalent viral titers at $24 \mathrm{hpi}$. This is different than what was observed previously in BHK-21 cells, where the D355A mutation resulted in significantly decreased viral titer (Fig. 3.1D). This difference in phenotypes between the two cell lines is likely due to the fact that the L929 cells are IFN competent and will produce IFN in response to viral infection while BHK-21 cells are incapable of doing so, resulting in differential viral replication rates due to the host response to viral infection in conjunction with the apparent differences in IFN sensitivity.

Overall, the sensitivity of the SINV nsP1 mutants to IFN reflects the differences seen in capping efficiency. Increased capping efficiency resulted in the D355A mutant being more resistant to type-I IFN early during infection when compared to wild type SINV. Likewise, decreased capping efficiency resulted in the N376A mutant being more sensitive to type-I IFN. This indicates that both the viral response to type-I IFN and the virus' ability to mitigate the effects of IFN expression on viral replication are altered depending on the level of ncgRNA produced during infection.

\section{Increasing Capping Efficiency Significantly Attenuates Neurotropic SINV In}

\section{A Mouse Model.}

As the capacity to avoid the elicitation of the host innate immune response, and the capacity to disregard the effects of the host type-I IFN response are vital to alphaviral replication and pathogenesis, the above data suggested that altering ncgRNA production could have profound effects on viral replication and pathogenesis in vivo. We hypothesized that, due to the nsP1 D355A mutant's 
increased resistance to type-I IFN and generally reduced activation of ISG expression, mice infected with the nsP1 D355A mutant would experience disease severity similar to wild-type SINV infection, perhaps with the mean survival time being potentially decreased due to increased IFN resistance. Conversely, we hypothesized that mice infected with the N376A mutant would experience more mild disease and decreased mortality because of the mutant's increased sensitivity to IFN and the greater expression of ISGs in response to infection in tissue culture models of infection. To test our hypothesis, we infected 4-week old male and female C57BL/6 mice with 1,000 PFU of SINV AR86 wild-type, nsP1 D355A, or nsP1 N376A via rear footpad subcutaneous inoculation. Mock infected mice were inoculated with PBS in the same manner. When infected with wild type SINV, adult C57BL/6 mice displayed significant weight loss as well as severe neurological symptoms, including rapid onset paralysis of the limbs, blindness, and seizures at approximately day 6 post infection (Figure 3.6A and B). Infection with wild type SINV also led to significant mortality, with infected mice having a mean survival time of $\sim 6$ days post infection (Figure $3.6 \mathrm{C}$ ). Likewise, mice experimentally infected with the decreased capping mutant N376A exhibited similar weight loss and neurological symptoms as wild type infected mice. However, the onset of disease in the N376A infected mice was delayed compared to wild-type SINV, with neurological symptoms starting at $5 \mathrm{dpi}$ and the mean survival time being $\sim 7 \mathrm{dpi}$, a full day later than what was seen with wild type SINV. In addition, a slightly greater proportion of mice survived when infected with the N376A mutant as opposed to wild type SINV. This increase in survival may be due to the delay in 
the N376A mutant to cause neurological symptoms, allowing the mice to be slightly older, and therefore better able to resist severe, lethal encephalitis $[80,177,178]$.

Surprisingly, the increased capping mutant virus D355A was significantly attenuated in mice. Compared to the previous two viruses, mice infected with the D355A mutant experienced minimal weight loss, milder neurological symptoms, and significantly reduced mortality, with all but one mouse surviving to the end of the study. Given that these mice did in fact show mild neurological and nonneurological symptoms and reduced weight gain compared to mock infected mice, we concluded that the nsP1 D355A increased capping mutant virus is indeed capable of causing pathogenesis, although the severity of disease is significantly reduced compared to wild type infection.

The trends seen in morbidity and mortality between the mice infected with wild type SINV versus the capping mutants were further reflected in H\&E stained sections of the brains of infected and uninfected mice (Figure 3.7). The brain sections of both wild type and N376A infected mice displayed numerous lesions consisting of lymphocytic meningitis; perivascular cuffing, which is indicative of immune cell infiltration; and neuronal apoptosis, which left open pockets in the tissue (Figure 3.7A). In addition, mice infected with either wild type SINV or the N376A mutant had significant pathology in terms of inflammation, neuronal degradation, and glial cell proliferation in multiple areas of the brain. Pathology was highest in the cerebrum and the midbrain / brainstem, but lesions were also found in the hippocampus and medulla oblongata of some mice (Figure 3.7B). Conversely, the brains of the D355A infected mice resembled mock infected mice, 
with no immune infiltration, cell death, or other signs of pathology in any area of the brain. Given that viral killing of neurons is the speculated cause of encephalitis and paralysis in SINV infection, the differences in tissue damage and neuron death seen between the D355A mutant and the other two viruses were not unexpected, as the D355A infected mice did not exhibit signs of encephalitis or limb paralysis (Figure 3.6B) [179, 180].

Collectively, these data suggest that modulating the production of ncgRNA has significant impacts on alphaviral pathogenesis. Overall, the nsP1 N376A point mutation, which increased ncgRNA production through decreased capping efficiency, resulted in delayed disease progression and mortality compared to wild type SINV in vivo. However, severity of neurological symptoms and pathology in the brain were unaffected. In contrast, the nsP1 D355A point mutation, which decreased ncgRNA production through increased capping efficiency, resulted in significantly decreased mortality, mild neurological symptoms, and little to no pathology in the brain. While the presence of mild symptoms and a lack of weight gain in the D355A infected mice do suggest that the virus was capable of trafficking to the brain and replicating, these data do not eliminate these as being possible reasons for the decreases in pathogenesis seen thus far.

\section{Attenuation Of Viral Pathogenesis Is Not Due To Deficits In Viral Dissemination Or Replication.}

Given that the D355A and N376A nsP1 mutants showed decreased viral titers in tissue culture model systems compared to wild type SINV (Figure 3.1D), 
we hypothesized that the reduction in mortality seen in Figure 3.6 was due to poor viral replication, dissemination, or a change in virus tropism for the brain. In order determine the impact of modulating capping efficiency on viral replication and to confirm that the nsP1 D355A mutant did in fact make it to the brains of infected mice, we measured viral titer at the site of inoculation and in the serum at $1 \mathrm{dpi}$ as well as in the brain at 7dpi. By comparing the viral titers of the nsP1 D355A mutant to wild type SINV in these tissues, we were able to determine if decreasing ncgRNA production impacts viral pathogenesis by altering viral replication, dissemination, or tropism to the brain. If the D355A mutant had defective dissemination or tropism to the brain, then we would expect to see wild type titers at the site of inoculation, as well as potentially the serum, but an absence of viral titer in the brain. Alternatively, if viral titers for the D355A mutant are significantly decreased in the ankle, serum, and brain, then this would suggest that the decreases seen in pathogenesis were due to poor viral replication and dissemination.

Surprisingly, in contrast to what was expected given our tissue culture data, viral titers in the ankle, serum, and brain were more or less equivalent between wild type SINV and the two nsP1 mutants (Figure 3.8). The similar titers found in the ankle between the nsP1 mutants and wild type SINV show that the reduced pathogenicity of the nsP1 D355A mutant is not due to a defect in viral replication at the site of inoculation (Fig. 3.8A). Likewise, since the nsP1 D355A mutant had titers equivalent to wild type levels in the serum, we can also conclude that viral dissemination was not negatively impacted (Fig. 3.8B). Finally, while the viral titer 
of the D355A and N376A mutant were both slightly decreased in the brain compared to wild type SINV, the lack of a significant difference indicates that increasing capping efficiency did not alter viral tropism to the CNS and that there is no overt defect in viral replication (Fig. 3.8C). Interestingly, while these results are different from what was previously observed during infection of BHK-21 cells (Figure 3.1D), the trends seen in the serum and brain, where the N376A mutant has slightly decreased viral titer compared to wild type SINV and the D355A mutant, was similar to what was seen with the L929 cells (Figure 3.5).

To further complement our observations regarding the induction of the host innate immune response to infection (as in Figure 3.4A), we quantitatively assessed IFN $\beta$ transcript levels in the ankles of the experimentally infected mice at $24 \mathrm{hpi}$. Despite differences in the overall magnitudes of type-I IFN induction, we found no differences between our in vitro and in vivo assessments (Supplemental Figure 3.1).

The dissemination of the SINV mutants to the brain was further confirmed when viral RNA levels in the brain were measured (Figure 3.8D). While the nsP1 D355A mutant did exhibit slightly lower vRNA abundance in the brain compared to wild type SINV, it was not found to be a statistically significant difference and is likely an artifact due to differences in when the brain tissue was collected. While the majority of the D355A infected mice survived to $7 \mathrm{dpi}$ when the brain tissue was collected, all of the wild type infected mice met endpoint criteria prior to $7 \mathrm{dpi}$. As such, the adaptive immune response in the D355A infected mice that survived to 7 dpi may have started to clear some of the infected cells serving as viral RNA 
reservoirs from the brain, resulting in the decreased vRNA abundance compared to the wild type infected mice that did not survive long enough to mount a similar response. N376A RNA levels in the brain were also found to be equivalent to that of wild type SINV. These results indicate that, while the D355A and N376A nsP1 point mutations were capable of reducing viral titer in tissue culture, they were not detrimental to viral replication in mouse models of infection.

Taken together, these data suggest that both the increased capping virus D355A and the decreased capping virus N376A were capable of trafficking to the brain from the site of inoculation, and were capable of replicating to high titers within the brains of experimentally infected adult mice. It is also interesting to note that neither viral titer nor viral genomic RNA abundance correlated with death, as there were multiple mice which survived infection that had greater viral titer and vRNA abundance in the brain than mice which died. Overall, these results led us to conclude that the attenuation of pathogenesis seen in the D355A mutant were not due to either deficits in viral replication or tropism.

\section{Increasing Capping Efficiency Does Not Affect Viral Induction Of Neuronal}

\section{Apoptosis.}

Since we did not find any significant differences in viral replication or tissue tropism / dissemination, we next determined if decreased mortality in the D355A mutant was due to an altered capacity to induce neuronal death, as virally induced apoptosis of neurons in the brain, brainstem, and spinal cord have been shown to be responsible for the severe neurological symptoms that arise during SINV 
infection [89, 179-181]. In addition, our previous study characterizing the nsp1 capping mutants in tissue culture showed that both the D355A and N376A mutant demonstrated increased cell viability in BHK cells compared to wild type SINV, supporting the possibility that the D355A mutant might have differences in cell viability in neurons as well [171]. In light of our previous study as well as the striking difference in cell death between the D355A and wild type infected brains (Figure 3.7), we hypothesized that increasing capping efficiency would decrease the virus' capacity to kill neurons. To test this hypothesis, we infected SK-N-BE(2) cells with either wild type SINV or one of the capping mutants and determined cell viability 24 hours after infection using ethidium bromide and acridine orange staining. Infection with the decreased capping N376A mutant resulted in significantly greater cell viability compared to either wild type SINV or the D355A mutant, which both exhibited roughly similar levels of cell death (Figure 3.9). Interestingly, these results suggest that increasing ncgRNA production leads to increased cell survival in tissue culture models of infection, while decreasing ncgRNA production does not seem to impact the virus' capacity to kill neurons in tissue culture models of infection. Given this, we can conclude that the differences seen in tissue damage between the D355A and wild-type SINV infections in Figure 3.7 are not solely due to deficits in the D355A mutant's capacity to kill neurons. Instead, the above results suggest that the neuronal death seen during SINV infection in mice may be largely due to the host antiviral inflammatory response rather than direct cell death due to infection. 


\section{Differential ncgRNA Expression Alters The Immune Response To Infection.}

Because the differences in morbidity and mortality between the D355A nsP1 mutant and wild type SINV infected mice could not be explained by reductions of viral titer or the capacity to induce neuronal death, we next questioned whether differences in pathogenesis could be due to an altered host immune response. Given the reduced immune infiltration and inflammation seen in the D355A infected mice compared to the wild type or N376A infected mice (Figure 3.7), we expected infection with the D355A mutant to also result in the decreased expression of pro-inflammatory genes. To survey the immune response to SINV nsp1 mutant virus infections, RNA was isolated from whole brain homogenates of infected mice at $7 \mathrm{dpi}$ or upon meeting endpoint criteria, and the level of select cytokine and chemokine transcripts were measured via a qRT-PCR based array. Out of the transcripts measured, 50 were found to be significantly increased in wild type infected versus mock infected mice (Figure 3.10A). When wild type and D355A infections were compared, we found 15 inflammatory cytokines and chemokines whose expression was determined to be significantly decreased (using a Benjamini-Hochberg corrected $p$-Value) by a magnitude greater than 2-fold (Figure 3.10B). Individual box and whisker plots of these statistically significant transcripts may be found in Fig. 3.10C. These included chemokines involved in recruiting innate immune cells, such as CCL2, CCL3, and CXCL10, as well as important drivers of inflammation like IFN $\beta$, IL1 $\beta$, TNFa. In particular, the expression of CCL2 and CCL3 have been highly correlated with areas of the brain experiencing high levels of gliosis and apoptosis, which is 
consistent with the differences in pathology scoring in those areas between the D355A mutant and wild type SINV (Figure 3.7B) [182]. Interestingly, several of the proteins that had decreased expression in the D355A infected mice compared to wild type infected mice where identified by gene ontology as being part of the extrinsic apoptotic pathway $[183,184]$. The identification of proteins involved in apoptosis, specifically Fas, $\|1 \alpha\| ,1 \beta$, and TNF $\alpha$, is consistent with the significant decrease in neuronal apoptosis seen in the H\&E stained sections of mice infected with the D355A mutant. Surprisingly, IFNy expression, which has been previously shown to be important for noncytolytic clearance of virus from neuronal cells, was not found to be significantly different in either mutant compared to wild type (Figure 3.10A) [185]. As expected, the decreases seen in the expression of these chemokines and pro-inflammatory cytokines are consistent with the reduced immune infiltration and tissue damage seen in Figure 3.7.

Overall, these data show that the D355A increased capping mutant has significantly reduced pathogenicity in a wild type mouse model of infection correlating with decreased expression of multiple pro-inflammatory molecules at the transcriptional level. Furthermore, the above data suggests that it is likely the host response rather than viral replication per se that determines the extent of alphaviral pathogenesis. This is supported by the finding that the wild type and D355A viruses both had roughly equivalent viral titers in the brain, yet wild type SINV had significantly increased pro-inflammatory cytokine transcript expression compared to the D355A mutant. While transcript levels are not synonymous with protein levels, the correlation between the expression of antiviral transcripts and 
levels of inflammation in the brain suggest that antiviral protein expression likely follows the same trends. Taken together, it can be concluded that decreased ncgRNA production via increased capping efficiency leads to an altered host immune response, which in turn shapes alphaviral pathogenicity.

\section{Discussion}

\section{Altering Capping Efficiency Changes Viral Sensitivity To And Activation Of Host Type-I IFN.}

For Sindbis virus, the sensitivity of the virus to type-I IFN is largely dependent on the translation of the nonstructural proteins which are responsible for interfering with the IFN signaling pathway and for shutting down host transcription [174, 186-188]. When capping efficiency was increased with the D355A mutant, nonstructural gene expression was also found to be increased, although the magnitude was not as drastic as was previously seen with the Toto1101-derived background (Figure 3.2). However, this may not be necessarily representative of what happens early during viral infection where the magnitude of effect is likely to be more pronounced. While we were previously able to characterize early viral translation in the Toto1101-derived background using a nanoluciferase reporter incorporated into nsP3, the construction of the identical reporter in the AR86 background resulted in significant attenuation. However, given that we have previously shown that the differences in viral translation for the D355A mutant were more pronounced early during infection, and that increasing capping was correlated with increased genomic translation in both viral 
backgrounds (Fig. 3.2C), it is likely that the D355A mutant in the AR86 background would also follow this trend [171]. Increased translation of the viral proteins would allow the virus to more quickly shut down IFN signaling pathways, host PAMP sensors, as well as host transcription and translation, which would explain why the D355A mutant showed reduced IFN $\beta$ and ISG expression and greater resistance to IFN $\beta$ treatment $[175,189-191]$. In addition, the altered IFN $\beta$ and ISG expression seen with the capping mutant likely reflects changes in the virus' abilities to both avoid detection by the host as well as suppress the cellular antiviral response [186]. Because IFN $\beta$ and ISG transcript abundance was normalized to viral RNA levels, we know that the differences seen in antiviral transcript expression are not simply due to differences in viral replication or the amount of vRNA present. Rather, the changes observed in IFN $\beta$ and ISG expression for the D355A and N376A nsP1 mutants are likely due to both differences in PAMP production and their abilities to shutoff host transcription and translation. This is supported by the correlation between ncgRNA production, which are a viral PAMP, and IFN $/$ /ISG expression (Fig. 3.1B and Fig. 3.4) [189, 190]. Also, the fact that the D355A mutant showed increased resistance to type-I IFN even when IFN was added concurrently with the virus demonstrates how increasing capping efficiency allows the virus to more readily mitigate the effects of the IFN response. The D355A mutant's increased resistance to IFN at such an early timepoint suggests that, in addition to increased translation, the mutation may also affect how quickly the virus progresses through the viral life cycle. Increasing the production of capped genomic vRNA could potentially give the virus a head start on translation of the 
viral proteins responsible for shutting down the host IFN response, allowing the D355A mutant to be more resistant to IFN treatment early during infection.

\section{Noncapped Genomic vRNAs Are Critical For SINV Pathogenesis In Mice.}

The ability to resist and shut down the type I IFN response has been shown to be one of the major determinants of virulence for SINV. For example, the AR86 strain of SINV is a virulent strain known to efficiently suppress the type I IFN response and cause lethal neurotropic disease in adult mice. The genetically similar Girdwood strain only partially inhibits the type I IFN response, and thus is avirulent in adult mice [101]. Given the above finding that the D355A mutant was both more resistant to type I IFN treatment and resulted in decreased ISG production compared to wild type virus, we were surprised to find that this mutant did not cause severe disease or mortality in mice. Equally surprising was the result that infection with the N376A mutant was similarly as severe and lethal as infection with wild type SINV, despite N376A being more sensitive to IFN treatment and stimulating more ISG expression in vitro. Taken together, this suggests that there may be a balance between inhibiting and activating the IFN response which results in pathogenesis, and that tipping the scales too far in either direction causes the virus to become avirulent. Furthermore, our results indicate that ncgRNA play a critical role in determining whether the virus is neurovirulent or avirulent, as decreasing ncgRNA production with the D355A mutation resulted in significant decreases in morbidity and mortality while increasing ncgRNA production with the N376A mutation resulted in fully neurovirulent virus and lethal disease. 
Although the D355A mutation had a much more striking impact on morbidity and mortality, the N376A mutation also had noticeable impacts on alphaviral pathogenesis, namely a delay in the onset of symptoms, an increased mean survival time, and a moderate increase in overall survival compared to wild type SINV. The idea that inhibiting capping efficiency is detrimental to alphaviral infection is not novel and multiple compounds and drugs have been developed to specifically target nsP1 capping activity [166-169]. Our results do not negate the idea that decreasing or inhibiting capping efficiency is an effective means of combating alphaviral infection, but rather suggest that there is a threshold which needs to be reached before decreasing capping efficiency will be significantly detrimental to viral pathogenesis. Likewise, our mortality studies imply that the virus is much more sensitive to increasing capping efficiency and that this novel approach may be more effective in limiting the severity of viral disease.

During SINV infection, the development of severe encephalitis which leads to paralysis and death is caused by the extensive apoptosis and necrosis of neurons in the brain and CNS, which is believed to be virally induced [86, 179 , 181, 192]. While apoptosis is the fate of many infected cells and neurons, a significant portion of uninfected neurons are killed during viral infection due to glutamate excitotoxicity [87]. The lack of apoptosis and necrosis seen in the brains of mice infected with the D355A mutant (Fig. 3.7) suggests that, during in vivo infection, decreasing ncgRNA production by increasing nsP1 capping efficiency leads to decreased viral induction of neuronal apoptosis and therefore decreased glutamate release, resulting in the reduced death of infected and uninfected 
neurons. However, when tested in tissue culture cells, infection with the nsP1 D355A mutant resulted in wild type levels of neuronal death, while infection with the N376A mutant, which showed extensive signs of neuronal apoptosis and necrosis in vivo, resulted in significantly increased neuron survival. Since increasing capping efficiency did not seem to impact the virus' ability to induce neuron death in tissue culture, these data suggest that the apoptosis and necrosis of neurons seen in the brain during SINV infection is largely mediated by an external force, such as by the host immune response. This is supported by previous studies which propose that the majority of neuronal death due to apoptosis and glutamate excitotoxicity may be the work of $\mathrm{T}$ cells and astrocytes, rather than being directly virus-induced [193, 194].

Another surprising result was that disease severity did not correlate with increased viral titer or vRNA abundance. This is most clearly seen in the N376A infected mice, where the viral titers and vRNA levels in the brains of mice that died are interspersed among those which survived. In addition, surviving mice from both the D355A and N376A infections had titers and vRNA levels in the brain that were roughly equivalent to those found in the mice infected with wild type SINV which died. Furthermore, neither viral titer nor vRNA burden in the brain correlated with levels of inflammation seen in Figure 3.7. This suggests that, during SINV infection, high viral titer alone is not sufficient to cause severe disease in mice. In addition, altering capping efficiency did not significantly impact viral replication, dissemination, or tropism. This was illustrated by the roughly wild type-equivalent titers found in the ankle, serum, and brain indicating that both of the capping 
mutants were able to efficiently replicate at the site of inoculation, disseminate into the blood, and traffic to the brain. However, the slight decrease in N376A titer seen in the blood and brain does potentially suggest that dissemination may be slightly delayed or impaired when capping efficiency is decreased, and may explain the slight increase seen in mean survival time.

Unfortunately, one question we were unable to answer in this study was whether viral capping efficiency in brain tissue was similarly affected by the nsP1 mutations, as was previously shown in tissue culture model systems and with recombinant proteins $[124,171]$. Regrettably, the limitations in sensitivity of previously established and currently available assays render us unable to directly answer this question, as these methods require a significant quantity of highquality viral RNA that is difficult to obtain from brain tissue. This is likely due to the fact that an exceptionally few number of cells in the brain are required to be infected for the manifestation of significant disease and the appearance of endpoint criteria. However, the altered pathogenesis seen in mice in the absence of any obvious defects in viral replication, dissemination, or tropism lead us to believe that the point mutations incorporated are still altering capping efficiency and are not significantly affecting nsP functions in other ways. Previously characterized mutations in nsP1 which resulted in loss of neurovirulence did so by significantly altering vRNA synthesis and/or processing of the nonstructural polyprotein, which typically resulted in decreased viral titer in animal models of infection $[100,101,170,195]$. Given that neither the D355A nor N376A mutations significantly altered viral titer or vRNA burden during SINV infection in vivo, nor 
negatively impacted vRNA synthesis or viral translation in vitro, we can conclude that the phenotypes seen both in tissue culture and in animal models of infection are the result of the mutations altering ncgRNA production through modulating capping efficiency. Furthermore, given the conserved effect of the D355A and N376A nsP1 point mutations in multiple alphaviruses in tissue culture and in vitro, it is likely that these mutations still respectively increase or decrease capping efficiency in vivo, but the magnitude that capping efficiency is altered may be different from what was previously seen in tissue culture [124, 171].

\section{Noncapped Genomic RNAs Determine SINV Virulence by Modulating the Host Inflammatory Response.}

Infection with the D355A capping mutant resulted in the decreased expression of multiple cytokines and chemokines associated with the recruitment of immune cells, regulating inflammation, and apoptosis. While there were a small number of cytokines found to be differentially expressed during infection with the N376A mutant compared to wild type SINV, they did not implicate any pathways in particular. Interestingly, expression of anti-inflammatory transcripts such as TGF $\beta$ and IL-10 was found to be similar between D355A and wild type infection, while others such as SOCS1 were found to be significantly decreased. This suggests that the decreased inflammation seen with the D355A mutant is due to decreased activation of antiviral and inflammatory pathways rather than increased expression of anti-inflammatory cytokines. The decreased activation of these antiviral pathways are likely due to both the reduced release of DAMPS from dying 
cells and decreased sensing of viral PAMPS. The first is supported by the identification of several of the affected proteins being involved in apoptosis as well as the decreased level of cell death seen with the D355A mutant. The second is supported by the decreased IFN $\beta$ and ISG expression seen during D355A infection (Figure 3.4). The decreased sensing of viral PAMPS may be due to the D355A mutant either being more efficient at inhibiting the cell's viral sensors and signaling pathways through shutoff of host transcription or could be due to the D355A mutant producing fewer noncapped RNA, which are an established PAMP [189, 190]. Given that viral infection in animals is a continuous process, shut-off of cellular transcription and suppression of the IFN response in tissues likely does not occur as efficiently or completely as it does in cell culture, where all the cells are infected simultaneously. Therefore, the decreased production of inflammatory cytokines seen with the D355A mutant is likely due to reduced detection of DAMPs and PAMPs caused by decreased cell death and decreased production of ncgRNA. How exactly the ncgRNA are sensed by the host during viral infection is not currently known and is an ongoing interest in the Sokoloski lab. While there is some evidence that suggests that the noncapped RNA produced during alphaviral infection are at least in part sensed by RIG-I, it is unknown if this is also true for ncgRNA and there may be additional methods for detecting noncapped vRNA that have yet to be characterized $[190,196]$. Overall, the correlation between decreased inflammation and decreased ncgRNA production leads us to conclude that the ncgRNA play a critical role in determining the host response to viral infection. 
In conclusion, we have identified a novel determinant of Sindbis virulence which operates through a separate mechanism than those previously described. Specifically, decreasing the production of ncgRNA by increasing capping efficiency results in the loss of neurovirulence which we believe is due to the reduced production of RNA PAMPs that would otherwise cause excess inflammation and wide-spread cell death in the brain (Fig. 3.11). The D355A mutation differs from previously identified nsP1 virulence determinants in that it does not negatively affect viral titer nor resistance to IFN, such as is seen with the SINV nsP1 cleavage mutant T538I and the 6 nsP1 mutations characterized in Ross River virus $[97,170]$. The D355 residue in nsP1 is also unique from the aforementioned mutation sites in that it is very highly conserved among SINV strains as well as across both the old and new world alphaviruses. While the results of this paper indicate that the production of ncgRNA is critical to SINV pathogenesis, more work is needed to further characterize the mechanisms by which ncgRNA contribute to alphaviral neurovirulence. 
$\Delta \quad \begin{gathered}\text { SINV AR86 nsP1 Mutant } \\ \text { Genomic RNA 5' Characterization }\end{gathered}$

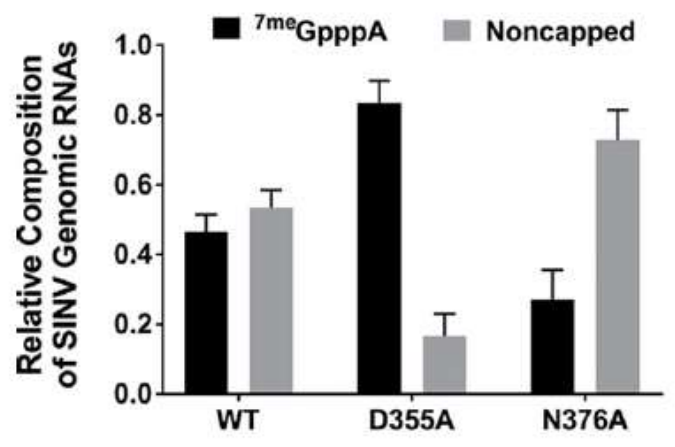

SINV AR86 nsP1 Mutant

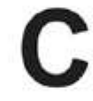

Relative Difference in Production of Capped Genomic RNAs

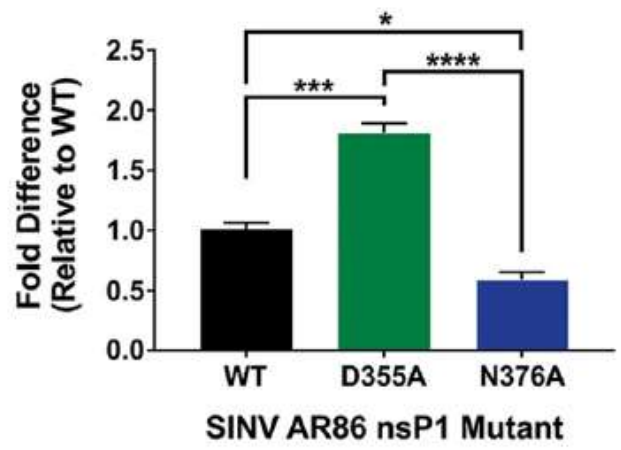

Relative Difference in Production of Noncapped Genomic RNAs

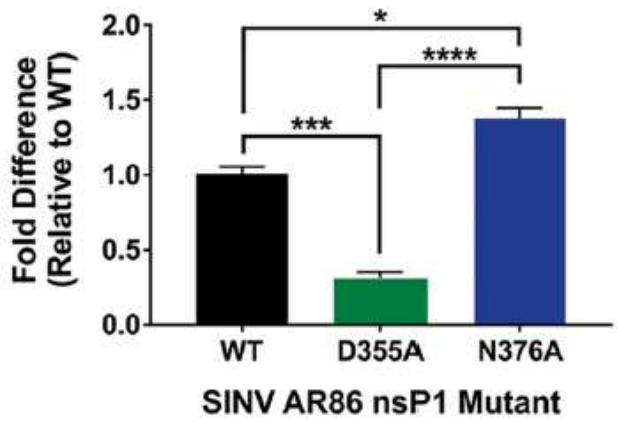

AR86 SINV nsP1 Mutant Mammalian Growth Kinetics

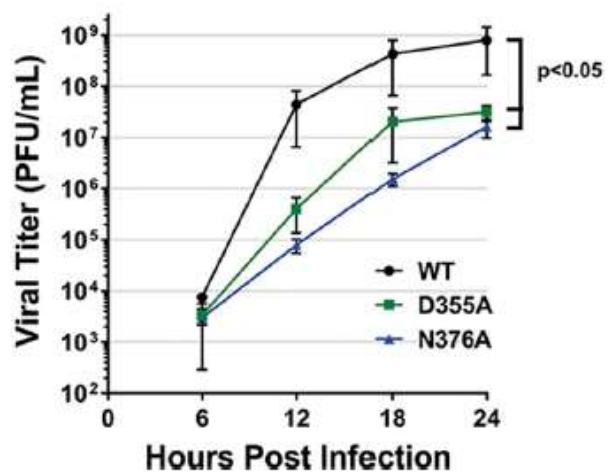

Figure 3.1. Point mutations in nsP1 of AR86 SINV result in changes in capping efficiency and negatively impacts infection in mammalian cells. (A) Quantitative assessment of SINV RNAs produced during infection of BHK-21 cells with either wild type SINV or either of the nsP1 mutants at an MOI of 5 PFU/cell. RNA was collected at 16hpi and treated as described in Materials and Methods. Graphs depicting the relative quantities of noncapped (B) or capped (C) genomic RNAs produced during infection of BHK-21 cells with either wild type SINV or either of the nsP1 mutants at 16hpi. (D)One-step growth kinetics of the individual capping mutants and the parental wild-type SINV in BHK-21 cells infected at an $\mathrm{MOI}$ of $5 \mathrm{PFU} / c e l l$. All the quantitative data shown represent 
means of results from three independent biological replicates, with error bars representing standard deviation of the means. Statistical significance was determined by analysis of the area under the curve. ${ }^{*}=p<0.05 .{ }^{* *}=p<0.01$. ${ }^{* * *}=p<0.001 .{ }^{* * * *}=p<0.0001$. 

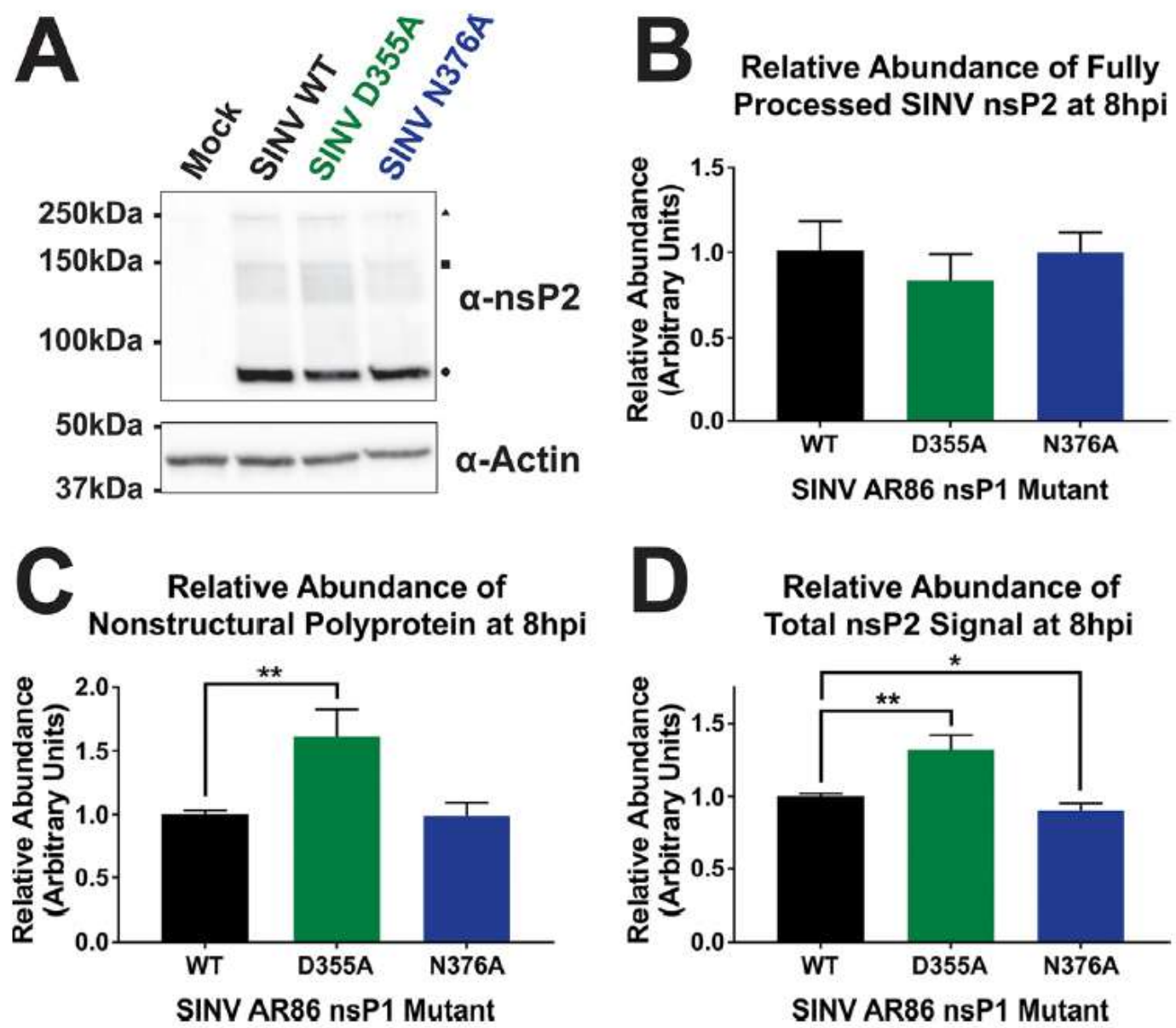

Figure 3.2. Increasing capping efficiency increases translation of SINV AR86 nonstructural polyprotein. (A) BHK-21 cells were infected with either wild type SINV or one of the nsP1 capping mutants at an MOI of $5 \mathrm{PFU} / c e l l$. Abundance of nsP2 was then assessed at 8hpi by Western blotting. Nonstructural polyprotein band marked by $\boldsymbol{\Lambda}$. The p23 polyprotein intermediate band (as determined by molecular weight) is marked by $\mathbf{\square}$. Fully processed nsP2 band marked by $\bullet$. Actin is shown as the loading control. Densitometric quantification of fully processed nsP2 protein (B), nonstructural polyprotein (C), and total nsP2 signal (D) normalized to actin levels at 8hpi. All the quantitative 
data shown represent means of results from three independent biological replicates, with error bars representing standard deviation of the means. Statistical significance was determined using Student's t-test. 

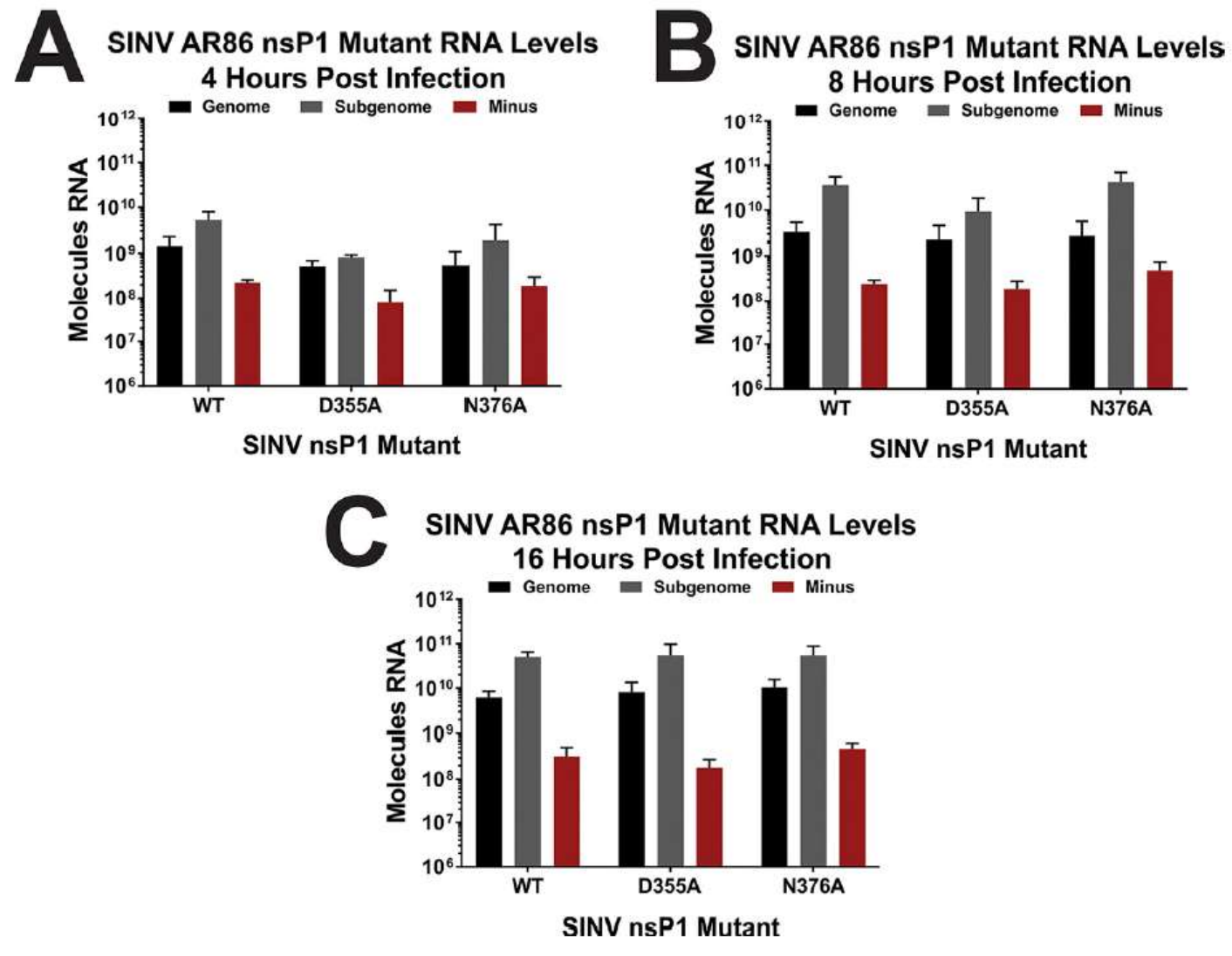

Figure 3.3. Altering capping efficiency does not impact AR86 SINV vRNA synthesis. BHK-21 cells were infected with either wild type SINV or one of the nsP1 capping mutants at an MOI of $5 \mathrm{PFU} /$ cell. Absolute quantities of the genomic, subgenomic, and minus strand vRNAs produced at $4(A), 8(B)$, and 16hpi $(C)$ were determined by qRT-PCR. All the quantitative data shown represent means of results from three independent biological replicates, with error bars representing standard deviation of the means. Statistical significance was determined using Student's t-test. 


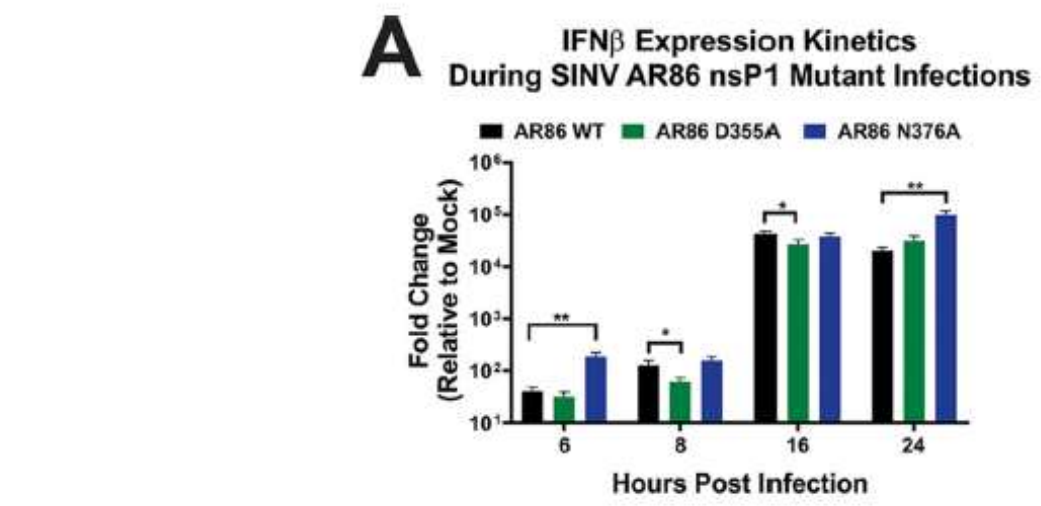

B

Interferon Stimulated Gene Expression 6 Hours Post Infection - AR86 WT AR86 D355A $=$ AR86 N376A
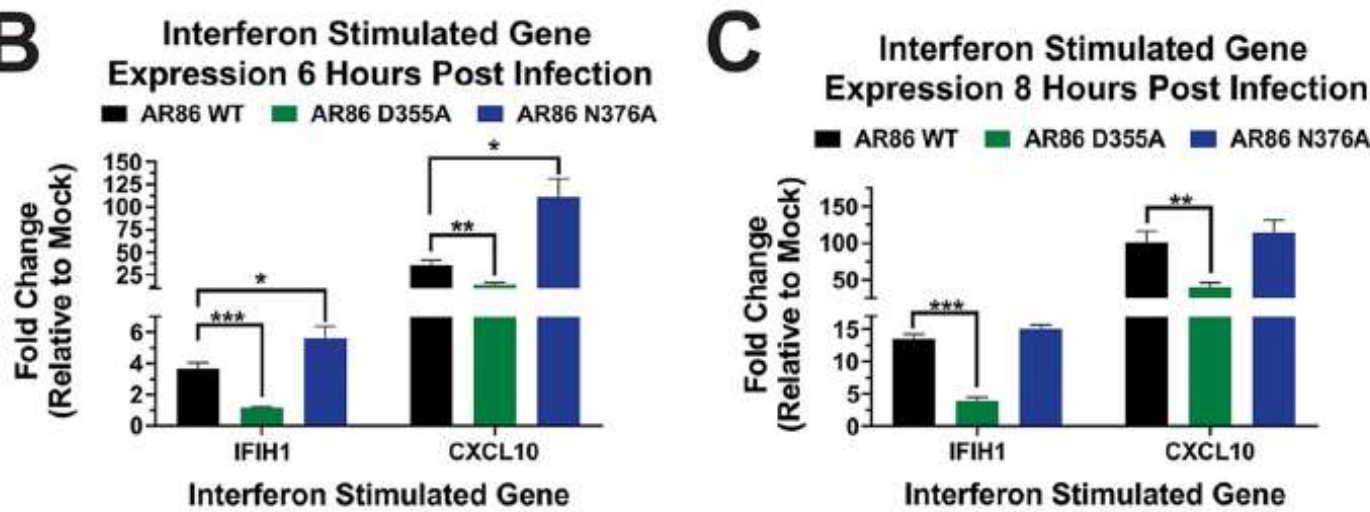

D

Interferon Stimulated Gene Expression 16 Hours Post Infection

- AR86 WT $=$ AR86 D355A AR86 N376A
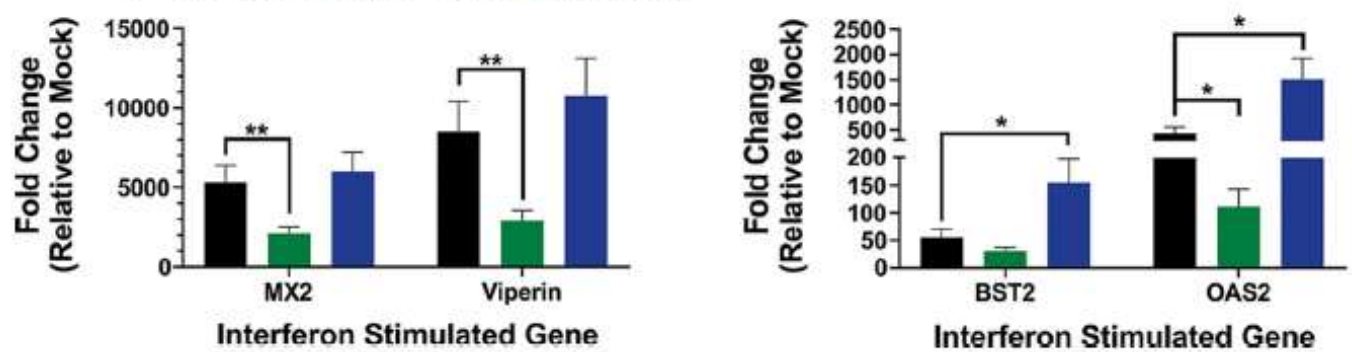

Figure 3.4. Production of type-I interferon and ISGs in response to SINV nsP1 capping mutants. L929 cells were infected at an MOI of $10 \mathrm{PFU} / \mathrm{Cell}$ with either wild-type SINV or an individual capping mutant. Cell lysates were collected at $6,8,16$, and $24 \mathrm{hpi}$ and transcript expression levels for IFN $\beta(A)$ and the selected ISGs (B-E) were determined by qRT-PCR for their respective times post infection. Data was normalized to GAPDH and nsP1 and calculated relative to 
uninfected controls. All the quantitative data shown represent means of results from three independent biological replicates, with error bars representing standard deviation of the means. Statistical significance was determined by Student's $t$ test. 

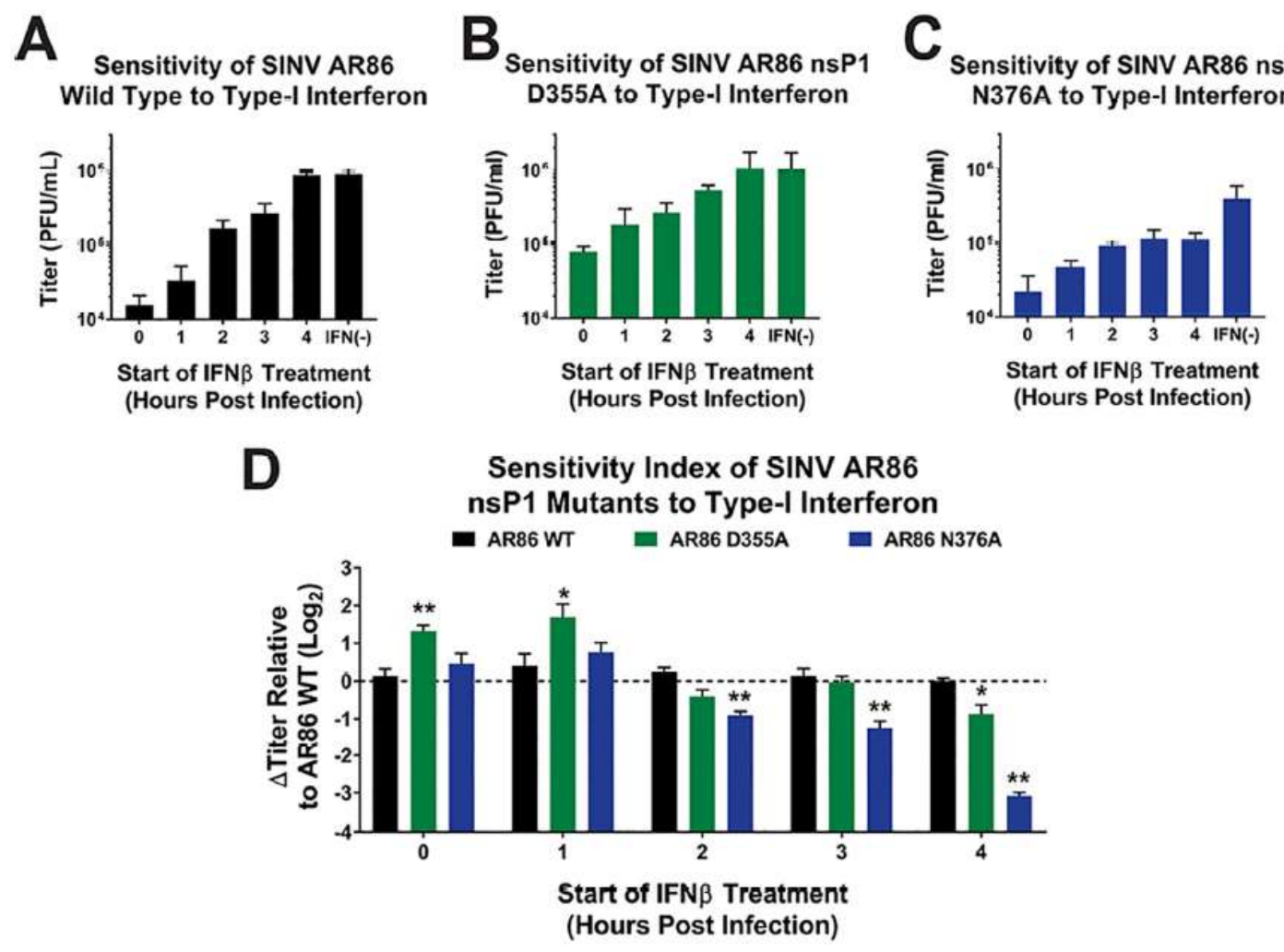

Figure 3.5. Analysis of SINV sensitivity to type-I interferon. L929 cells were infected with either wild-type SINV or an individual capping mutant at an MOI of 10 PFU/cell. At the indicated times post infection, 20 IU of recombinant Type-I IFN was added to the growth medium and the cells were incubated for a period of 24 hours. (A-C) Viral titers were quantified via plaque assay, and (D) the relative sensitivity of the viruses was determined by comparing their growth relative to untreated controls. All the quantitative data shown represent means of results from three independent biological replicates, with error bars representing standard deviation of the means. Statistical significance was determined by Student's $t$ test. ${ }^{*}=p<0.05 .{ }^{* *}=p<0.01$. 
A

SINV nsP1 Mutant Infected Animal Weights

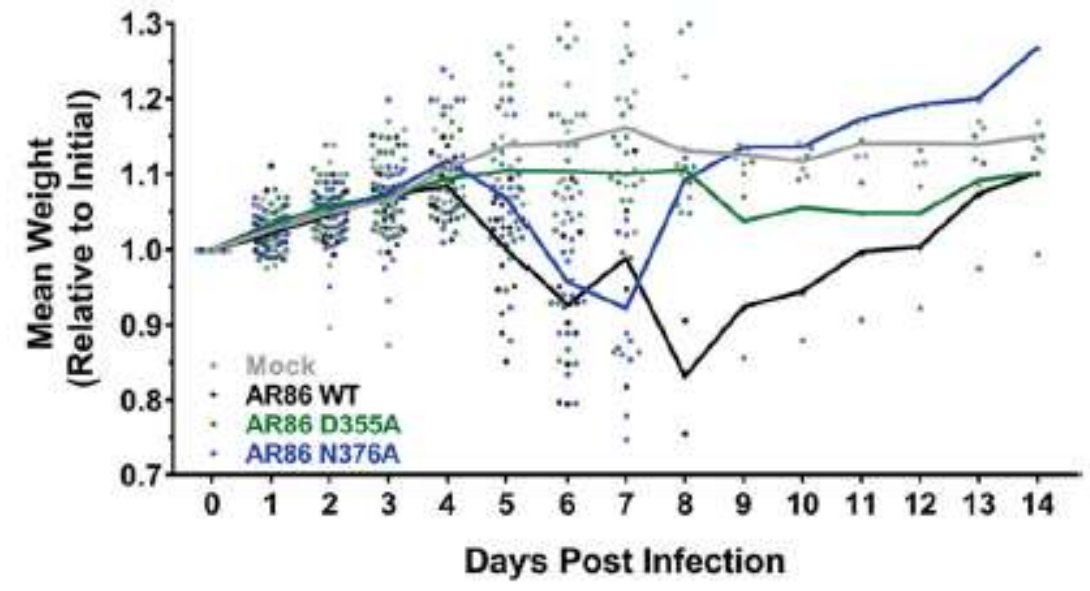

B SINV nsP1 Mutant Neurological Clinical Scoring

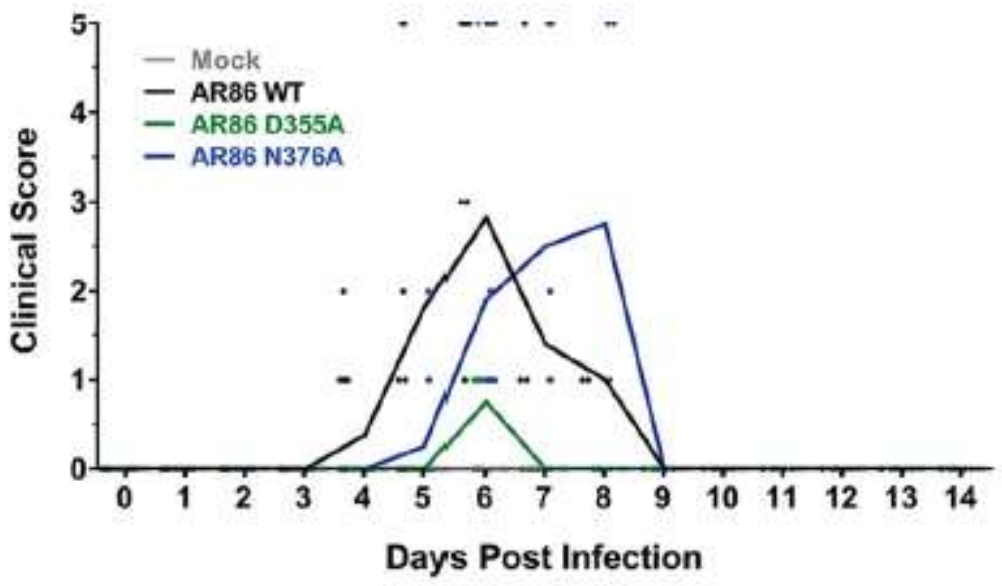

\section{SINV nsP1 Mutant Survival Curves}

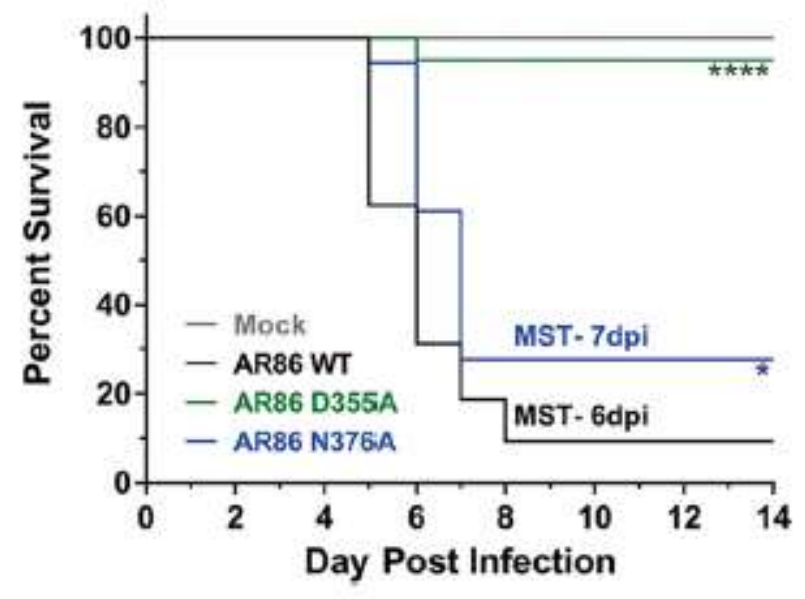


Figure 3.6. Increased vRNA capping efficiency reduces SINV AR86 mortality and pathogenesis. 4-week old male and female C57BL/6J mice were either mock infected or infected with 1,000 PFU of SINV AR86 wild-type, D355A, or N376A via rear footpad subcutaneous inoculation. Each data point represents a single animal from either experimental replicates. The experimentally infected mice were assessed over a 14 day period. (A) Animals were weighed twice daily. Weights are shown relative to initial weight after being infected. (B) Mice were scored based on a 1-5 scale for neurological response. (C) Kaplan-Meier analysis indicates the WT median survival time (MST) at $\sim 6.4$ days and the N376A mutant MST at $\sim 7$ days. The $P$ values indicated on the figure were determined by the Log-rank test. ${ }^{*}=p<0.05, \quad{ }^{* * * *}=p<0.0001$. Data shown were pooled from 2 independent experiments with an $\mathrm{n}$ of at least 16 per experiment. 

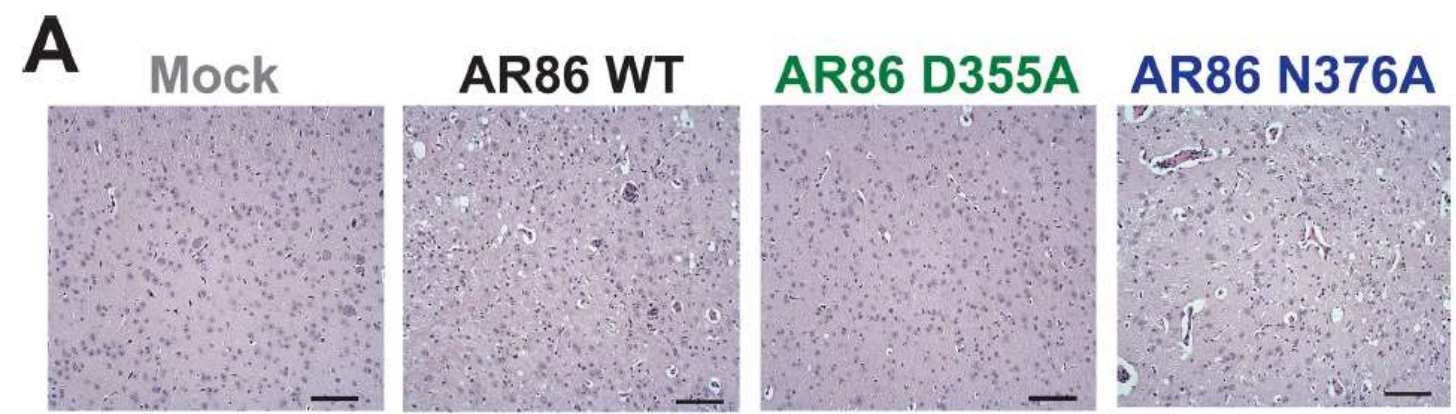

B
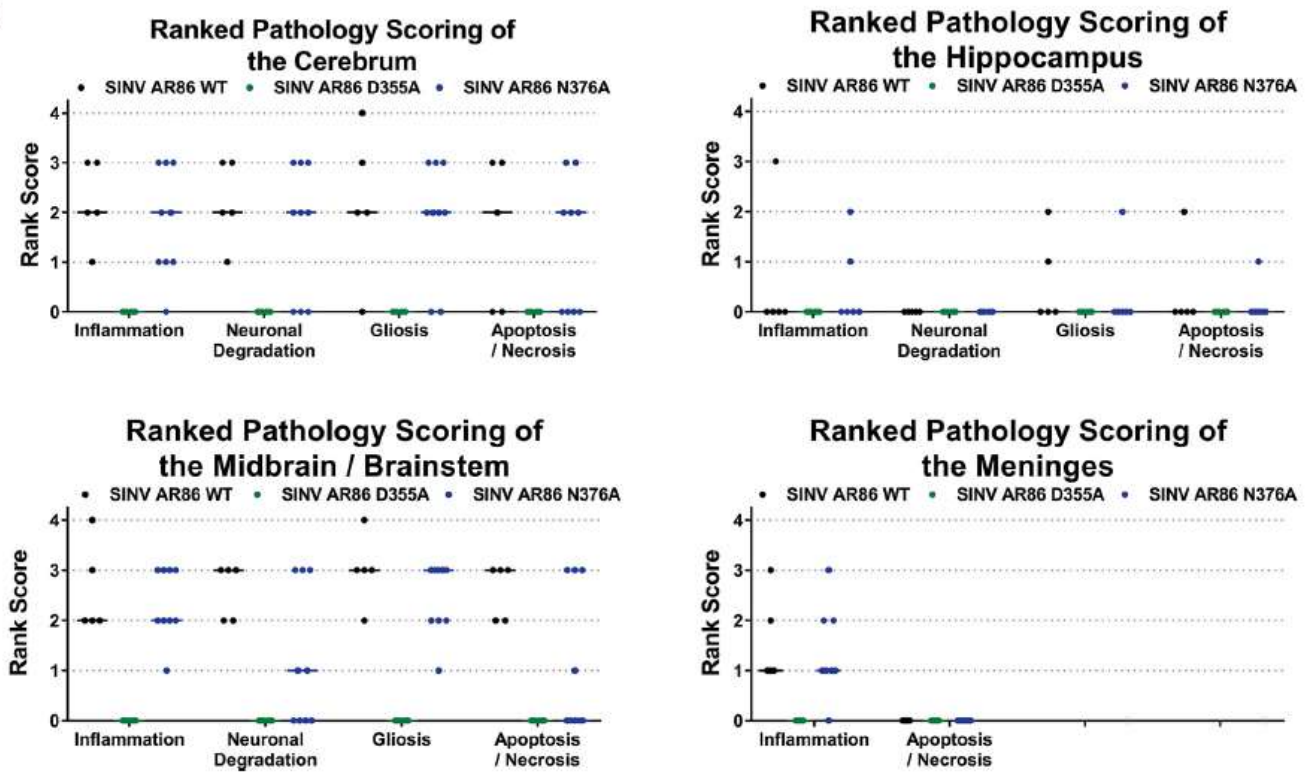

Figure 3.7. Increased capping efficiency leads to decreased pathology in the brain. A) Representative H\&E stained sagittal sections of the midbrain (20x magnification) from mock, wild-type, or capping mutant infected mice at $7 \mathrm{dpi}$ or at the time at which end point criteria was met. The brains of SINV wild-type and N376A infected mice show large amounts of perivascular cuffing, immune infiltration, and cell death not present in the mock and D355A infected mice. Scale bar $=0.1 \mathrm{~mm}$. B) Ranked pathology scoring of indicated sections of the brain from infected mice. Data points indicate scoring for each experimental animal, representing at least 5 biological replicates. 


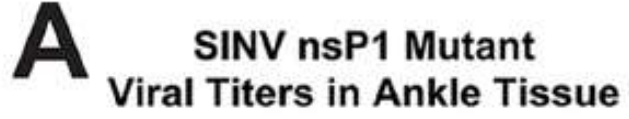

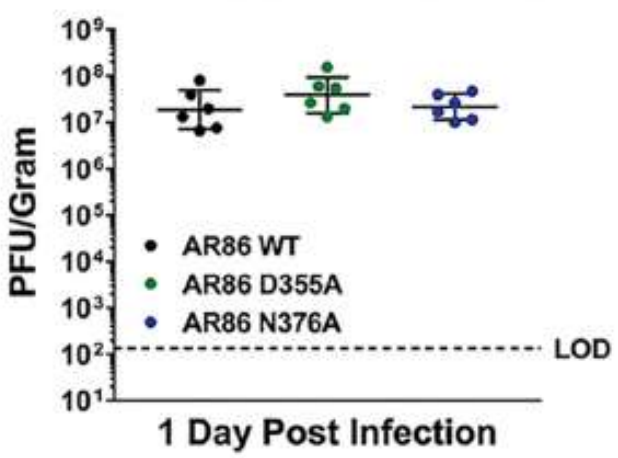

C

SINV nsP1 Mutant Viral Titers in Brain Tissue

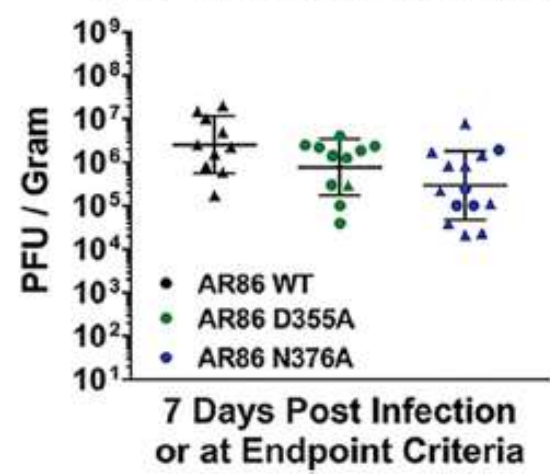

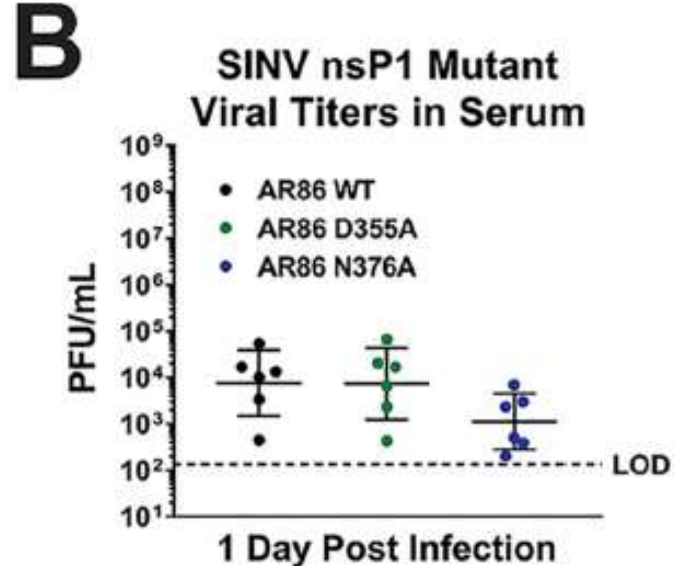

1 Day Post Infection

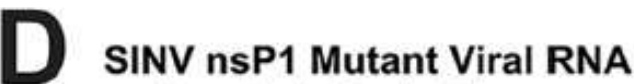
Levels in Brain Tissue

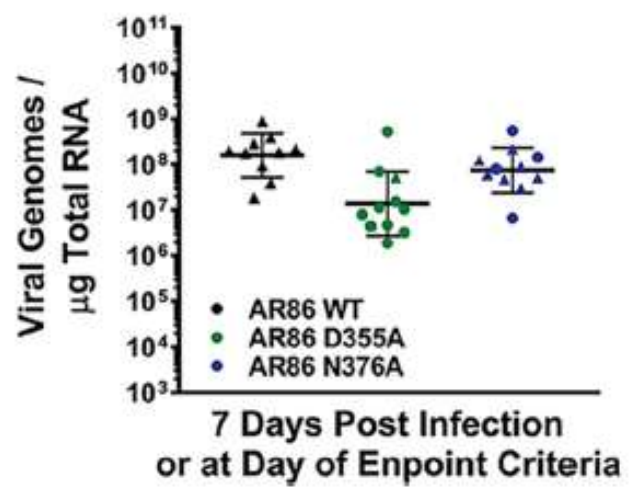

Figure 3.8. Viral replication is largely unaffected by altered capping in vivo. (A-C) Tissues were harvested at the indicated times post infection and viral titer was determined via plaque assay. (D) Viral genomes were measured by qRTPCR. The data points indicate the individual titers for each experimental animal, and the mean values shown are the geometric means of at least four biological replicates from two independent experiments, with the error bars representing the geometric standard deviations of the means. $\mathbf{\Delta}=$ mice that met endpoint criteria prior to day 7. Statistical significance was determined using Student's t test. 


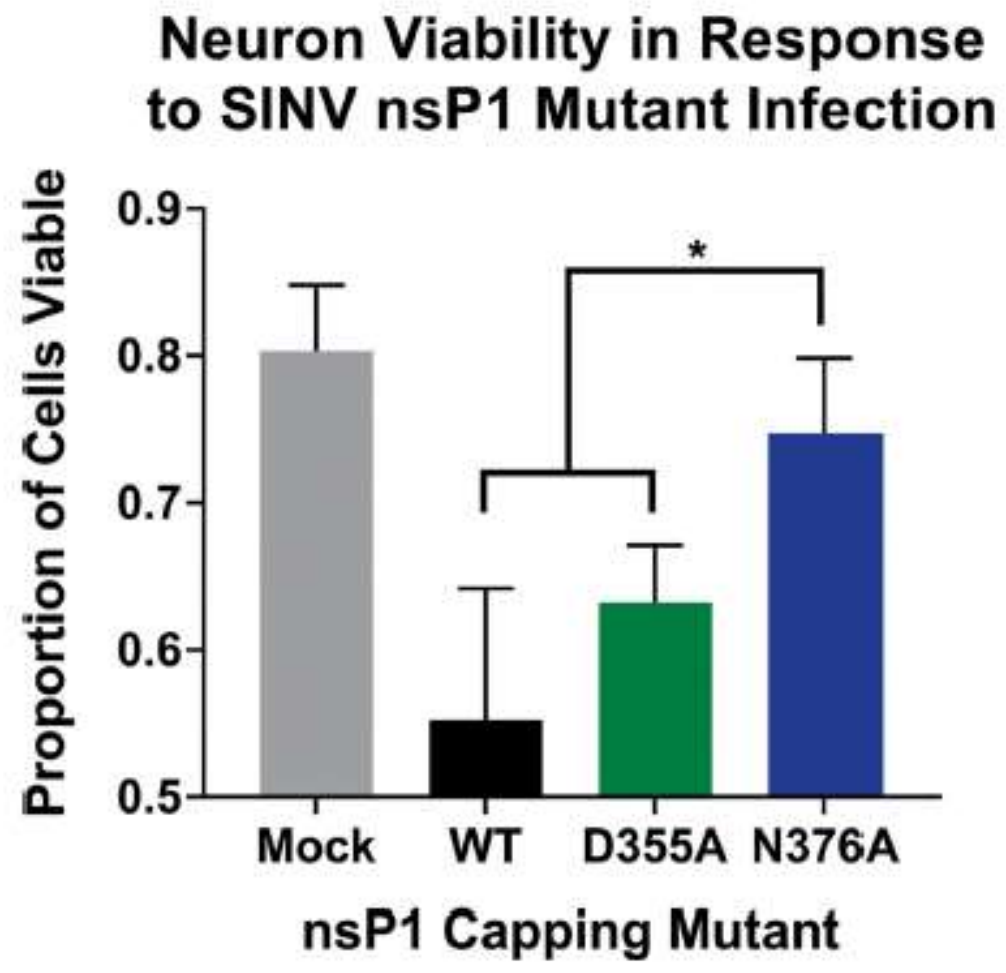

Figure 3.9. Neuron viability increased with decreased capping efficiency. SK$\mathrm{N}-\mathrm{BE}(2)$ neurons were infected at an MOI of $30 \mathrm{PFU} / \mathrm{Cell}$ with either wild-type SINV or an individual capping mutant. Cell viability was determined at $24 \mathrm{hpi}$ using ethidium bromide/ acridine orange staining and is represented as the proportion of viable cells out of total cells counted. A minimum of 100 total cells per well were counted using ImageJ. All the quantitative data shown represent means of results from three independent biological replicates, with error bars representing standard deviation of the means. Statistical significance was determined by Student's $t$ test. 


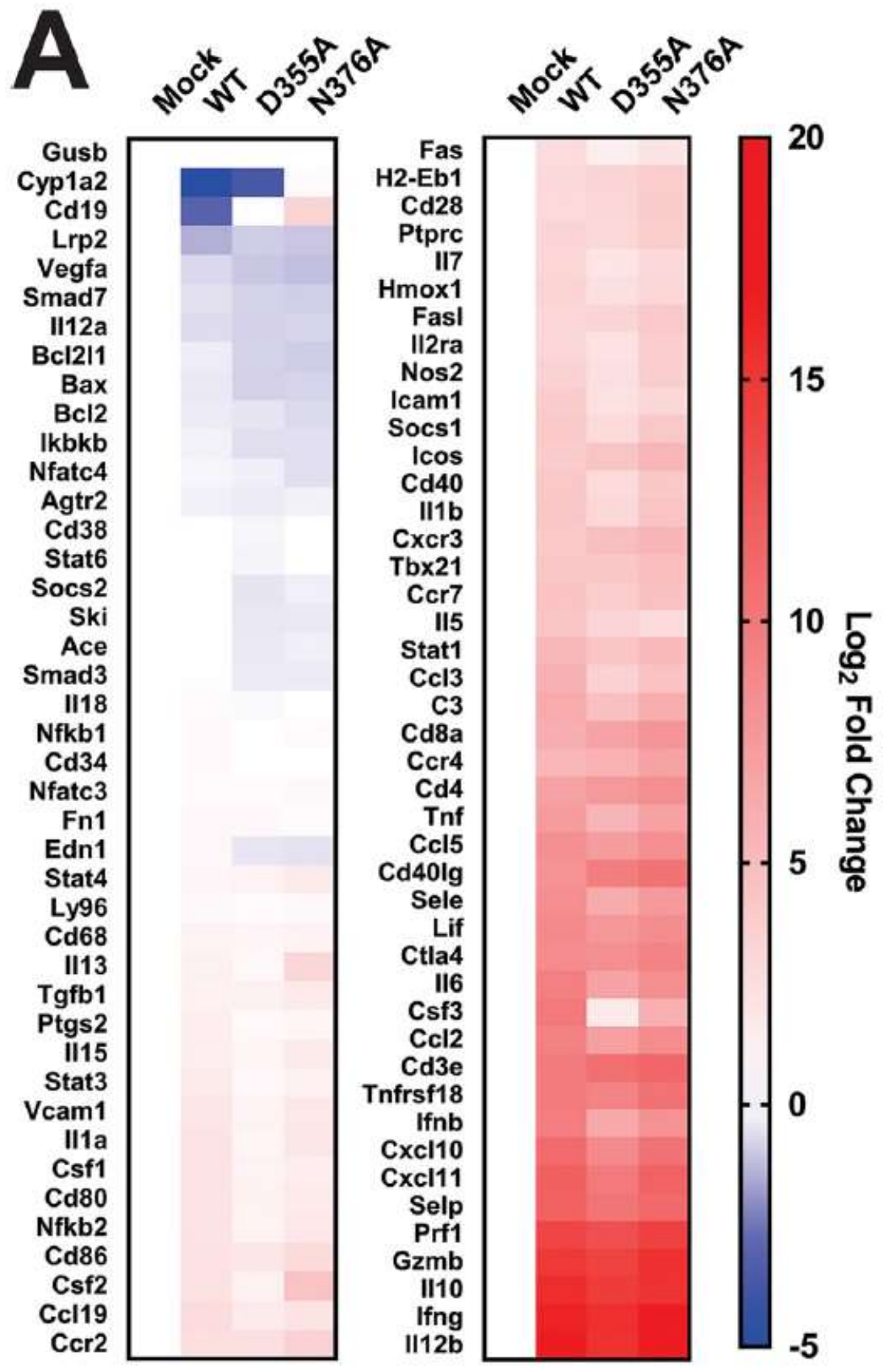


B Cytokine Transcript Analysis of SINV AR86 WT and SINV AR86 D355A

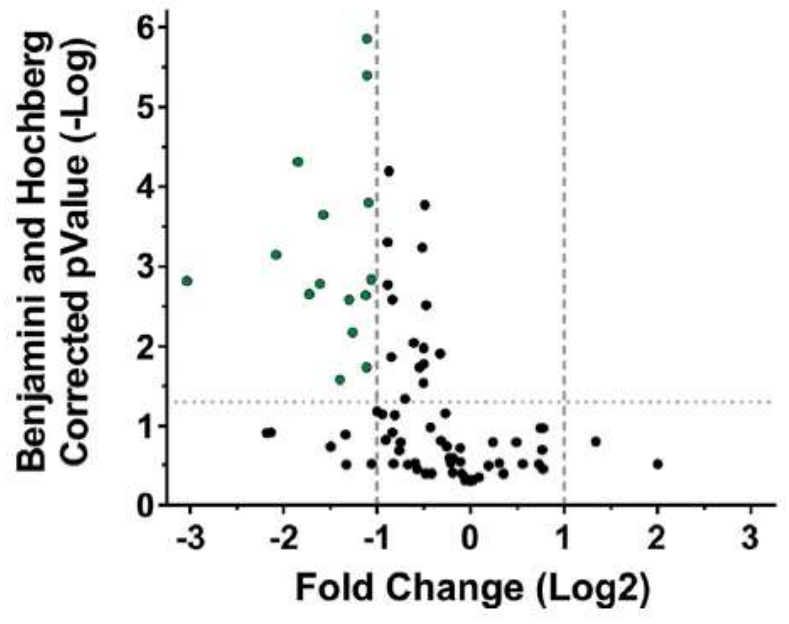



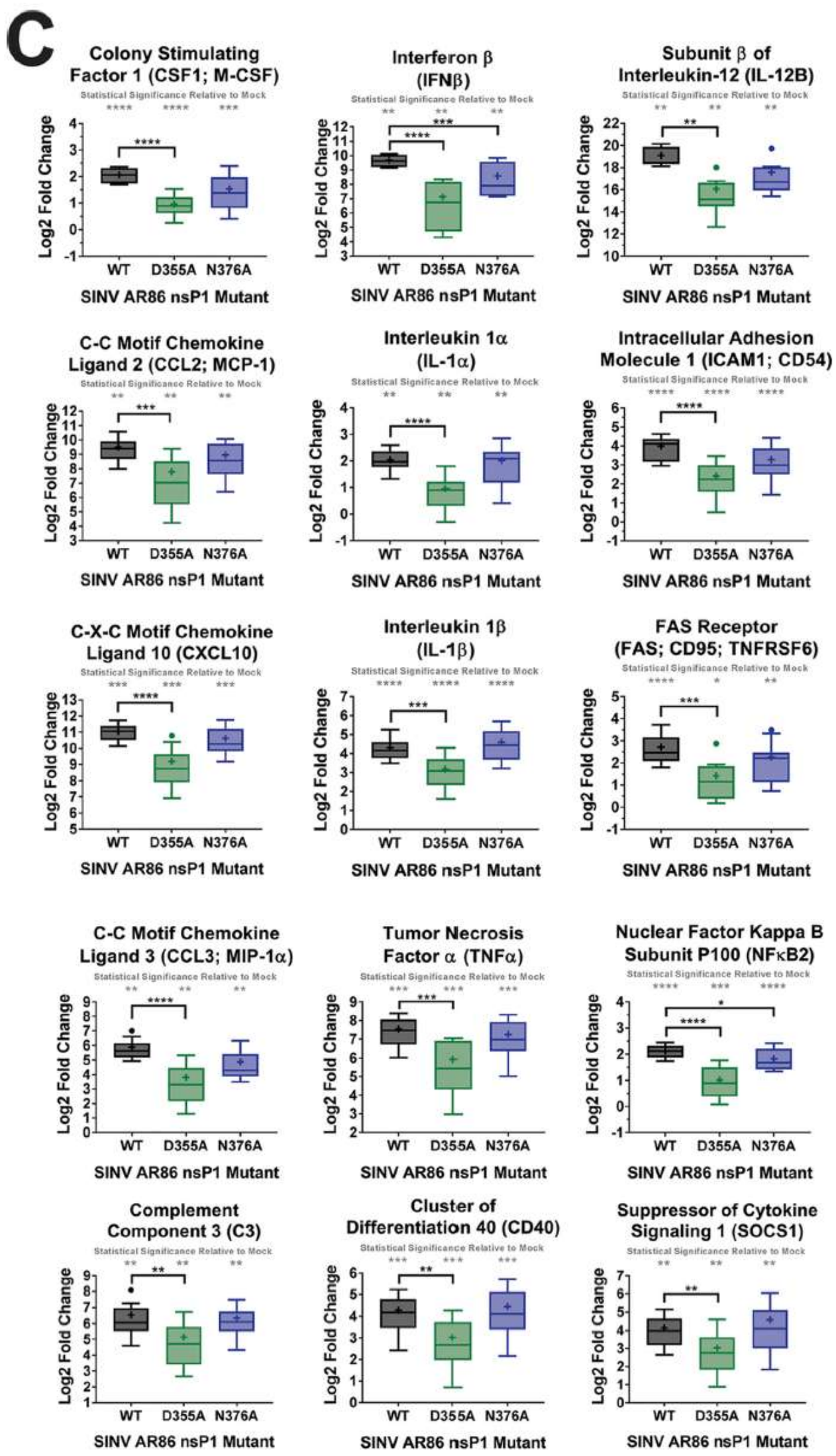
Figure 3.10. Increased viral capping efficiency results in reduced expression of pro-inflammatory genes in the brain. (A) Cytokine transcript levels in the brain at 7dpi were measured by qRT-PCR. Data was normalized to GAPDH and calculated relative to uninfected controls. (B) Volcano plot showing the fold change in transcript expression between wild type SINV and the D355A mutant. Green points are transcripts that have greater than a 2-fold change in expression and are significant according to the Benjamini and Hochberg corrected $p$ value. (C) Cytokines and chemokines whose expression was significantly increased compared to uninfected controls and exhibited a significant difference in expression between wild type SINV and the D355A mutant that was greater than 2 -fold. All the quantitative data shown represent means of results from at least three independent biological replicates, with center lines representing the median, + representing the mean, boxes representing the interquartile range, error bars representing standard deviation of the means, and - representing outliers as determined by Tukey's method. Statistical significance was determined by Student's $t$ test. 


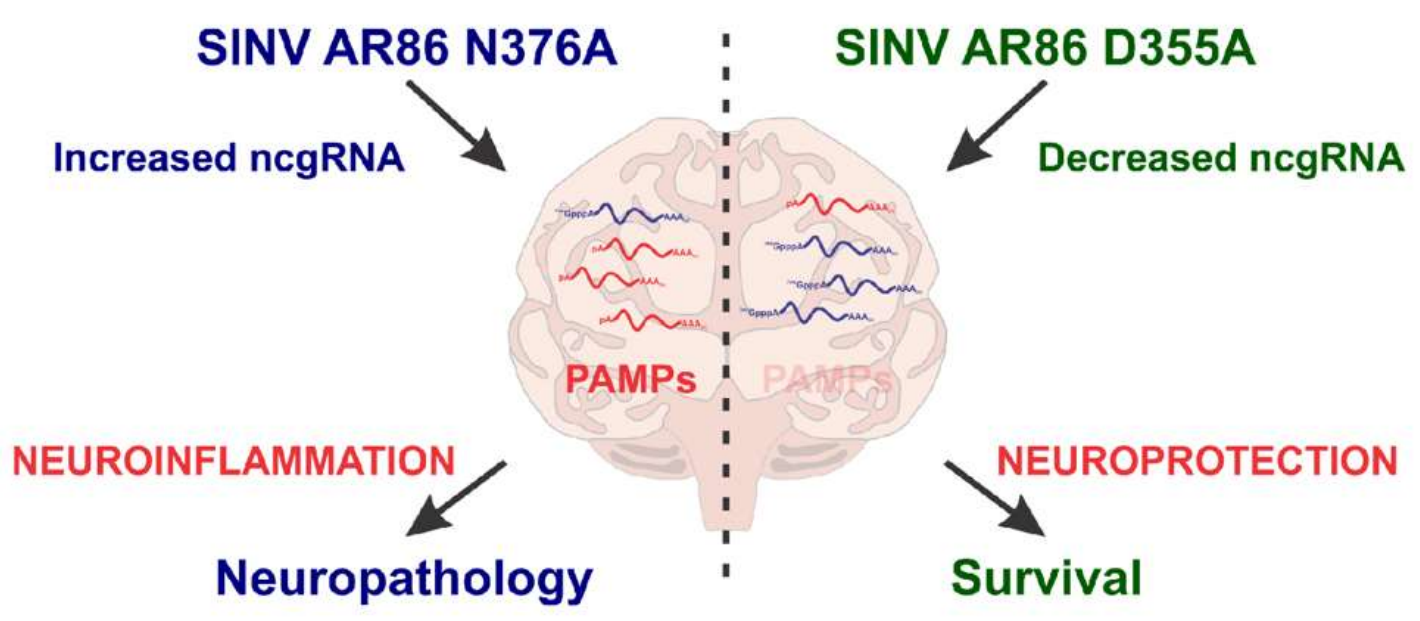

Figure 3.11. Proposed model of how increasing genomic vRNA capping negatively impacts viral pathogenesis. Infection of the brain with the decreased capping nsP1 mutant N376A results in increased production of ncgRNA, which likely act as PAMPs (left). The increased levels of PAMPs in the brain results in increased activation of the immune response, leading to excessive neuroinflammation and neuropathology. Conversely, infection of the brain with the increased capping nsP1 mutant D355A results in decreased production of ncgRNA, and therefore decreased production of PAMPs (right). The decreased levels of PAMPs results in a more moderate immune response that leads to neuroprotection and survival. 


\section{IFN $\beta$ Expression in Ankle Tissue at 1 Day Post Infection}

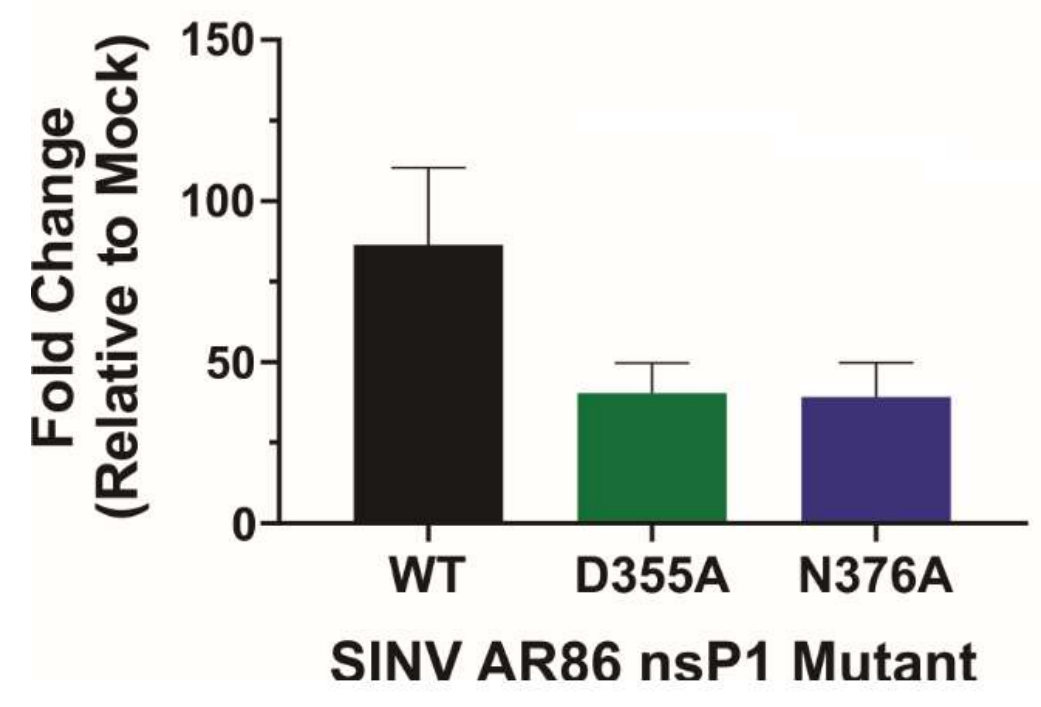

Supplemental Figure 3.1. Quantitative Analysis of IFN $\beta$ Transcript Levels in SINV AR86 Infected Ankle Tissue. Ankle tissues harvested from either mock infected, wild type SINV AR86, or SINV AR86 nsP1 mutant infected animals were harvested at 1 day post infection and homogenized. Total cellular RNA was isolated from the tissue homogenates and assessed via qRT-PCR to determine the relative transcript abundances of the IFN $\beta$ gene. Quantitative data shown are the means of at least three biological replicates, with the error bars representing the standard deviation of the means. Statistical analysis of the data, as by Student's T-Test, indicated no significant differences between the SINV AR86 infected tissues in regards to IFN $\beta$ transcript levels. 
Supplemental Table 3-1. Primers used in this study. Linker= F: GTTCAGAGTTCTACAGTCCGACCCATC

SINVg.CapAssay= R: CGTCTACGTTTACTACTGGCTTCTCC

SINV.nsP1 =F: AAGGATCTCCGGACCGTA,

R: AACATGAACTGGGTGGTGTCGAAG

SINV.E1= F: TCAGATGCACCACTGGTCTCAACA,

R: ATTGACCTTCGCGGTCGGATACAT

Mam.18S= F: CGCGGTTCTATTTTGTTGGT,

R: AGTCGGCATCGTTTATGGTC

IFIT2= F: AGTACAACGAGTAAGGAGTCACT,

R: AGGCCAGTATGTTGCACATGG

$\mathrm{SHB}=\mathrm{F}: \mathrm{CTGATGACTACTCCGATCCCTT}$,

R: GGGGTGTCGTACAACTGGATG

CXCL10= F: CCAAGTGCTGCCGTCATTTTC,

R: GGCTCGCAGGGATGATTTCAA

IFIH1= F: AGATCAACACCTGTGGTAACACC,

R: CTCTAGGGCCTCCACGAACA

Viperin= F: TGCTGGCTGAGAATAGCATTAGG,

R: GCTGAGTGCTGTTCCCATCT

MX2= F: GAGGCTCTTCAGAATGAGCAAA,

R: CTCTGCGGTCAGTCTCTCT

OAS2= F: TTGAAGAGGAATACATGCGGAAG,

R: GGGTCTGCATTACTGGCACTT 
BST2= F: TGTTCGGGGTTACCTTAGTCA,

R: GCAGGAGTTTGCCTGTGTCT

IFN $\beta=F:$ AAGAGTTACACTGCCTTTGCCATC,

R: CACTGTCTGCTGGTGGAGTTCATC

GAPDH= F: AGGTCGGTGTGAACGGATTTG,

R: TGTAGACCATGTAGTTGAGGTCA 


\section{CHAPTER 4 \\ DISCUSSION AND FUTURE DIRECTIONS}

\section{Research Summary}

Although the production and prevalence of the noncapped genomic RNA (ncgRNA) during alphaviral infection had been previously established, prior to these efforts, very little was known about their importance to viral infection and pathogenesis. By using mutations whose impacts on nsP1 enzymatic activity had been previously established in a similar virus outside of the context of infection using recombinant nsP1 protein, we were able to show that the capping activity of SINV nsP1 could be intentionally modulated during viral infection [124]. By altering the capping efficiency of nsP1 through the use of these point mutations, we were able to show that decreasing ncgRNA production negatively impacts viral infection. In characterizing these nsP1 capping mutants, we revealed that the decreased viral growth kinetics caused by decreasing ncgRNA production was not due to decreases in viral translation or vRNA synthesis, but rather was due to deficits in particle production. This marks the first time that the RNA capping activity of nsP1 has been directly implicated in determining particle production in any of the alphaviruses, outside of nsP1 mutations which altered production of viral proteins or vRNA synthesis. Furthermore, our results indicate 
that the ncgRNA specifically play an important role in regulating viral particle production.

In addition to the described impacts on viral infection in vitro, our data also establishes that the ncgRNA are vital to alphaviral pathogenesis, as decreasing ncgRNA production resulted in reduced morbidity and mortality as well as decreased inflammation, immune infiltration, and cell death in the brain.

Altogether, this was found to be due to the decreased expression of inflammatory cytokines in response to infection. While previous research with RRV had loosely correlated changes in ncgRNA production with disease progression, we were able to use the SINV nsP1 mutants to definitively show that the ncgRNA are major determinants of alphaviral pathogenesis [97, 99]. Furthermore, while ncgRNA had been previously found to be associated with activation of type-I IFN at the cellular level, we were able to expand upon this and show how altering ncgRNA production directly impacted activation of, as well as viral sensitivity to, type-I IFN. We also demonstrated that the ncgRNA were important for determining the host inflammatory response. Despite the wealth of evidentiary data connecting ncgRNAs to pathogenesis via the host response, exactly how the ncgRNA dictate disease severity and the host antiviral response is still unknown.

\section{Evolutionary Conservation of ncgRNA}

Multiple alphaviruses have been shown to produce ncgRNA during infection, indicating the ncgRNA production is conserved among the alphavirus 
genus. Since producing ncgRNA results in a greater immune response without lending an obvious advantage to viral replication in regards to viral gene expression or vRNA synthesis, there must be a separate reason for alphaviruses to have conserved their production as they are translationally inert and incapable of initiating infection by themselves. Our data suggests that alphaviruses may have conserved ncgRNA production in order to more efficiently replicate at the cellular level via the efficient production of viral particles. This is supported by the data in Chapter 2, where we showed that increasing capping impairs particle production in vitro. Nonetheless, in cellular models with an intact IFN response system, and in adult mouse models of infection, viral titer was similar between the D355A and wild type viruses (Chapter 3). It should be noted that in the IFNcompetent tissue culture model this was not due to increased infectious particle production in the D355A mutant, rather the production of wild type virus was reduced while D355A remained comparatively unaffected in regards to titer. We postulate that the equivalency between the wild type and D355A mutant in this scenario is due to the reduced induction of, and increased resistance to, type-I IFN by the D355A mutant which resulted in a slight advantage over the wild type virus despite decreased particle production. Similarly, although viral titer was not significantly different between D355A and wild type SINV in mice, this doesn't necessarily mean that the D355A did not still experience a defect in particle production in vivo, but rather could simply be explained as an effect of the D355A mutant being more resistant to IFN. If the ncgRNA play an important role in particle production in both in vitro and in vivo infections, then alphaviruses may 
have conserved ncgRNA production in order to more efficiently replicate at the cellular level. This would be especially important in the context of the reservoir host, where severe disease does not develop in response to wild type alphaviral infection. In this case, the increased particle production and higher viral titer that occurs when ncgRNA are present would be advantageous to the virus as it would allow for better viral transmission.

A second possibility is that alphaviruses produce ncgRNA because they are beneficial to viral transmission between the vertebrate host and invertebrate vectors, and vice versa. Previous work has shown that mosquitos fed alphaviral particles containing primarily capped genomic RNA have a significantly lower midgut infection rate compared to those that were fed primarily ncgRNA containing particles [123]. Furthermore, it has also been shown that, during vertebrate infection, the particles found in the serum are those associated with ncgRNA [123]. The fact that the particles containing mostly ncgRNA were also more infectious in mosquito models of infection strongly suggests that the ncgRNA are important for alphaviral transmission between the vertebrate host and the invertebrate vector. It is also interesting to note that infection of mosquitos using particles that contained primarily capped genomic RNA elicited a significantly weaker immune response in the midgut compared to the particles that contained mostly ncgRNA. The induction of an immune response in the midgut by the ncgRNA may function to help disrupt the midgut barrier, allowing for better dissemination of the virus throughout the mosquito. While more work would need to be done to characterize the importance of the ncgRNA to 
invertebrate infection as a whole, this does suggest that the ncgRNA play a critical role in establishing viral infection of the mosquito vector.

\section{Role of ncgRNA in Particle Production}

One particularly interesting finding that was described in Chapter 2 was that decreasing ncgRNA production alone had a significant detrimental impact to viral particle production. While it is unknown how the ncgRNA aid in viral particle production, there are multiple scenarios where the ncgRNAs could affect particle production.

One such scenario is that the ncgRNA bind a unique set of proteins that allow them to manipulate the cellular environment in favor of particle production (Fig. 4.1A). It stands to reason that if the capped genomic RNA are being translated in a manner similar to host mRNA, in that the RNA is lined with multiple ribosomes which all simultaneously translate the RNA, then the capped genomic RNA are not going to be able to efficiently interact with viral or host factors. In contrast, because the ncgRNA are not translated as they lack the canonical cap structure, translating polysomes would not form. This would allow the ncgRNAs to be available to interact with host/viral factors as well as maintain any secondary RNA structures that may be required for protein or RNA interaction [197]. Therefore, it is likely that the ncgRNA bind a different suite of proteins than the capped vRNA. While this has not been shown for alphaviral RNAs, the notion that non-translating RNAs bind different proteins than translating RNAs has been shown for eukaryotic mRNAs, especially in regards to 
P-body formation and translational silencing $[198,199]$. In relation to particle production, this means that the ncgRNA may either recruit proteins necessary for efficient particle production or sequester proteins which may inhibit particle assembly away from the vRNA population as a whole. This would allow the ncgRNA to mold the intracellular environment into one that is favorable for efficient particle production. Thus, by decreasing ncgRNA production with the D355A mutation, the virus's capacity to form a pro-assembly environment may have been reduced.

A second possibility is that the ncgRNA prevent premature disassembly of the nucleocapsid during the assembly process (Fig. 4.1B). Following entry of the viral particle into the host cell, the incoming nucleocapsid core is disassembled by the binding of the $60 \mathrm{~S}$ ribosomal subunit to the capsid protein [57]. Because the capped genomic RNA are associated with polysome formation and translation, there would be an abundance of $60 \mathrm{~S}$ ribosomal subunits bound to them or in close proximity. Once the virus begins assembling progeny particles, continued association of the 60S ribosomal subunit with the genomic RNA could potentially trigger premature disassembly of the nucleocapsid core before it can be adjoined to the viral glycoproteins and released as a mature particle. Therefore, it is likely that the nucleocapsid assembly microenvironment benefits from being devoid of ribosomal components and translating RNAs. Because the ncgRNA are not translated, they would not recruit the $60 \mathrm{~S}$ ribosomal subunit, and would not trigger premature disassembly of the viral capsid. Thus, it is possible that the ncgRNA may serve as a buffer between the areas where viral translation 
and particle assembly occur. However, as we know that both capped RNA and ncgRNA are packaged into viral particles and the packaging of capped vRNA is essential for viral infection, the ncgRNA buffer between the translating capped vRNA and the particle assembly area cannot by too restrictive, or no capped vRNA would be packaged into particles. The purpose of the ncgRNA buffer zone may then be to dilute the translating vRNA that are being packaged into particles, effectively reducing the amount of 605 ribosomal subunit present in a concentrated area, allowing for more efficient particle production.

Finally, the ncgRNA may act as allosteric effectors which aid in the formation of the nucleocapsid core (Fig. 4.1C). While this has not been explicitly shown for alphaviruses, the ability of viral RNA to allosterically regulate nucleocapsid assembly has been demonstrated in Rous sarcoma virus as well as bacteriophage MS2 [200]. In Rous sarcoma virus, particle assembly is inhibited by intramolecular interactions between different domains of the capsid protein Gag which prevent its dimerization. Binding of the viral RNA to Gag induces one or more conformational transitions that allows the protein to dimerize and assemble into a lattice structure [201]. In MS2, in addition to inducing capsid assembly, the viral RNA is also thought to act as a scaffold that directs the assembly of capsid proteins into the proper conformation for the viral particle [202]. Given that the specifics of the alphaviral capsid assembly are not currently known, it is possible that the ncgRNA may function as allosteric effectors that dictate the efficient assembly of nucleocapsid cores [66]. Therefore, decreasing 
ncgRNA production may have resulted in the decreased presence of an effector which may allow for efficient particle assembly.

\section{Role of ncgRNA in Pathogenesis}

A major finding of the in vivo characterization of the nsP1 mutants in Chapter 3 was that decreasing ncgRNA production resulted in little to no morbidity or mortality in mice. Interestingly, this was not due to defects in viral replication or tissue tropism, but rather this phenomenon was largely due to decreased expression of inflammatory molecules at the transcript level in the brain. This indicates that the ncgRNA are determinants of disease severity and that their presence plays a role in the activation / quality of the immune response. While exactly how the ncgRNA determine the host inflammatory response is unknown, it is likely to be at least in part due to the ncgRNA acting as PAMPs. Due to its lack of a 5' cap structure and likely increased secondary structure as they are not translated, the ncgRNA would serve as PAMPs that could be detected by multiple host innate immune proteins. As briefly stated in the introduction, RIG-I is responsible for detecting noncapped RNA, specifically RNAs with a 5' di- or triphosphate [203]. While the vast majority of the ncgRNA produced by SINV have a 5' monophosphate which is undetected by RIG-I, $\sim 20 \%$ of the ncgRNAs contain a $5^{\prime}$ di- or triphosphate, meaning that a small portion of the ncgRNA can potentially be detected by RIG-I [99]. In addition to being noncapped, the extensive branched secondary structures of ncgRNA may also serve as a PAMP for detection by innate immunity. Because translation of 
the RNA by ribosomes would result in the disruption of RNA secondary structure, it is likely that the non-translating ncgRNA would have significantly more secondary structure present than the capped genomic RNA, making the ncgRNA a target for dsRNA sensors like MDA5 and LGP2 as well.

The specific importance of MDA5 vs RIG-I in detecting alphaviral infection has not yet been firmly established; and in general seems to be dependent on viral strain, cell type, and mouse background [114, 204, 205]. The variation seen in the importance of RIG-I and MDA5 signaling to restricting viral infection may be in part due to the two proteins activating distinct response pathways that may be different or less prevalent depending on the context of infection. While there has been some evidence for CHIKV that RIG-I and MDA5 are redundant, work with flaviviruses has shown that they have unique roles in inducing expression of innate immune genes [206-208]. Therefore, detection of the ncgRNA by either one or both RIG-I and MDA5 may be responsible for the high levels of inflammation seen with wild type SINV infection and may account for why inflammation is reduced when ncgRNA production is decreased. However, more work is required to determine which of these immune sensors are specifically detecting the ncgRNA during alphaviral infection and to further establish that the role of the ncgRNA as PAMPS is directly tied to disease severity in vivo.

\section{ncgRNA as a Novel Antiviral Target}

Given the importance of the 5' cap to viral infection, it is unsurprising that the alphaviral capping enzyme has been a target for antiviral research. There 
have been multiple compounds developed to inhibit nsP1 capping activity. Some of these compounds directly interact with nsP1 to inhibit vRNA capping. For instance, MADTP-314 is known to directly disrupt the GTPase activity of nsP1, while FHA and FHNA were found to directly inhibit the MTase activity of nsP1 $[166,209]$. Although the exact mechanisms by which these compounds inhibit nsP1 is not known, they will likely become more clear in the near future now that the structure of nsP1 has been determined. Other compounds disrupt nsP1 capping activity in a more indirect manner. Many of these, such as ribavirin, are GTP analogs which can outcompete GTP for the nsP1 binding site and prevent the capping reaction from occurring $[168,210]$. Targeting SAM, which donates the methyl group to make the cap, is another route which prevents RNA capping that has been used for drug development [211]. Examples include Sinefungin, a SAM analog which has been shown to be able to inhibit transmethylation and disrupt nsP1 capping activity [212]. It is important to note that although many of these drugs have been shown to significantly reduce alphaviral replication in vitro, as of yet, none have been tested for efficacy against alphaviral infection in vivo.

The success of the nsP1 capping mutants to significantly impact morbidity and mortality in response to alphaviral infection highlights the ncgRNA as a novel target for antiviral development. While the antiviral studies mentioned above all focused on inhibiting nsP1 capping efficiency, which would result in increasing ncgRNA production, we have shown that SINV is significantly more sensitive to decreasing ncgRNA production than to equivalent increases in ncgRNA 
production. This was proven to be true for both viral replication at the molecular level as well as disease progression as a whole. The fact that SINV was less sensitive to decreases in capping activity is of particular interest in regards to the above mentioned antivirals, since if they do not sufficiently inhibit capping in vivo, they may not be efficacious. Furthermore, it is not known how increasing ncgRNA production, and therefore PAMP production, partway through infection may impact severity of disease, as treatment in the clinical setting often begins after the manifestation of pathology. Therefore, despite the counterintuitive approach of enhancing a viral replication process, decreasing ncgRNA production may be a more viable option for the development of viable antiviral therapies or preventatives than trying to reduce capped genomic RNA production to the point that it negatively impacts the virus.

\section{Future Directions}

While the work shown here establishes the importance of the ncgRNA to viral infection, there is still relatively little known about their specific functions during viral infection and the mechanism by which they influence pathogenesis. Therefore, future studies would endeavor to further characterize the ncgRNA and their roles during alphaviral infection. As stated above, this would include determining how ncgRNA influence particle production, identifying unique binding partners, characterizing how they are detected by innate immune sensors, and determining the potential of ncgRNA for the development of antivirals. Additionally, while ncgRNA production has been experimentally shown for SINV 
and RRV, characterizing ncgRNA production in other alphaviruses such as CHIKV or VEEV would help to establish their importance to alphaviral infection as a whole. Given the highly conserved nature of nsP1, it is likely that the inefficiency of 5' capping seen with SINV is true of the other alphaviruses as well, although the proportion of ncgRNA produced relative to capped genomic RNA will likely differ. In addition, future work which determines how alphaviruses evolve in response to mutations in nsP1 which alter capping efficiency, specifically whether there are direct reversions or compensatory mutations in the viral genome, may be able to shed further light on why ncgRNA production is conserved among the alphaviruses.

Also, although the work described here establishes the importance of the ncgRNA to alphaviral infection in mammals, how ncgRNA production affects infection of invertebrates has yet to be determined. It will be particularly interesting to see if altering ncgRNA production has a similar impact on viral infection and the antiviral response in whole mosquito models of infection. Since previous studies have shown that even altering the amount of ncgRNA present during the initial round of viral replication has drastic impacts on mosquito infection, maintaining these differences in ncgRNA production throughout the course of infection with the D355A capping mutant will potentially result in even greater deficits to viral infection.

Given the impact of altering ncgRNA production on disease severity, another future direction would include assessing the potential of the D355A mutant for vaccine development. While using the single point mutation by itself 
may not result in a viable vaccine due to the high likelihood of reversion, development of a virus with multiple mutations which affect capping may yield greater stability of the capping phenotype. A second approach would be to incorporate the D355A mutation into an already existing vaccine strain, such as VEEV TC83 and CHIKV 181/25, in order to reduce the high incidence of adverse effects while maintaining a high level of protection. 
A) ncgRNA Recruit/Sequester Host Proteins
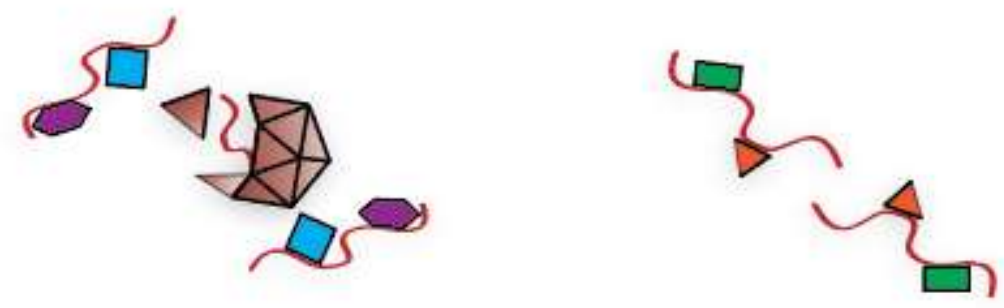

B) ncgRNA as a Buffer Between Translation and Particle Assembly

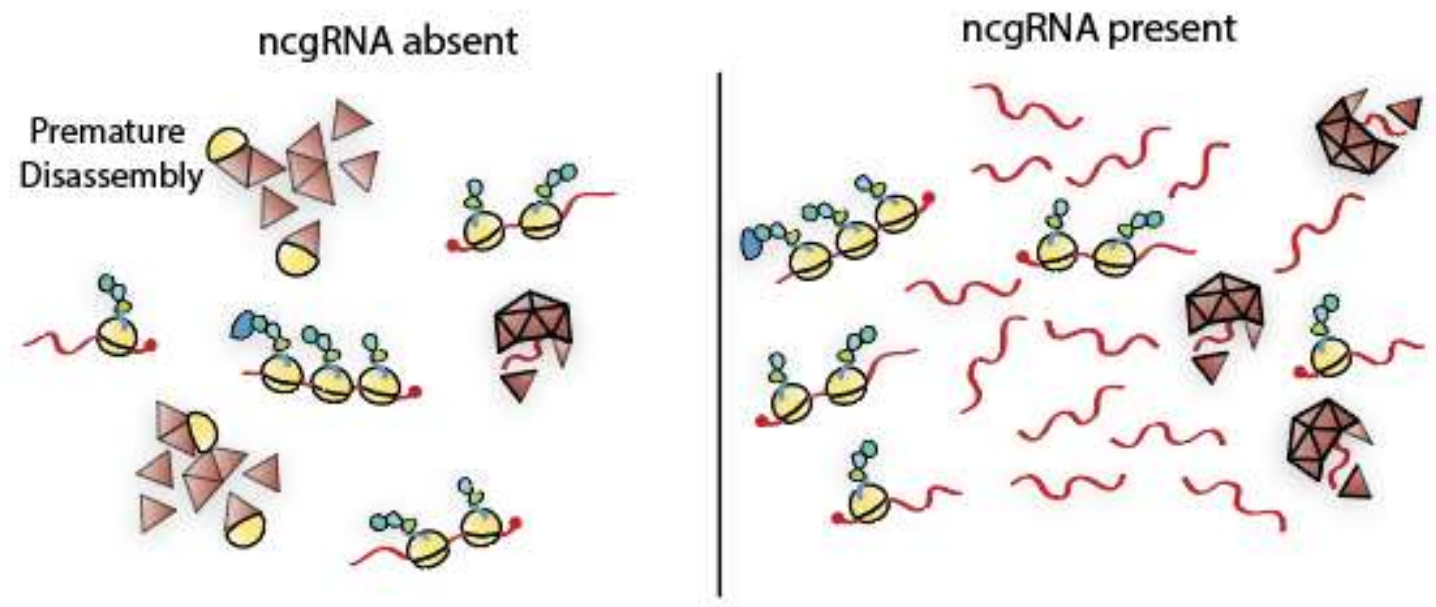

C) ncgRNA as Allosteric Effectors

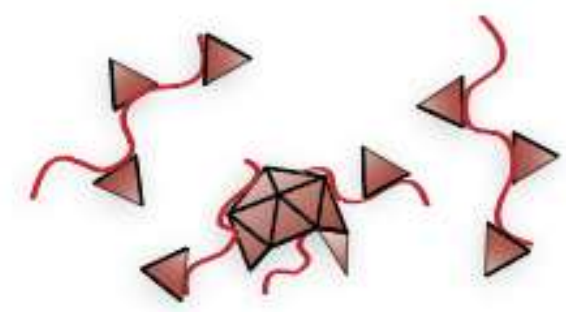

Figure 4.1. Potential roles for ncgRNA in alphaviral particle production. (A) Viral RNA (red lines) may bind different host or viral factors depending on whether or not the vRNA is capped. The ncgRNA may aid in particle assembly by recruiting proteins which make particle assembly more efficient (in purple and blue), or sequestering proteins which would impair particle assembly (in orange and green). (B) The 605 ribosomal subunit (large yellow half circles) are known to trigger particle disassembly. Therefore, having many translating capped vRNAs in the same area where particle assembly takes 
place may lead to increased association of the $60 \mathrm{~S}$ ribosomal subunit with viral capsid (red triangles) and lead to premature disassembly of the particles (Left). In this case, a potential function of the ncgRNA may be to serve as a buffer between the translating vRNAs and areas of particle assembly to prevent premature particle disassembly (Right). (C) Lastly, the ncgRNA may regulate particle assembly by acting as allosteric effectors. Binding of the capsid protein to the ncgRNA may allow for easier initiation of particle assembly. Additionally, the ncgRNA may act as a scaffold which direct proper assembly of the capsid proteins. 


\section{REFERENCES}

1. Griffin, D.E., Alphaviruses, in Fields Virology, D.M.K.a. P.M.Howley, Editor. 2001, Lippincott-Raven: Philadelphia, PA. p. 917-962.

2. Weaver, S.C., Urbanization and geographic expansion of zoonotic arboviral diseases: mechanisms and potential strategies for prevention. Trends Microbiol, 2013. 21(8): p. 360-3.

3. Kurkela, S., et al., Arthritis and arthralgia three years after Sindbis virus infection: clinical follow-up of a cohort of 49 patients. Scand J Infect Dis, 2008. 40(2): p. 167-73.

4. Sissoko, D., et al., Post-epidemic Chikungunya disease on Reunion Island: course of rheumatic manifestations and associated factors over a 15-month period. PLoS Negl Trop Dis, 2009. 3(3): p. e389.

5. Kurkela, S., et al., Clinical and laboratory manifestations of Sindbis virus infection: prospective study, Finland, 2002-2003. J Infect Dis, 2005. 191(11): p. 1820-9.

6. Kurkela, S., et al., Sindbis virus infection in resident birds, migratory birds, and humans, Finland. Emerg Infect Dis, 2008. 14(1): p. 41-7.

7. Farnon, E.C., J.J. Sejvar, and J.E. Staples, Severe disease manifestations associated with acute chikungunya virus infection. Crit Care Med, 2008. 36(9): p. 2682-3.

8. Fraser, J.R., Epidemic polyarthritis and Ross River virus disease. Clin Rheum Dis, 1986. 12(2): p. 369-88.

9. Cardona-Ospina, J.A., et al., Estimating the burden of disease and the economic cost attributable to chikungunya, Colombia, 2014. Trans R Soc Trop Med Hyg, 2015. 109(12): p. 793-802.

10. Seyler, T., et al., Estimating the burden of disease and the economic cost attributable to chikungunya, Andhra Pradesh, India, 2005-2006. Trans R Soc Trop Med Hyg, 2010. 104(2): p. 133-8.

11. Calisher, C.H., Medically important arboviruses of the United States and Canada. Clin Microbiol Rev, 1994. 7(1): p. 89-116.

12. de la Monte, S., et al., The systemic pathology of Venezuelan equine encephalitis virus infection in humans. Am J Trop Med Hyg, 1985. 34(1): p. 194-202.

13. Ronca, S.E., K.T. Dineley, and S. Paessler, Neurological Sequelae Resulting from Encephalitic Alphavirus Infection. Front Microbiol, 2016. 7: p. 959.

14. Griffin, D.E., Emergence and re-emergence of viral diseases of the central nervous system. Prog Neurobiol, 2010. 91(2): p. 95-101. 
15. Steele K., R.D., Glass P., Hart M., Ludwig G., Pratt W., Parker M., Smith J. , Chapter 12: Alphavirus Encephalitides. Medical Aspects of Biological Warfare, 2007: p. 241-270.

16. Franz, A.W., et al., Tissue Barriers to Arbovirus Infection in Mosquitoes. Viruses, 2015. 7(7): p. 3741-67.

17. Lim, E.X.Y., et al., Mosquitoes as Suitable Vectors for Alphaviruses. Viruses, 2018. 10(2).

18. Lee, W.S., et al., Mosquito antiviral defense mechanisms: a delicate balance between innate immunity and persistent viral infection. Parasit Vectors, 2019. 12(1): p. 165.

19. Cappuccio, L. and C. Maisse, Infection of Mammals and Mosquitoes by Alphaviruses: Involvement of Cell Death. Cells, 2020. 9(12).

20. Zacks, M.A. and S. Paessler, Encephalitic alphaviruses. Vet Microbiol, 2010. 140(3-4): p. 281-6.

21. Assuncao-Miranda, I., C. Cruz-Oliveira, and A.T. Da Poian, Molecular mechanisms involved in the pathogenesis of alphavirus-induced arthritis. Biomed Res Int, 2013. 2013: p. 973516.

22. DeTulleo, L. and T. Kirchhausen, The clathrin endocytic pathway in viral infection. EMBO J, 1998. 17(16): p. 4585-93.

23. Leung, J.Y., M.M. Ng, and J.J. Chu, Replication of alphaviruses: a review on the entry process of alphaviruses into cells. Adv Virol, 2011. 2011: p. 249640.

24. Helenius, A., et al., On the entry of Semliki forest virus into BHK-21 cells. J Cell Biol, 1980. 84(2): p. 404-20.

25. Omar, A. and H. Koblet, Semliki Forest virus particles containing only the E1 envelope glycoprotein are infectious and can induce cell-cell fusion. Virology, 1988. 166(1): p. 17-23.

26. Singh, I. and A. Helenius, Role of ribosomes in Semliki Forest virus nucleocapsid uncoating. J Virol, 1992. 66(12): p. 7049-58.

27. Shirako, Y. and J.H. Strauss, Regulation of Sindbis virus RNA replication: uncleaved $P 123$ and nsP4 function in minus-strand RNA synthesis, whereas cleaved products from $P 123$ are required for efficient plus-strand RNA synthesis. J Virol, 1994. 68(3): p. 1874-85.

28. Rupp, J.C., et al., Alphavirus RNA synthesis and non-structural protein functions. J Gen Virol, 2015. 96(9): p. 2483-500.

29. Melancon, P. and H. Garoff, Processing of the Semliki Forest virus structural polyprotein: role of the capsid protease. J Virol, 1987. 61(5): p. 1301-9.

30. Abdelnabi, R., J. Neyts, and L. Delang, Towards antivirals against chikungunya virus. Antiviral Res, 2015. 121: p. 59-68.

31. Kujala, P., et al., Biogenesis of the Semliki Forest virus RNA replication complex. J Virol, 2001. 75(8): p. 3873-84.

32. Spuul, P., et al., Role of the amphipathic peptide of Semliki forest virus replicase protein nsP1 in membrane association and virus replication. $\mathrm{J}$ Virol, 2007. 81(2): p. 872-83. 
33. Jones, R., et al., Capping pores of alphavirus nsP1 gate membranous viral replication factories. Nature, 2020.

34. Hur, S., Double-Stranded RNA Sensors and Modulators in Innate Immunity. Annu Rev Immunol, 2019. 37: p. 349-375.

35. Ahola, T., et al., Critical residues of Semliki Forest virus RNA capping enzyme involved in methyltransferase and guanylyltransferase-like activities. J Virol, 1997. 71(1): p. 392-7.

36. Laakkonen, P., et al., Expression of Semliki Forest virus nsP1-specific methyltransferase in insect cells and in Escherichia coli. J Virol, 1994. 68(11): p. 7418-25.

37. Laakkonen, P., T. Ahola, and L. Kaariainen, The effects of palmitoylation on membrane association of Semliki forest virus RNA capping enzyme. J Biol Chem, 1996. 271(45): p. 28567-71.

38. Ghosh, A. and C.D. Lima, Enzymology of RNA cap synthesis. Wiley Interdiscip Rev RNA, 2010. 1(1): p. 152-72.

39. Decroly, E., et al., Conventional and unconventional mechanisms for capping viral $m R N A$. Nat Rev Microbiol, 2011. 10(1): p. 51-65.

40. Vasiljeva, L., et al., Identification of a novel function of the alphavirus capping apparatus. RNA 5'-triphosphatase activity of Nsp2. J Biol Chem, 2000. 275(23): p. 17281-7.

41. Hardy, W.R. and J.H. Strauss, Processing the nonstructural polyproteins of sindbis virus: nonstructural proteinase is in the C-terminal half of nsP2 and functions both in cis and in trans. J Virol, 1989. 63(11): p. 4653-64.

42. Balistreri, G., et al., Enzymatic defects of the nsP2 proteins of Semliki Forest virus temperature-sensitive mutants. J Virol, 2007. 81(6): p. 284960.

43. Gorbalenya, A.E., et al., A novel superfamily of nucleoside triphosphatebinding motif containing proteins which are probably involved in duplex unwinding in DNA and RNA replication and recombination. FEBS Lett, 1988. 235(1-2): p. 16-24.

44. Russo, A.T., M.A. White, and S.J. Watowich, The crystal structure of the Venezuelan equine encephalitis alphavirus $n s P 2$ protease. Structure, 2006. 14(9): p. 1449-58.

45. Akhrymuk, I., S.V. Kulemzin, and E.I. Frolova, Evasion of the innate immune response: the Old World alphavirus $n s P 2$ protein induces rapid degradation of Rpb1, a catalytic subunit of RNA polymerase II. J Virol, 2012. 86(13): p. 7180-91.

46. Abraham, R., et al., ADP-ribosyl-binding and hydrolase activities of the alphavirus $n s P 3$ macrodomain are critical for initiation of virus replication. Proc Natl Acad Sci U S A, 2018. 115(44): p. E10457-E10466.

47. Jayabalan, A.K., et al., Stress granule formation, disassembly, and composition are regulated by alphavirus ADP-ribosylhydrolase activity. Proc Natl Acad Sci U S A, 2021. 118(6).

48. Gao, Y., et al., Multiple roles of the non-structural protein 3 (nsP3) alphavirus unique domain (AUD) during Chikungunya virus genome replication and transcription. PLoS Pathog, 2019. 15(1): p. e1007239. 
49. Kim, D.Y., et al., New World and Old World Alphaviruses Have Evolved to Exploit Different Components of Stress Granules, FXR and G3BP Proteins, for Assembly of Viral Replication Complexes. PLoS Pathog, 2016. 12(8): p. e1005810.

50. Li, G. and C.M. Rice, The signal for translational readthrough of a UGA codon in Sindbis virus RNA involves a single cytidine residue immediately downstream of the termination codon. J Virol, 1993. 67(8): p. 5062-7.

51. Rubach, J.K., et al., Characterization of purified Sindbis virus nsP4 RNAdependent RNA polymerase activity in vitro. Virology, 2009. 384(1): $p$. 201-8.

52. Coffey, L.L., et al., Arbovirus high fidelity variant loses fitness in mosquitoes and mice. Proc Natl Acad Sci U S A, 2011. 108(38): p. 1603843.

53. Carrasco, L., M.A. Sanz, and E. Gonzalez-Almela, The Regulation of Translation in Alphavirus-Infected Cells. Viruses, 2018. 10(2).

54. Krupovic, M. and E.V. Koonin, Multiple origins of viral capsid proteins from cellular ancestors. Proc Natl Acad Sci U S A, 2017. 114(12): p. E2401E2410.

55. Cheng, R.H., et al., Nucleocapsid and glycoprotein organization in an enveloped virus. Cell, 1995. 80(4): p. 621-30.

56. Lulla, V., et al., The amino-terminal domain of alphavirus capsid protein is dispensable for viral particle assembly but regulates RNA encapsidation through cooperative functions of its subdomains. J Virol, 2013. 87(22): $p$. 12003-19.

57. Wengler, G. and G. Wengler, In vitro analysis of factors involved in the disassembly of Sindbis virus cores by 60S ribosomal subunits identifies a possible role of low pH. J Gen Virol, 2002. 83(Pt 10): p. 2417-2426.

58. Hahn, C.S. and J.H. Strauss, Site-directed mutagenesis of the proposed catalytic amino acids of the Sindbis virus capsid protein autoprotease. J Virol, 1990. 64(6): p. 3069-73.

59. Choi, H.K., et al., Structure of Semliki Forest virus core protein. Proteins, 1997. 27(3): p. 345-59.

60. Bonatti, S., et al., Role of signal recognition particle in the membrane assembly of Sindbis viral glycoproteins. Eur J Biochem, 1984. 140(3): p. 499-502.

61. Liljestrom, P. and H. Garoff, Internally located cleavable signal sequences direct the formation of Semliki Forest virus membrane proteins from a polyprotein precursor. J Virol, 1991. 65(1): p. 147-54.

62. Zhang, X., et al., Furin processing and proteolytic activation of Semliki Forest virus. J Virol, 2003. 77(5): p. 2981-9.

63. Lobigs, M., J.M. Wahlberg, and H. Garoff, Spike protein oligomerization control of Semliki Forest virus fusion. J Virol, 1990. 64(10): p. 5214-8.

64. Weger-Lucarelli, J., et al., Identifying the Role of E2 Domains on Alphavirus Neutralization and Protective Immune Responses. PLoS Negl Trop Dis, 2015. 9(10): p. e0004163. 
65. Lescar, J., et al., The Fusion glycoprotein shell of Semliki Forest virus: an icosahedral assembly primed for fusogenic activation at endosomal $\mathrm{pH}$. Cell, 2001. 105(1): p. 137-48.

66. Mendes, A. and R.J. Kuhn, Alphavirus Nucleocapsid Packaging and Assembly. Viruses, 2018. 10(3).

67. Firth, A.E., et al., Discovery of frameshifting in Alphavirus $6 \mathrm{~K}$ resolves a 20-year enigma. Virol J, 2008. 5: p. 108.

68. Snyder, J.E., et al., Functional characterization of the alphavirus TF protein. J Virol, 2013. 87(15): p. 8511-23.

69. Gardner, J., et al., Chikungunya virus arthritis in adult wild-type mice. J Virol, 2010. 84(16): p. 8021-32.

70. Morrison, T.E., et al., A mouse model of chikungunya virus-induced musculoskeletal inflammatory disease: evidence of arthritis, tenosynovitis, myositis, and persistence. Am J Pathol, 2011. 178(1): p. 32-40.

71. Couderc, T., et al., A mouse model for Chikungunya: young age and inefficient type-l interferon signaling are risk factors for severe disease. PLoS Pathog, 2008. 4(2): p. e29.

72. Morrison, T.E., et al., Characterization of Ross River virus tropism and virus-induced inflammation in a mouse model of viral arthritis and myositis. J Virol, 2006. 80(2): p. 737-49.

73. Seymour, R.L., et al., A Rodent Model of Chikungunya Virus Infection in RAG1 -/- Mice, with Features of Persistence, for Vaccine Safety Evaluation. PLoS Negl Trop Dis, 2015. 9(6): p. e0003800.

74. Teo, T.H., et al., A pathogenic role for $C D 4+T$ cells during Chikungunya virus infection in mice. J Immunol, 2013. 190(1): p. 259-69.

75. Rulli, N.E., et al., Protection from arthritis and myositis in a mouse model of acute chikungunya virus disease by bindarit, an inhibitor of monocyte chemotactic protein-1 synthesis. J Infect Dis, 2011. 204(7): p. 1026-30.

76. Lum, F.M., et al., An essential role of antibodies in the control of Chikungunya virus infection. J Immunol, 2013. 190(12): p. 6295-302.

77. Pal, P., et al., Development of a highly protective combination monoclonal antibody therapy against Chikungunya virus. PLoS Pathog, 2013. 9(4): p. e1003312.

78. Griffin, D.E., Alphavirus Encephalomyelitis: Mechanisms and Approaches to Prevention of Neuronal Damage. Neurotherapeutics, 2016. 13(3): p. 455-60.

79. Thach, D.C., T. Kimura, and D.E. Griffin, Differences between C57BL/6 and $B A L B / c B y$ mice in mortality and virus replication after intranasal infection with neuroadapted Sindbis virus. J Virol, 2000. 74(13): p. 615661.

80. Reinarz, A.B., M.G. Broome, and B.P. Sagik, Age-dependent resistance of mice to sindbis virus infection: viral replication as a function of host age. Infect Immun, 1971. 3(2): p. 268-73.

81. Vernon, P.S. and D.E. Griffin, Characterization of an in vitro model of alphavirus infection of immature and mature neurons. J Virol, 2005. 79(6): p. 3438-47. 
82. Schultz, K.L., P.S. Vernon, and D.E. Griffin, Differentiation of neurons restricts Arbovirus replication and increases expression of the alpha isoform of IRF-7. J Virol, 2015. 89(1): p. 48-60.

83. Burdeinick-Kerr, R. and D.E. Griffin, Gamma interferon-dependent, noncytolytic clearance of sindbis virus infection from neurons in vitro. $\mathrm{J}$ Virol, 2005. 79(9): p. 5374-85.

84. Burdeinick-Kerr, R., J. Wind, and D.E. Griffin, Synergistic roles of antibody and interferon in noncytolytic clearance of Sindbis virus from different regions of the central nervous system. J Virol, 2007. 81(11): p. 5628-36.

85. Kerr, D.A., et al., BCL-2 and BAX protect adult mice from lethal Sindbis virus infection but do not protect spinal cord motor neurons or prevent paralysis. J Virol, 2002. 76(20): p. 10393-400.

86. Jan, J.T., S. Chatterjee, and D.E. Griffin, Sindbis virus entry into cells triggers apoptosis by activating sphingomyelinase, leading to the release of ceramide. J Virol, 2000. 74(14): p. 6425-32.

87. Nargi-Aizenman, J.L. and D.E. Griffin, Sindbis virus-induced neuronal death is both necrotic and apoptotic and is ameliorated by N-methyl-Daspartate receptor antagonists. J Virol, 2001. 75(15): p. 7114-21.

88. Kimura, T. and D.E. Griffin, Extensive immune-mediated hippocampal damage in mice surviving infection with neuroadapted Sindbis virus. Virology, 2003. 311(1): p. 28-39.

89. Jackson, A.C., et al., The pathogenesis of spinal cord involvement in the encephalomyelitis of mice caused by neuroadapted Sindbis virus infection. Lab Invest, 1987. 56(4): p. 418-23.

90. Kimura, T. and D.E. Griffin, The role of CD8(+) $T$ cells and major histocompatibility complex class I expression in the central nervous system of mice infected with neurovirulent Sindbis virus. J Virol, 2000. 74(13): p. 6117-25.

91. Rowell, J.F. and D.E. Griffin, Contribution of $T$ cells to mortality in neurovirulent Sindbis virus encephalomyelitis. J Neuroimmunol, 2002. 127(1-2): p. 106-14.

92. Binder, G.K. and D.E. Griffin, Interferon-gamma-mediated site-specific clearance of alphavirus from CNS neurons. Science, 2001. 293(5528): $p$. 303-6.

93. Levine, B., et al., Antibody-mediated clearance of alphavirus infection from neurons. Science, 1991. 254(5033): p. 856-60.

94. Levine, B. and D.E. Griffin, Persistence of viral RNA in mouse brains after recovery from acute alphavirus encephalitis. J Virol, 1992. 66(11): $p$. 6429-35.

95. Tyor, W.R., et al., Long term intraparenchymal Ig secretion after acute viral encephalitis in mice. J Immunol, 1992. 149(12): p. 4016-20.

96. Ahola, T., et al., Effects of palmitoylation of replicase protein nsP1 on alphavirus infection. J Virol, 2000. 74(15): p. 6725-33.

97. Stoermer Burrack, K.A., et al., Attenuating mutations in nsP1 reveal tissue-specific mechanisms for control of Ross River virus infection. J Virol, 2014. 88(7): p. 3719-32. 
98. Haist, K.C., et al., Inflammatory monocytes mediate control of acute alphavirus infection in mice. PLoS Pathog, 2017. 13(12): p. e1006748.

99. Sokoloski, K.J., et al., Noncapped Alphavirus Genomic RNAs and Their Role during Infection. J Virol, 2015. 89(11): p. 6080-92.

100. Heise, M.T., et al., An attenuating mutation in nsP1 of the Sindbis-group virus S.A.AR86 accelerates nonstructural protein processing and upregulates viral 26S RNA synthesis. J Virol, 2003. 77(2): p. 1149-56.

101. Simmons, J.D., A.C. Wollish, and M.T. Heise, A determinant of Sindbis virus neurovirulence enables efficient disruption of Jak/STAT signaling. J Virol, 2010. 84(21): p. 11429-39.

102. Heise, M.T., D.A. Simpson, and R.E. Johnston, $A$ single amino acid change in nsP1 attenuates neurovirulence of the Sindbis-group alphavirus S.A.AR86. J Virol, 2000. 74(9): p. 4207-13.

103. Fros, J.J., et al., Chikungunya virus nonstructural protein 2 inhibits type I/II interferon-stimulated JAK-STAT signaling. J Virol, 2010. 84(20): p. 1087787.

104. Simmons, J.D., et al., Venezuelan equine encephalitis virus disrupts STAT1 signaling by distinct mechanisms independent of host shutoff. $\mathrm{J}$ Virol, 2009. 83(20): p. 10571-81.

105. Bhalla, N., et al., Host translation shutoff mediated by non-structural protein 2 is a critical factor in the antiviral state resistance of Venezuelan equine encephalitis virus. Virology, 2016. 496: p. 147-165.

106. Dryga, S.A., O.A. Dryga, and S. Schlesinger, Identification of mutations in a Sindbis virus variant able to establish persistent infection in BHK cells: the importance of a mutation in the nsP2 gene. Virology, 1997. 228(1): $p$. 74-83.

107. McPherson, R.L., et al., ADP-ribosylhydrolase activity of Chikungunya virus macrodomain is critical for virus replication and virulence. Proc Natl Acad Sci U S A, 2017. 114(7): p. 1666-1671.

108. Atasheva, S., et al., Venezuelan equine encephalitis virus capsid protein inhibits nuclear import in Mammalian but not in mosquito cells. J Virol, 2008. 82(8): p. 4028-41.

109. Garmashova, N., et al., Analysis of Venezuelan equine encephalitis virus capsid protein function in the inhibition of cellular transcription. J Virol, 2007. 81(24): p. 13552-65.

110. Stanley, J., S.J. Cooper, and D.E. Griffin, Alphavirus neurovirulence: monoclonal antibodies discriminating wild-type from neuroadapted Sindbis virus. J Virol, 1985. 56(1): p. 110-9.

111. Kielian, $\mathrm{M}$. and A. Helenius, $\mathrm{pH}$-induced alterations in the fusogenic spike protein of Semliki Forest virus. J Cell Biol, 1985. 101(6): p. 2284-91.

112. Wahlberg, J.M., et al., Membrane fusion of Semliki Forest virus involves homotrimers of the fusion protein. J Virol, 1992. 66(12): p. 7309-18.

113. Tsetsarkin, K.A., et al., A single mutation in chikungunya virus affects vector specificity and epidemic potential. PLoS Pathog, 2007. 3(12): p. e201. 
114. Gardner, C.L., et al., Eastern and Venezuelan equine encephalitis viruses differ in their ability to infect dendritic cells and macrophages: impact of altered cell tropism on pathogenesis. J Virol, 2008. 82(21): p. 10634-46.

115. Hyde, J.L., et al., A viral RNA structural element alters host recognition of nonself RNA. Science, 2014. 343(6172): p. 783-7.

116. Kobiler, D., et al., A single nucleotide change in the 5 ' noncoding region of Sindbis virus confers neurovirulence in rats. J Virol, 1999. 73(12): p. 10440-6.

117. Kuhn, R.J., et al., Attenuation of Sindbis virus neurovirulence by using defined mutations in nontranslated regions of the genome RNA. J Virol, 1992. 66(12): p. 7121-7.

118. Hyde, J.L., et al., The 5' and $3^{\prime}$ ends of alphavirus RNAs--Non-coding is not non-functional. Virus Res, 2015. 206: p. 99-107.

119. Jones, J.E., et al., Disruption of the Opal Stop Codon Attenuates Chikungunya Virus-Induced Arthritis and Pathology. mBio, 2017. 8(6).

120. Tuittila, M.T., et al., Replicase complex genes of Semliki Forest virus confer lethal neurovirulence. J Virol, 2000. 74(10): p. 4579-89.

121. Myles, K.M., et al., Effects of an opal termination codon preceding the nsP4 gene sequence in the O'Nyong-Nyong virus genome on Anopheles gambiae infectivity. J Virol, 2006. 80(10): p. 4992-7.

122. Li, G.P. and C.M. Rice, Mutagenesis of the in-frame opal termination codon preceding nsP4 of Sindbis virus: studies of translational readthrough and its effect on virus replication. J Virol, 1989. 63(3): p. 1326-37.

123. Mackenzie-Liu, D., et al., Encapsidated Host Factors in Alphavirus Particles Influence Midgut Infection of Aedes aegypti. Viruses, 2018. 10(5).

124. Li, C., et al., mRNA Capping by Venezuelan Equine Encephalitis Virus nsP1: Functional Characterization and Implications for Antiviral Research. J Virol, 2015. 89(16): p. 8292-303.

125. LaPointe, A.T., et al., The Identification and Characterization of Sindbis Virus RNA:Host Protein Interactions. J Virol, 2018.

126. Adouchief, S., et al., Sindbis virus as a human pathogen-epidemiology, clinical picture and pathogenesis. Rev Med Virol, 2016. 26(4): p. 221-41.

127. Harley, D., A. Sleigh, and S. Ritchie, Ross River virus transmission, infection, and disease: a cross-disciplinary review. Clin Microbiol Rev, 2001. 14(4): p. 909-32, table of contents.

128. Rulli, N.E., et al., Ross River virus: molecular and cellular aspects of disease pathogenesis. Pharmacol Ther, 2005. 107(3): p. 329-42.

129. Pettersson, R.F., 5'-Terminal nucleotide sequence of Semliki forest virus $18 S$ defective interfering RNA is heterogeneous and different from the genomic 42S RNA. Proc Natl Acad Sci U S A, 1981. 78(1): p. 115-9.

130. Pettersson, R.F., H. Soderlund, and L. Kaariainen, The nucleotide sequences of the 5'-terminal T1 oligonucleotides of Semliki-Forest-virus 42-S and 26-S RNAs are different. Eur J Biochem, 1980. 105(3): p. 43543. 
131. Cancedda, R. and A.J. Shatkin, Ribosome-protected fragments from sindbis 42-S and 26-S RNAs. Eur J Biochem, 1979. 94(1): p. 41-50.

132. $\mathrm{Mi}, \mathrm{S}$. and V. Stollar, Expression of Sindbis virus nsP1 and methyltransferase activity in Escherichia coli. Virology, 1991. 184(1): $p$. 423-7.

133. Ahola, T. and L. Kaariainen, Reaction in alphavirus mRNA capping: formation of a covalent complex of nonstructural protein nsP1 with 7methyl-GMP. Proc Natl Acad Sci U S A, 1995. 92(2): p. 507-11.

134. Wang, H.L., J. O'Rear, and V. Stollar, Mutagenesis of the Sindbis virus nsP1 protein: effects on methyltransferase activity and viral infectivity. Virology, 1996. 217(2): p. 527-31.

135. Hefti, E., et al., 5' Nucleotide Sequence of Sindbis Viral RNA. J Virol, 1976. 17(1): p. 149-159.

136. Frolova, E., et al., Formation of nsP3-specific protein complexes during Sindbis virus replication. J Virol, 2006. 80(8): p. 4122-34.

137. Sokoloski, K.J., et al., Sindbis virus infectivity improves during the course of infection in both mammalian and mosquito cells. Virus Res, 2012. 167(1): p. 26-33.

138. Baer, A. and K. Kehn-Hall, Viral concentration determination through plaque assays: using traditional and novel overlay systems. J Vis Exp, 2014(93): p. e52065.

139. Stevens, A. and T.L. Poole, 5'-exonuclease-2 of Saccharomyces cerevisiae. Purification and features of ribonuclease activity with comparison to 5'-exonuclease-1. J Biol Chem, 1995. 270(27): p. 16063-9.

140. Jinek, M., S.M. Coyle, and J.A. Doudna, Coupled 5' nucleotide recognition and processivity in Xrn1-mediated mRNA decay. Mol Cell, 2011. 41(5): p. 600-8.

141. Garneau, N.L., et al., The 3' untranslated region of sindbis virus represses deadenylation of viral transcripts in mosquito and Mammalian cells. J Virol, 2008. 82(2): p. 880-92.

142. Song, M.G., S. Bail, and M. Kiledjian, Multiple Nudix family proteins possess mRNA decapping activity. RNA, 2013. 19(3): p. 390-9.

143. Hetzel, J., et al., Nascent RNA sequencing reveals distinct features in plant transcription. Proc Natl Acad Sci U S A, 2016. 113(43): p. 1231612321.

144. Best, M.D., Click chemistry and bioorthogonal reactions: unprecedented selectivity in the labeling of biological molecules. Biochemistry, 2009. 48(28): p. 6571-84.

145. Sokoloski, K.J., et al., Identification of Interactions between Sindbis Virus Capsid Protein and Cytoplasmic vRNA as Novel Virulence Determinants. PLoS Pathog, 2017. 13(6): p. e1006473.

146. Yang, J., et al., The I-TASSER Suite: protein structure and function prediction. Nat Methods, 2015. 12(1): p. 7-8.

147. Ahola, T. and D.G. Karlin, Sequence analysis reveals a conserved extension in the capping enzyme of the alphavirus supergroup, and a homologous domain in nodaviruses. Biol Direct, 2015. 10: p. 16. 
148. Shatkin, A.J., Capping of eucaryotic mRNAs. Cell, 1976. 9(4 PT 2): $p$. 645-53.

149. Banerjee, A.K., 5'-terminal cap structure in eucaryotic messenger ribonucleic acids. Microbiol Rev, 1980. 44(2): p. 175-205.

150. Sonenberg, N. and A.C. Gingras, The mRNA 5' cap-binding protein elF4E and control of cell growth. Curr Opin Cell Biol, 1998. 10(2): p. 268-75.

151. Fros, J.J. and G.P. Pijlman, Alphavirus Infection: Host Cell Shut-Off and Inhibition of Antiviral Responses. Viruses, 2016. 8(6).

152. Castello, A., et al., Translation of Sindbis virus 26S mRNA does not require intact eukariotic initiation factor 4G. J Mol Biol, 2006. 355(5): p. 942-56.

153. Kim, D.Y., et al., Conservation of a packaging signal and the viral genome RNA packaging mechanism in alphavirus evolution. J Virol, 2011. 85(16): p. 8022-36.

154. Rupp, J.C., N. Jundt, and R.W. Hardy, Requirement for the amino-terminal domain of sindbis virus nsP4 during virus infection. J Virol, 2011. 85(7): p. 3449-60.

155. Fata, C.L., S.G. Sawicki, and D.L. Sawicki, Modification of Asn374 of nsP1 suppresses a Sindbis virus nsP4 minus-strand polymerase mutant. J Virol, 2002. 76(17): p. 8641-9.

156. Shirako, Y., E.G. Strauss, and J.H. Strauss, Suppressor mutations that allow sindbis virus RNA polymerase to function with nonaromatic amino acids at the N-terminus: evidence for interaction between nsP1 and nsP4 in minus-strand RNA synthesis. Virology, 2000. 276(1): p. 148-60.

157. Kumar, S., et al., Chikungunya virus nsP1 interacts directly with nsP2 and modulates its ATPase activity. Sci Rep, 2018. 8(1): p. 1045.

158. Jupille, H.J., et al., Mutations in nsP1 and PE2 are critical determinants of Ross River virus-induced musculoskeletal inflammatory disease in a mouse model. Virology, 2011. 410(1): p. 216-27.

159. Kallio, K., et al., RNA Replication and Membrane Modification Require the Same Functions of Alphavirus Nonstructural Proteins. J Virol, 2016. 90(3): p. 1687-92.

160. Gonzalez-Almela, E., et al., Differential action of pateamine A on translation of genomic and subgenomic mRNAs from Sindbis virus. Virology, 2015. 484: p. 41-50.

161. Patel, R.K., et al., Role for subgenomic mRNA in host translation inhibition during Sindbis virus infection of mammalian cells. Virology, 2013. 441(2): p. 171-81.

162. Wengler, G. and G. Wengler, Identification of a transfer of viral core protein to cellular ribosomes during the early stages of alphavirus infection. Virology, 1984. 134(2): p. 435-42.

163. LaPointe, A.T., et al., Production of Noncapped Genomic RNAs Is Critical to Sindbis Virus Disease and Pathogenicity. mBio, 2020. 11(6).

164. Schwartz, O. and M.L. Albert, Biology and pathogenesis of chikungunya virus. Nat Rev Microbiol, 2010. 8(7): p. 491-500. 
165. Simpson, D.A., et al., Complete nucleotide sequence and full-length cDNA clone of S.A.AR86 a South African alphavirus related to Sindbis. Virology, 1996. 222(2): p. 464-9.

166. Kovacikova, K., et al., 6'-beta-Fluoro-Homoaristeromycin and 6'-FluoroHomoneplanocin A Are Potent Inhibitors of Chikungunya Virus Replication through Their Direct Effect on Viral Nonstructural Protein 1. Antimicrob Agents Chemother, 2020. 64(4).

167. Feibelman, K.M., et al., Identification of small molecule inhibitors of the Chikungunya virus nsP1 RNA capping enzyme. Antiviral Res, 2018. 154: p. 124-131.

168. Bullard-Feibelman, K.M., B.P. Fuller, and B.J. Geiss, A Sensitive and Robust High-Throughput Screening Assay for Inhibitors of the Chikungunya Virus nsP1 Capping Enzyme. PLoS One, 2016. 11(7): p. e0158923.

169. Lampio, A., et al., Guanosine nucleotide analogs as inhibitors of alphavirus mRNA capping enzyme. Antiviral Res, 1999. 42(1): p. 35-46.

170. Wollish, A.C., et al., An attenuating mutation in a neurovirulent Sindbis virus strain interacts with the IPS-1 signaling pathway in vivo. Virology, 2013. 435(2): p. 269-80.

171. LaPointe, A.T., J. Moreno-Contreras, and K.J. Sokoloski, Increasing the Capping Efficiency of the Sindbis Virus nsP1 Protein Negatively Affects Viral Infection. Mbio, 2018. 9(6).

172. Chomczynski, P. and M. Rymaszewski, Alkaline polyethylene glycolbased method for direct PCR from bacteria, eukaryotic tissue samples, and whole blood. Biotechniques, 2006. 40(4): p. 454, 456, 458.

173. Ribble, D., et al., A simple technique for quantifying apoptosis in 96-well plates. BMC Biotechnol, 2005. 5: p. 12.

174. Garmashova, N., et al., The Old World and New World alphaviruses use different virus-specific proteins for induction of transcriptional shutoff. J Virol, 2007. 81(5): p. 2472-84.

175. Frolov, I., et al., Early events in alphavirus replication determine the outcome of infection. J Virol, 2012. 86(9): p. 5055-66.

176. Pervolaraki, K., et al., Differential induction of interferon stimulated genes between type I and type III interferons is independent of interferon receptor abundance. PLoS Pathog, 2018. 14(11): p. e1007420.

177. Johnson, R.T., H.F. McFarland, and S.E. Levy, Age-dependent resistance to viral encephalitis: studies of infections due to Sindbis virus in mice. J Infect Dis, 1972. 125(3): p. 257-62.

178. Griffin, D.E., Role of the immune response in age-dependent resistance of mice to encephalitis due to Sindbis virus. J Infect Dis, 1976. 133(4): p. $456-64$.

179. Lewis, J., et al., Alphavirus-induced apoptosis in mouse brains correlates with neurovirulence. J Virol, 1996. 70(3): p. 1828-35.

180. Levine, B., et al., Bc1-2 protects mice against fatal alphavirus encephalitis. Proc Natl Acad Sci U S A, 1996. 93(10): p. 4810-5. 
181. Jackson, A.C., et al., Basis of neurovirulence in Sindbis virus encephalomyelitis of mice. Lab Invest, 1988. 58(5): p. 503-9.

182. Buschmann, J.P., et al., Inflammatory response and chemokine expression in the white matter corpus callosum and gray matter cortex region during cuprizone-induced demyelination. J Mol Neurosci, 2012. 48(1): p. 66-76.

183. Huang da, W., B.T. Sherman, and R.A. Lempicki, Systematic and integrative analysis of large gene lists using DAVID bioinformatics resources. Nat Protoc, 2009. 4(1): p. 44-57.

184. Huang da, W., B.T. Sherman, and R.A. Lempicki, Bioinformatics enrichment tools: paths toward the comprehensive functional analysis of large gene lists. Nucleic Acids Res, 2009. 37(1): p. 1-13.

185. Baxter, V.K. and D.E. Griffin, Interferon gamma modulation of disease manifestation and the local antibody response to alphavirus encephalomyelitis. J Gen Virol, 2016. 97(11): p. 2908-2925.

186. Yin, J., et al., Similarities and differences in antagonism of neuron alpha/beta interferon responses by Venezuelan equine encephalitis and Sindbis alphaviruses. J Virol, 2009. 83(19): p. 10036-47.

187. Gorchakov, R., E. Frolova, and I. Frolov, Inhibition of transcription and translation in Sindbis virus-infected cells. J Virol, 2005. 79(15): p. 9397409.

188. Frolova, E.I., et al., Roles of nonstructural protein nsP2 and Alpha/Beta interferons in determining the outcome of Sindbis virus infection. J Virol, 2002. 76(22): p. 11254-64.

189. Liu, X., et al., Decreased Virulence of Ross River Virus Harboring a Mutation in the First Cleavage Site of Nonstructural Polyprotein Is Caused by a Novel Mechanism Leading to Increased Production of InterferonInducing RNAs. mBio, 2018. 9(4).

190. Nikonov, A., et al., RIG-I and MDA-5 detection of viral RNA-dependent RNA polymerase activity restricts positive-strand RNA virus replication. PLoS Pathog, 2013. 9(9): p. e1003610.

191. Kawai, T. and S. Akira, Antiviral signaling through pattern recognition receptors. J Biochem, 2007. 141(2): p. 137-45.

192. Havert, M.B., et al., Activation of divergent neuronal cell death pathways in different target cell populations during neuroadapted sindbis virus infection of mice. J Virol, 2000. 74(11): p. 5352-6.

193. Greene, I.P., et al., Protection from fatal viral encephalomyelitis: AMPA receptor antagonists have a direct effect on the inflammatory response to infection. Proc Natl Acad Sci U S A, 2008. 105(9): p. 3575-80.

194. Kulcsar, K.A., et al., Interleukin 10 modulation of pathogenic Th17 cells during fatal alphavirus encephalomyelitis. Proc Natl Acad Sci U S A, 2014. 111(45): p. 16053-8.

195. Cruz, C.C., et al., Modulation of type I IFN induction by a virulence determinant within the alphavirus nsP1 protein. Virology, 2010. 399(1): $p$. $1-10$. 
196. Akhrymuk, I., I. Frolov, and E.I. Frolova, Both RIG-I and MDA5 detect alphavirus replication in concentration-dependent mode. Virology, 2016. 487: p. 230-41.

197. Khong, A. and R. Parker, The landscape of eukaryotic mRNPs. RNA, 2020. 26(3): p. 229-239.

198. Mazan-Mamczarz, K., et al., Translational repression by RNA-binding protein TIAR. Mol Cell Biol, 2006. 26(7): p. 2716-27.

199. Teixeira, D., et al., Processing bodies require RNA for assembly and contain nontranslating mRNAs. RNA, 2005. 11(4): p. 371-82.

200. Zlotnick, A. and S. Mukhopadhyay, Virus assembly, allostery and antivirals. Trends Microbiol, 2011. 19(1): p. 14-23.

201. Taylor, G.M., et al., NMR relaxation studies of an RNA-binding segment of the rous sarcoma virus gag polyprotein in free and bound states: a model for autoinhibition of assembly. Biochemistry, 2010. 49(19): p. 4006-17.

202. Basnak, G., et al., Viral genomic single-stranded RNA directs the pathway toward a T=3 capsid. J Mol Biol, 2010. 395(5): p. 924-36.

203. Ren, X., et al., RIG-I Selectively Discriminates against 5'-Monophosphate RNA. Cell Rep, 2019. 26(8): p. 2019-2027 e4.

204. Aguilar, P.V., et al., Variation in interferon sensitivity and induction among strains of eastern equine encephalitis virus. J Virol, 2005. 79(17): p. 11300-10.

205. White, L.J., et al., Role of alpha/beta interferon in Venezuelan equine encephalitis virus pathogenesis: effect of an attenuating mutation in the $5^{\prime}$ untranslated region. J Virol, 2001. 75(8): p. 3706-18.

206. Schilte, C., et al., Type I IFN controls chikungunya virus via its action on nonhematopoietic cells. J Exp Med, 2010. 207(2): p. 429-42.

207. Errett, J.S., et al., The essential, nonredundant roles of RIG-I and MDA5 in detecting and controlling West Nile virus infection. J Virol, 2013. 87(21): $p$. 11416-25.

208. Loo, Y.M., et al., Distinct RIG-I and MDA5 signaling by RNA viruses in innate immunity. J Virol, 2008. 82(1): p. 335-45.

209. Delang, L., et al., The viral capping enzyme nsP1: a novel target for the inhibition of chikungunya virus infection. Sci Rep, 2016. 6: p. 31819.

210. Rabah, N., et al., Mutations on VEEV nsP1 relate RNA capping efficiency to ribavirin susceptibility. Antiviral Res, 2020. 182: p. 104883.

211. Zhang, J. and Y.G. Zheng, SAM/SAH Analogs as Versatile Tools for SAMDependent Methyltransferases. ACS Chem Biol, 2016. 11(3): p. 583-97.

212. Kaur, R., et al., Development of an ELISA assay for screening inhibitors against divalent metal ion dependent alphavirus capping enzyme. Virus Res, 2018. 256: p. 209-218. 


\section{APPENDIX}

\section{Abbreviation}

\begin{tabular}{|c|c|}
\hline ANOVA & Analysis of Variance \\
\hline AUD & Alphavirus Unique Domain \\
\hline Bax & Bcl-2 Associated $X$ protein \\
\hline $\mathrm{Bcl}-2$ & B-cell lymphoma 2 \\
\hline $\mathrm{BHK}$ & Baby hamster kidney cells \\
\hline BST-2 & Bone Marrow Stromal Cell Antigen 2 \\
\hline CCL2 & C-C Motif Chemokine Ligand 2 \\
\hline CCL3 & C-C Motif Chemokine Ligand 3 \\
\hline CHIKV & Chikungunya virus \\
\hline CNS & Central nervous system \\
\hline CXCL10 & C-X-C Motif Chemokine Ligand 10 \\
\hline DAMP & Damage-associated molecular pattern \\
\hline dpi & Days post infection \\
\hline dsRNA & Double-stranded RNA \\
\hline $\mathrm{EB} / \mathrm{AO}$ & Ethidium bromide/ acridine orange \\
\hline EEEV & Eastern Equine Encephalitis virus \\
\hline ER & Endoplasmic reticulum \\
\hline FBS & Fetal Bovine Serum \\
\hline
\end{tabular}




\begin{tabular}{|c|c|}
\hline GAPDH & Glyceraldehyde 3-phosphate dehydrogenase \\
\hline GFP & Green Fluorescent Protein \\
\hline$H \& E$ & Haematoxylin and eosin \\
\hline hpi & Hours post infection \\
\hline $\mathrm{IFIH} 1$ & Interferon Induced With Helicase C Domain 1 \\
\hline IFIT1 & Interferon Induced Protein With Tetratricopeptide \\
\hline & Repeats 1 \\
\hline IFN & Interferon \\
\hline IFNAR & Interferon alpha/beta receptor \\
\hline IL-1 & Interleukin-1 \\
\hline IL-10 & Interleukin-10 \\
\hline IRF & Interferon response factor \\
\hline ISG & Interferon stimulated gene \\
\hline IU & Infectious unit, International unit \\
\hline JAK & Janus kinase \\
\hline LGP2 & Laboratory of Genetics and Physiology 2 \\
\hline MAVS & Mitochondrial antiviral-signaling protein \\
\hline MCP-1 & Monocyte chemoattractant protein 1 \\
\hline MDA5 & Melanoma differentiation-associated protein 5 \\
\hline MEM & Minimal Essential Media \\
\hline $\mathrm{MOI}$ & Multiplicity of Infection \\
\hline mRNA & Messenger RNA \\
\hline $\mathrm{M} \times 2$ & Myxovirus Resistance Protein 2 \\
\hline
\end{tabular}




\begin{tabular}{|c|c|}
\hline ncgRNA & Noncapped genomic RNA \\
\hline nsP & Nonstructural protein \\
\hline OAS2 & 2'-5'-Oligoadenylate Synthetase 2 \\
\hline ONNV & O'nyong'nyong virus \\
\hline PAMP & Pathogen-associated molecular pattern \\
\hline PBS & Phosphate Buffered Saline \\
\hline PFU & Plaque Forming Units \\
\hline PNK & Polynucleotide Kinase \\
\hline qPCR & Quantitative Polymerase Chain Reaction \\
\hline qRT-PCR & Quantitative Real Time Polymerase Chain Reaction \\
\hline $\operatorname{RdRp}$ & RNA dependent RNA polymerase \\
\hline RIG-I & Retinoic acid-inducible gene-I \\
\hline $\mathrm{RppH}$ & RNA 5' Pyrophosphohydrolase \\
\hline RRV & Ross River virus \\
\hline RT & Reverse Transcription \\
\hline SAM & S-adenosylmethionine \\
\hline SFV & Semliki Forest virus \\
\hline SINV & Sindbis virus \\
\hline socs & Suppressor of Cytokine Signaling \\
\hline ssRNA & Single-stranded RNA \\
\hline STAT & Signal transducer and activator of transcription protein \\
\hline TF & Transframe protein \\
\hline TGFb & Transforming Growth Factor beta \\
\hline
\end{tabular}


TNFa

Tyk2

VEEV

vRNA

WEEV

WT

$\mathrm{XRN}-1$
Tumor Necrosis Factor alpha

Tyrosine Kinase 2

Venezuelan Equine Encephalitis virus

viral RNA

Western Equine Encephalitis virus

Wild type

5'-3' exoribonuclease 1 


\section{CURRICULUM VITAE}

Autumn Towne LaPointe

University of Louisville

Clinical Translational Research Building Rm 642 E

Louisville, KY 40292

Altown02@louisville.edu

\section{EDUCATION}

05/2018 M.S. in Microbiology and Immunology, University of Louisville, Louisville, KY.

05/2016 B.S. in Cellular and Molecular Biology, Minor in Mathematics, University of North Carolina Asheville (UNCA), Asheville, NC

\section{RESEARCH EXPERIENCE}

08/2016-Current Graduate Research Assistant, Advisor: Dr. Kevin Sokoloski.

Developed vRNA capping mutants for Sindbis virus and determined the impact of noncapped genomic vRNA on alphaviral infection and pathogenesis.

11/2014-05/2016 Undergraduate Research in Biology, Advisor: Dr. Thomas Meigs. Characterized the protein:protein interaction site between Ga12 and AKAP-LBC.

01/2014-05/2015 Undergraduate Research in Mathematics, Advisor: Dr. Samuel Kaplan.

Explored the impact of low math self-efficacy on mathematical ability.

\section{TEACHING EXPERIENCE}

\section{UNIVERSITY OF LOUISVILLE}

$06 / 2019-07 / 2019$

$01 / 2019-08 / 2019$

$2018,2019,2020$
Completed UofL Mentor Academy Mentored Undergraduate Research Project in Virology Taught In Vitro Virology Methods in Methods Course 


\section{UNIVERSITY OF NORTH CAROLINA ASHEVILLE}

08/2014-05/2016 Embedded Tutor for Ecology and Evolution, Cellular and Molecular Biology, and Botany Courses

NORTH CAROLINA STATE UNIVERSITY \& DUKE UNIVERSITY

06/2015-07/2015 Summer Institute for Training in Biostatistics

\section{PROFESSIONAL MEMBERSHIPS AND ACTIVITIES}

2018-CurrentAmerican Society for Virology (Student Member) 2018-CurrentAmerican Society for Microbiology (Student Member) 2017-Current Science Policy and Outreach Group, UofL 2016-CurrentMicrobiology and Immunology Student Organization, UofL 2013-2016 Phi Eta Sigma Honor Society Member, UNCA

\section{HONORS AND AWARDS}

2020 American Society for Virology, Student Travel Award, 39 th

2020 Best Oral Presentation Award, Viruses 2020- Novel

2019 Research!Louisville $1^{\text {st }}$ place Doctoral Basic-Science Graduate Student Award

2019 Graduate Student Council Travel Award, UofL

2019 American Society for Virology, Student Travel Award, 38 ${ }^{\text {th }}$ Annual Meeting

2018-2020 T32 Grant Recipient

2018 American Society for Virology, Student Travel Award, $37^{\text {th }}$ Annual Meeting

2016 Harry H. Johnston Award for Excellence in Biology, UNCA

2016 Manly E. Wright Valedictorian Award Nominee, UNCA

2016

2012-2016

2012-2016 Graduated summa cum laude, UNCA Honor's Program, UNCA Laurel's Scholar; merit scholarship, UNCA

\section{ABSTRACTS AND PRESENTATIONS}

\section{ORAL PRESENTATIONS}


12/2020 "Production of Non-capped Genomic RNAs is Critical to Sindbis Virus Pathology." American Society for Microbiology Kentucky-Tennessee Regional Meeting, Virtual Presentation.

06/2020 "Alphaviral Pathogenesis is Dependent on the Production of Noncapped Viral RNA." $39^{\text {th }}$ Annual Meeting of the American Society for Virology, Virtual Presentation.

02/2020 "Noncapped Genomic RNAs are Critical for Alphaviral Infection and Pathogenicity." Novel Concepts in Virology Viruses Conference, Barcelona, Spain.

10/2019 "Increasing the Production of Capped Alphaviral RNAs Negatively Impacts Viral Pathogenicity". Midwest Virology Symposium, Cleveland, $\mathrm{OH}$.

02/2019 "Increasing the Capping Efficiency of the Sindbis Virus nsP1 Protein Negatively Affects Viral Infection." Graduate Student Regional Research Conference. Louisville, KY.

07/2018 "Identifying the Role of Noncapped Alphaviral RNAs During Infection". $37^{\text {th }}$ Annual Meeting of the American Society for Virology, College Park, MD

04/2016 "Determinants of Ga12-specific binding to AKAP-Lbc". National Conference of Undergraduate Research Annual Meeting, Asheville, NC

\section{POSTER PRESENTATIONS}

10/2019 "Increasing the Production of Capped Alphaviral RNAs Negatively Impacts Viral Pathogenicity". Midwest Virology Symposium, Cleveland, $\mathrm{OH}$.

09/2019 "Increasing the Production of Capped Alphaviral RNAs Negatively Impacts Viral Pathogenicity". Research!Louisville, Louisville, KY.

07/2019 "Increasing the Capping Efficiency of the Sindbis Virus nsP1 Protein Negatively Affects Viral Infection and Alters Viral Pathogenesis". $38^{\text {th }}$ Annual Meeting of the American Society for Virology, Minneapolis, MN

05/2019 "Increasing the Capping Efficiency of the Sindbis Virus nsP1 Protein Negatively Affects Viral Infection and Alters Viral 
Pathogenesis". Viruses and Cells Gordon Research Conference, Barga, Italy.

05/2019 "Increasing the Capping Efficiency of the Sindbis Virus nsP1 Protein Negatively Affects Viral Infection and Alters Viral Pathogenesis". Viruses and Cells Gordan Research Seminar, Barga, Italy.

10/2018 "Identifying the Role of Noncapped Alphaviral RNAs During Infection". American Society for Microbiology KentuckyTennessee Regional Meeting, Murfreesboro, TN

09/2018 "Identifying the Role of Noncapped Alphaviral RNAs During Infection". Research!Louisville, Louisville, KY

09/2017 "Noncapped but NOT Nonfunctional- Identifying the Role of Noncapped Alphaviral RNAs During Infection". Research!Louisville, Louisville, KY.

08/2017 "Noncapped but NOT Nonfunctional- Identifying the Role of Noncapped Alphaviral RNAs During Infection". 24 $4^{\text {th }}$ Midwest Microbial Pathogenesis Conference, Notre Dame, IN

04/2016 "Determinants of Ga12-specific binding common to AKAPLbc and p114RhoGEF". Experimental Biology, San Diego, CA

\section{PUBLICATIONS}

1. LaPointe, AT, Landers, V, Westcott, C, and Sokoloski, KJ.: Production of noncapped genomic RNAs is critical to Sindbis virus disease and pathogenicity. mBio. 11:e02675-20, 2020.

2. LaPointe, AT, Moreno-Contreras, J, and Sokoloski, KJ.: Increasing the capping efficiency of the Sindbis virus nsP1 protein negatively affects viral infection. mBio. 9:e02342-18, 2018.

3. LaPointe, AT, Gebhart, NN, Meller, ME, Hardy, RW, and Sokoloski, $\mathrm{KJ}$.: The identification and characterization of Sindbis virus RNA:host protein interactions. Journal of Virology. 92:e02171-17, 2018.

4. Martin, JW, Cavagnini, KS, Brawley, DN, Berkley, CY, Smolski, WC, Garcia, RD, Towne, AL, Sims, JR, and Meigs, TE.: A Ga12-specific binding domain in AKAP-Lbc and p114RhoGEF. Journal of Molecular Signaling. 11:3, 2016. 